\title{
The Gambler's and Hot-Hand Fallacies: Theory and Applications
}

\author{
Matthew Rabin* \\ Department of Economics \\ University of California, Berkeley
}

\author{
Dimitri Vayanos ${ }^{\dagger}$ \\ London School of Economics \\ CEPR and NBER
}

January $5,2007^{\ddagger}$

\begin{abstract}
We develop a model of the gambler's fallacy - the mistaken belief that random sequences should exhibit systematic reversals. We show that an individual who holds this belief and observes a sequence of signals can exaggerate the magnitude of changes in an underlying state but underestimate their duration. When the state is constant, and so signals are i.i.d., the individual can predict that long streaks of similar signals will continue - a hot-hand fallacy. When signals are serially correlated, the individual typically under-reacts to short streaks, over-reacts to longer ones, and under-reacts to very long ones. We explore several applications, showing, for example, that investors may move assets too much in and out of mutual funds, and exaggerate the value of financial information and expertise.
\end{abstract}

*rabin@econ. berkeley.edu

$\dagger$ d.vayanos@lse.ac.uk

†This paper was circulated previously under the title "The Gambler’s and Hot-Hand Fallacies in a Dynamic-Inference Model." We thank Anat Admati, Markus Brunnermeier, Eddie Dekel, Peter DeMarzo, Michael Jansson, Leonid Kogan, Jon Lewellen, Anna Pavlova, Francisco Penaranda, Paul Pfleiderer, Jean Tirole, three anonymous referees, and seminar participants at LSE and MIT for useful comments. Jeff Holman provided excellent research assistance. Rabin acknowledges financial support from the NSF. 


\section{Introduction}

Many people fall under the spell of the "gambler's fallacy," expecting outcomes in random sequences to exhibit systematic reversals. When observing flips of a fair coin, for example, people believe that a streak of heads makes it more likely that the next flip will be a tail. The gambler's fallacy is commonly interpreted as deriving from a fallacious belief in the "law of small numbers" or "local representativeness," namely that a small sample should resemble closely the underlying population. For example, people seem to believe that heads and tails should balance even in small samples. On the other hand, people also sometimes predict that random sequences will exhibit excessive persistence rather than reversals. While several studies have shown the belief to be fallacious, basketball fans believe that players have "hot hands," being more likely than average to make the next shot when currently on a hot streak. ${ }^{1}$

At first blush, the hot-hand fallacy appears to directly contradict the gambler's fallacy because it involves belief in excessive persistence rather than reversals. Several researchers have, however, suggested that the two fallacies might be related, with the hot-hand fallacy arising as a consequence of the gambler's fallacy. ${ }^{2}$ Consider an investor who believes that the performance of a mutual fund is a combination of the manager's ability and luck. Convinced that luck should revert, the investor underestimates the likelihood that a manager of average ability will exhibit a streak of above- or below-average performances. Following good or bad streaks, therefore, the investor will over-infer that the current manager is above or below average, and so in turn will predict continuation of unusual performances.

This paper develops a model to examine the link between the gambler's fallacy and the hot-hand fallacy, as well as the broader implications of the fallacies for people's predictions and actions in economic and financial settings. In our model, an individual observes a sequence of signals that depend on an unobservable underlying state. We show that because

\footnotetext{
${ }^{1}$ The representativeness bias is perhaps the most commonly explored bias in judgment research. Section 2 reviews evidence on the gambler's fallacy, and a more extensive review can be found in Rabin (2002). For evidence on the hot-hand fallacy see, for example, Gilovich, Vallone, and Tversky (1985) and Tversky and Gilovich (1989a, 1989b). See also Camerer (1989) who shows that betting markets for basketball games exhibit a small hot-hand bias.

${ }^{2}$ See, for example, Camerer (1989) and Rabin (2002). The causal link between the gambler's fallacy and the hot-hand fallacy is a common intuition in psychology. Some suggestive evidence comes from an experiment by Edwards (1961) in which subjects observe a very long binary series and are given no information about the generating process. Subjects seem, by the evolution of their predictions over time, to come to believe in a hot hand. Since the actual generating process is i.i.d., this is suggestive that a source of the hot hand is the perception of too many long streaks.
} 
of the gambler's fallacy, the individual is prone to exaggerate the magnitude of changes in the state, but underestimate their duration. We characterize the individual's predictions following streaks of similar signals, and determine when a hot-hand fallacy can arise. We study two applications to investor behavior: the movement of assets in and out of mutual funds, and the investors' willingness to pay for financial information.

While providing extensive motivation and elaboration in Section 2, our full model is presented here. An individual observes a sequence of signals whose probability distribution depends on an underlying state. The signal $s_{t}$ in Period $t=1,2, \ldots$ is

$$
s_{t}=\theta_{t}+\epsilon_{t},
$$

where $\theta_{t}$ is the state and $\epsilon_{t}$ an $i . i . d$. normal shock with mean zero and variance $\sigma_{\epsilon}^{2}>0$. The state evolves according to the auto-regressive process

$$
\theta_{t}=\mu+\rho\left(\theta_{t-1}-\mu\right)+\eta_{t}
$$

where $\mu$ is the long-run mean, $\rho \in[0,1)$ the persistence parameter, and $\eta_{t}$ an i.i.d. normal shock with mean zero, variance $\sigma_{\eta}^{2}$, and independent of $\epsilon_{t}$. As an example that we shall return to often, consider a mutual fund run by a team of managers. We interpret the signal as the fund's return, the state as the managers' average ability, and the shock $\epsilon_{t}$ as the managers' luck. Assuming that the ability of any given manager is constant over time, we interpret $1-\rho$ as the rate of managerial turnover, and $\sigma_{\eta}^{2}$ as the dispersion in ability across managers.

We model the gambler's fallacy as the mistaken belief that the sequence $\left\{\epsilon_{t}\right\}_{t \geq 1}$ is not i.i.d., but rather exhibits reversals: according to the individual,

$$
\epsilon_{t}=\omega_{t}-\alpha_{\rho} \sum_{k=0}^{\infty} \delta_{\rho}^{k} \epsilon_{t-1-k}
$$

where the sequence $\left\{\omega_{t}\right\}_{t \geq 1}$ is i.i.d. normal with mean zero and variance $\sigma_{\omega}^{2}, \alpha_{\rho} \equiv \alpha \rho$, and $\delta_{\rho} \equiv \delta \rho$, for some $\alpha, \delta \in[0,1){ }^{3}$ Intuitively, consistent with the gambler's fallacy, the individual believes that high realizations of $\epsilon_{t^{\prime}}$ in Period $t^{\prime}<t$ make a low realization more likely in Period $t$. The parameter $\alpha_{\rho} \in[0,1)$ measures the strength of the effect, while

\footnotetext{
${ }^{3}$ We set $\epsilon_{t}=0$ for $t \leq 0$, so that all terms in the infinite sum are well defined.
} 
$\delta_{\rho} \in[0,1)$ measures the relative influence of realizations in the recent and more distant past. We assume that $\left(\alpha_{\rho}, \delta_{\rho}\right)$ are linear functions of $\rho$. Our motivation for doing so is our interpretation of $\rho$ combined with the notion that people expect a small sample to resemble closely the underlying population only when all elements in the sample are drawn from the same intrinsic distribution. This is the case, for example, for the sample of performances of a fund manager whose ability is constant over time. But we assume that people do not structure the world as demanding forms of mean reversion across distributions; a good performance by one manager, for example, does not make another manager due for a bad performance next month. If managers turn over frequently (i.e., $\rho$ is small) therefore, the gambler's fallacy has a small effect. ${ }^{4}$ We develop and motivate these foundations in greater detail in Section 2. For expositional ease, from now on we follow the convention in the literature of referring to an individual subject to the gambler's fallacy $(\alpha>0)$ as "Freddy," and to a Bayesian $(\alpha=0)$ as "Tommy." 5

In Section 3 we examine how Freddy uses the sequence of past signals to make inferences about the underlying parameters and to predict future signals. We assume that Freddy infers as a fully rational Bayesian and fully understands the structure of his environment, except for a mistaken and dogmatic belief that $\alpha>0$. From observing the signals, Freddy infers both the underlying state $\theta_{t}$ and the values of any parameters of his model (i.e., $\sigma_{\eta}^{2}, \rho, \sigma_{\omega}^{2}, \mu$ ) about which he is uncertain.

When Freddy is certain about all model parameters, his inference about unobservable variables can be treated using standard tools of recursive (Kalman) filtering, where the gambler's fallacy essentially expands the state vector to include not only the state $\theta_{t}$ but also a statistic of past luck realizations. When Freddy is uncertain about parameters, recursive filtering can be used to evaluate the likelihood of signals conditional on parameters. An appropriate version of the law of large numbers then implies that after observing many signals, Freddy converges with probability one to parameter values that maximize a limit likelihood. While the maximum likelihood when $\alpha=0$ leads Tommy to limit posteriors corresponding to the true parameter values, Freddy's abiding belief that $\alpha>0$ leads him generally to false limit posteriors. Identifying when and how these limit beliefs are wrong is the crux of our analysis.

\footnotetext{
${ }^{4}$ An earlier version of this paper (Rabin and Vayanos (2005)) assumes that $\left(\alpha_{\rho}, \delta_{\rho}\right)$ are independent of $\rho$, and derives results of a similar flavor as long as $\delta_{\rho}<\rho$.

${ }^{5}$ Freddy made his first appearance in Rabin (2002)'s model of the law of small numbers. We relate our work to Rabin in Section 2.
} 
In Section 4 we consider the case where signals are i.i.d. This case can arise either because $\sigma_{\eta}^{2}=0$ or $\rho=0$, e.g., there is either no variance in managerial ability or all managers are replaced by new ones in each period. If Freddy is initially uncertain about the values of all parameters, then irrespective of whether $\sigma_{\eta}^{2}=0$ or $\rho=0$, he converges to the belief that $\rho=0$. Under this belief he predicts the signals correctly as i.i.d., despite the gambler's fallacy. The intuition is that he views each signal as drawn by a new distribution, e.g., new managers run the fund in each period. Therefore, his belief that any given manager's performance should average out over multiple periods has no effect.

We next assume that Freddy has prior knowledge that $\rho>0$, e.g., he is aware that managers stay in the fund for more than one period. This case is consistent with signals being i.i.d. as long as $\sigma_{\eta}^{2}=0$. Ironically, Freddy is worse off with prior knowledge because he cannot converge to the belief $\rho=0$, which, while incorrect, enables him to predict the signals correctly. Instead, he converges to the smallest value of $\rho$ to which he gives positive probability. He also converges to a positive value of $\sigma_{\eta}^{2}$, believing falsely that managers differ in ability, so that (given turnover) there is variation over time in average ability. This belief helps him explain the incidence of streaks despite the gambler's fallacy: a streak of high returns, for example, can be readily explained through the belief that good managers might have joined the fund recently. Of course, Freddy thinks the streak might also have been due to luck, and expects a reversal. We show that the expectation of a reversal dominates for short streaks, but because reversals that do not happen make Freddy more confident the managers have improved, he expects long streaks to continue.

In Section 5 we consider the case where signals are serially correlated. As in the i.i.d. case, Freddy underestimates $\rho$ and overestimates $\sigma_{\eta}^{2}$. He does not converge, however, all the way to $\rho=0$ because he must account for the signals' serial correlation. Because he views shocks to the state as overly large in magnitude (large $\sigma_{\eta}^{2}$ ), he treats signals as very informative, and tends to over-react to streaks. For very long streaks, however, there is under-reaction because Freddy's underestimation of $\rho$ means that he views the information learned from the signals as overly short-lived. Under-reaction also tends to occur following short streaks because of the basic gambler's fallacy intuition - Freddy expects luck to reverse.

In summary, Sections 4 and 5 confirm the oft-conjectured link from the gambler's to the hot-hand fallacy and generate additional predictions. We show that the hot-hand fallacy can arise in i.i.d. settings if individuals attribute non-zero prior probability that the state is time-varying $\left(\sigma_{\eta}^{2}>0\right)$. Moreover, if the fallacy arises, it does so following long streaks. 
The hot-hand fallacy always arises in non-i.i.d. settings, in the form of over-reaction relative to the rational benchmark. Over-reaction occurs following long streaks, but there is underreaction for very long streaks and possibly for short ones as well.

We conclude this paper in Section 6 by exploring the implications of our model for financial decisions. We interpret signals as financial returns, and assume that Freddy allocates wealth between a risky and a riskless asset under CARA preferences. A first application concerns Freddy's trading activity. When returns are i.i.d. and Freddy has prior knowledge that $\rho$ is bounded away from zero, he ends up believing in predictability. This false belief can lead Freddy to trade, unlike Tommy who recognizes that there is no predictability. More surprisingly, over-trading can also occur when there is true predictability and Freddy has no prior knowledge. The intuition is that Freddy views signals as overly informative about the state but treats the information as short-lived. Thus, his response to news is to make a large trade and reverse it shortly afterwards. These results could be relevant for understanding the large trading volume in actual markets and the large flows in and out of mutual funds.

A second application concerns the willingness to pay for financial information. Because Freddy can end up believing in predictability even in i.i.d. settings, he can overpay for information on past returns. He can also exaggerate the value of financial experts if he believes that the experts' advantage is to observe past returns. This could help explain why people invest in actively-managed funds in spite of the evidence that these funds do not outperform their market benchmarks. In Section 6 we argue that our model could also speak to other finance puzzles such as the equity-premium puzzle and the presence of momentum and reversals in stock returns.

\section{The Model}

Our model is fully described by Eqs. (1) to (3) presented in the Introduction. In this section we motivate the model by drawing the connection with the experimental evidence on the gambler's fallacy. A difficulty with using this evidence is that most experiments concern sequences that are both binary and i.i.d., such as coin flips. Our goal, by contrast, is to explore the implications of the gambler's fallacy in richer settings. Indeed, since our prediction is that Freddy will always come to believe the world is non-i.i.d., a theory of his beliefs in this perceived setting is essential. The experimental evidence gives little direct guidance as 
to how the gambler's fallacy would manifest itself in non-binary, non-i.i.d. settings. In this section, however, we make the case that our model represents a natural extrapolation of the "logic" underlying the behavior of the experimental subjects to richer settings. Of course, any such extrapolation has an element of arbitrariness to it. But, if nothing else, our specification of the gambler's fallacy in the new settings can be viewed as a working hypothesis about the broader empirical nature of the phenomenon that both highlights features of the phenomenon that seem to matter and generates testable predictions for experimental and field research.

Experiments documenting the gambler's fallacy are mainly of three types: production tasks, where subjects are asked to produce sequences that look to them like random sequences of coin flips, recognition tasks, where subjects are asked to identify which sequences look like coin flips, and prediction tasks, where subjects are asked to predict the next outcome in coin-flip sequences. In all types of experiments, the typical subject identifies a switching (i.e., reversal) rate greater than $50 \%$ as indicative of random coin flips. ${ }^{6}$ The most carefully reported data for our purposes comes from the production-task study of Rapoport and Budescu (1997). Using their Table 7, we estimate in Table 1 below the subjects' assessed probability that the next flip of a coin will be heads given the last three flips. ${ }^{7}$

According to Table 1, the average effect of changing the most recent flip from heads $(\mathrm{H})$ to tails $(\mathrm{T})$ is to raise the probability that the next flip will be $\mathrm{H}$ from $40.2 \%$ (= $\left.\frac{30.1 \%+38.1 \%+41.1 \%+51.5 \%}{4}\right)$ to $59.8 \%$, i.e., an increase of $19.6 \%$. This corresponds well to the

\footnotetext{
${ }^{6}$ See Bar-Hillel and Wagenaar (1991) for a review of the literature, and Rapoport and Budescu $(1992,1997)$ and Budescu and Rapoport (1994) for more recent studies. The experimental evidence has some shortcomings. For example, most prediction-task studies report the fraction of subjects predicting a switch but not the subjects' assessed probability of a switch. Thus, it could be that the vast majority of subjects predict a switch, and yet their assessed probability is only marginally larger than $50 \%$. Even worse, the probability could be exactly $50 \%$, since under that probability subjects are indifferent as to their prediction.

Some prediction-task studies attempt to measure assessed probabilities more accurately. For example, Gold and Hester (1987) find evidence in support of the gambler's fallacy in settings where subjects are given a choice between a sure payoff and a random payoff contingent on a specific coin outcome. Supporting evidence also comes from settings outside the laboratory. For example, Clotfelter and Cook (1993) and Terrell (1994) study pari-mutuel lotteries, where the winnings from a number are shared among all people betting on that number. They find that people avoid systematically to bet on numbers that won recently. This is a strict mistake because the numbers with the fewest bets are those with the largest expected winnings.

${ }^{7}$ Rapoport and Budescu report occurrences of short sequences of heads $(\mathrm{H})$ and tails $(\mathrm{T})$ within the larger sequences (of 150 elements) produced by the subjects. To translate these occurrences into conditional probabilities, we use relative frequencies: for example, we estimate the probability of $\mathrm{H}$ conditional on the sequence HHH as the ratio of occurrences of HHHH to the sum of occurrences of HHHH and HHHT. This represents the fraction of the time that subjects chose to repeat $\mathrm{H}$ after $\mathrm{HHH}$. We believe that this fraction is a good measure of the subjects' assessed probability: for example, a subject believing that HHH should be followed by $\mathrm{H}$ with $40 \%$ probability could be choosing $\mathrm{H}$ after $\mathrm{HHH} 40 \%$ of the time when constructing a random sequence.
} 


\begin{tabular}{|ccc|c|}
\hline 3rd-to-last & 2nd-to-last & Very last & Prob. next will be H (\%) \\
\hline \hline $\mathrm{H}$ & $\mathrm{H}$ & $\mathrm{H}$ & 30.1 \\
\hline $\mathrm{T}$ & $\mathrm{H}$ & $\mathrm{H}$ & 38.1 \\
\hline $\mathrm{H}$ & $\mathrm{T}$ & $\mathrm{H}$ & 41.1 \\
\hline $\mathrm{H}$ & $\mathrm{H}$ & $\mathrm{T}$ & 48.5 \\
\hline $\mathrm{H}$ & $\mathrm{T}$ & $\mathrm{T}$ & 61.9 \\
\hline $\mathrm{T}$ & $\mathrm{H}$ & $\mathrm{T}$ & 58.9 \\
\hline $\mathrm{T}$ & $\mathrm{T}$ & $\mathrm{H}$ & 51.5 \\
\hline $\mathrm{T}$ & $\mathrm{T}$ & $\mathrm{T}$ & 69.9 \\
\hline
\end{tabular}

Table 1: Assessed probability that the next flip of a coin will be heads $(\mathrm{H})$ given the last three flips being heads or tails (T). Based on Rapoport and Budescu (1997), Table 7, p. 613.

general stylized fact in the literature that subjects tend to view randomness in coin-flip sequences as corresponding to a switching rate of $60 \%$ rather than $50 \%$. Table 1 also shows that the effect of the gambler's fallacy is not limited to the most recent flip. For example, the average effect of changing the second most recent flip from $\mathrm{H}$ to $\mathrm{T}$ is to raise the probability of $\mathrm{H}$ from $43.9 \%$ to $56.1 \%$, i.e., an increase of $12.2 \%$. The average effect of changing the third most recent flip from $\mathrm{H}$ to $\mathrm{T}$ is to raise the probability of $\mathrm{H}$ from $45.4 \%$ to $54.6 \%$, i.e., an increase of $9.2 \%$.

How would a believer in the gambler's fallacy, exhibiting behavior such as in Table 1, form predictions in non-binary, non-i.i.d. settings? Our extrapolation approach consists in viewing the richer settings as combinations of coins. We first consider a setting that is nonbinary but i.i.d. Suppose that the signal Freddy is observing in each period is the sum of a large number of independent coin flips, where we set $\mathrm{H}=1$ and $\mathrm{T}=-1$. For example, with 100 coins, Freddy is observing a signal between 100 and -100 , and a signal of 10 means that 55 coins came $\mathrm{H}$ and 45 came T. Suppose that Freddy applies his gambler's fallacy reasoning to each of the coins, and his beliefs are as in Table 1. Then, after a signal of 10, he assumes that the $55 \mathrm{H}$ coins have probability $40.2 \%$ to come $\mathrm{H}$ again, while the $45 \mathrm{~T}$ coins have probability $59.8 \%$ to switch to $\mathrm{H}$. Thus, he expects on average $40.2 \% \times 55+59.8 \% \times 45=49.02$ coins to come $\mathrm{H}$, and this translates to an expectation of $49.02-(100-49.02)=-1.96$ for the next signal.

The "multiple-coin" story shares many of our model's key features. To explain why, we specialize the model to i.i.d. signals, taking the state $\theta_{t}$ to be known and constant over time. We generate a constant state by setting $\rho=1$ and $\sigma_{\eta}^{2}=0$ in (2). For simplicity, we also 
normalize the constant value of the state to zero. For $\rho=1,(3)$ becomes

$$
\epsilon_{t}=\omega_{t}-\alpha \sum_{k=0}^{\infty} \delta^{k} \epsilon_{t-1-k} .
$$

When the state is equal to zero, (1) becomes $s_{t}=\epsilon_{t}$. Substituting into (4) and taking expectations conditional on Period $t-1$, we find

$$
E_{t-1}\left(s_{t}\right)=-\alpha \sum_{k=0}^{\infty} \delta^{k} s_{t-1-k} .
$$

Comparing (5) with the predictions of the multiple-coin story, we can calibrate the parameters $(\alpha, \delta)$ and test our model specification. Suppose that $s_{t-1}=10$ and that signals in prior periods are equal to their average of zero. Eq. (5) then implies that $E_{t-1}\left(s_{t}\right)=-10 \alpha$. According to the multiple-coin story $E_{t-1}\left(s_{t}\right)$ should be -1.96 , which yields $\alpha=-0.196$. To calibrate $\delta$, we repeat the exercise for $s_{t-2}$ and $s_{t-3}$. Using the data in Table 1 , we find $\alpha \delta=-0.122$ and $\alpha \delta^{2}=-0.096$. Thus, the decay in influence between the most and second most recent signal is $0.122 / 0.196=0.62$, while the decay between the second and third most recent signal is $0.092 / 0.122=0.75$. The two rates are not identical as our model assumes, but are quite close. Thus, our geometric-decay specification seems reasonable, and we can take $\alpha=0.2$ and $\delta=0.7$ as a plausible calibration. With the obvious caution that this is based on the limited evidence of one experiment, we use these values as part of a more general calibration exercise in Section 6.4.

Several other features of our specification deserve comment. One is normality: since $\omega_{t}$ is normal, (4) implies that the distribution of $s_{t}=\epsilon_{t}$ conditional on Period $t-1$ is normal. The multiple-coin story also generates approximate normality if we take the number of coins to be large. A second feature is linearity: if we double $s_{t-1}$ in (5), holding other signals to zero, then $E_{t-1}\left(s_{t}\right)$ doubles. The multiple-coin story shares this feature: a signal of 20 means that 60 coins came $\mathrm{H}$ and 40 came $\mathrm{T}$, and this doubles the expectation of the next signal. A third feature is additivity: according to (5), the effect of each signal on $E_{t-1}\left(s_{t}\right)$ is independent of the other signals. Table 1 generates some support for additivity. For example, changing the most recent flip from $\mathrm{H}$ to $\mathrm{T}$ increases the probability of $\mathrm{H}$ by $20.8 \%$ when the second and third most recent flips are identical (HH or TT) and by $18.4 \%$ when they differ (HT or TH). Thus, in this experiment the effect of the most recent flip depends only weakly on prior flips.

We next extend our approach to non-i.i.d. settings. Suppose that the signal Freddy 
is observing in each period is the sum of a large number of independent coin flips, but where coins differ in their probabilities of $\mathrm{H}$ and $\mathrm{T}$, and are replaced over time randomly by new coins. Signals are thus serially correlated: they tend to be high at times where the replacement process brings many new coins biased towards $H$, and vice-versa. If Freddy applies his gambler's fallacy reasoning to each individual coin, then this will generate a gambler's fallacy for the signals. The strength of the latter fallacy, however, depends on the rate at which coins are replaced. If, for example, Freddy believes that replacement is frequent, then his incorrect belief that the outcomes of a given coin should average out over time has little effect.

To relate the above story to our model, we recast it in a setting with normal distributions. Consider a mutual fund that consists of a continuum with mass one of managers, and suppose that a random fraction $1-\rho$ of managers are replaced by new ones in each period. Suppose that the fund's return $s_{t}$ is an average of returns attributable to each manager, and a manager's return is the sum of ability and luck, both normally distributed. Ability is constant over time for a given manager, while luck is i.i.d. Thus, a manager's returns are i.i.d. conditional on ability, and the manager can be viewed as a "coin" with the probability of $\mathrm{H}$ and $\mathrm{T}$ corresponding to ability. To ensure that aggregate variables are stochastic despite the continuum assumption, we assume that ability and luck are identical within the cohort of managers who enter the fund in a given period. ${ }^{8}$

We next show that if Freddy applies his gambler's fallacy reasoning to each manager, per our specification (4) for i.i.d. settings, then this generates a gambler's fallacy for fund returns, per our specification (3) for non-i.i.d. settings. Defining $\epsilon_{t, t^{\prime}}$ as the luck in Period $t$ of the cohort entering in Period $t^{\prime} \leq t$, we can write (4) for a manager in that cohort as

$$
\epsilon_{t, t^{\prime}}=\omega_{t, t^{\prime}}-\alpha \sum_{k=0}^{\infty} \delta^{k} \epsilon_{t-1-k, t^{\prime}}
$$

where $\left\{\omega_{t, t^{\prime}}\right\}_{t \geq t^{\prime} \geq 0}$ is an i.i.d. sequence, and $\epsilon_{t^{\prime \prime}, t^{\prime}} \equiv 0$ for $t^{\prime \prime}<t^{\prime}$. To aggregate (6) for the fund, we note that in Period $t$ the average luck $\epsilon_{t}$ of all managers is

$$
\epsilon_{t}=(1-\rho) \sum_{t^{\prime} \leq t} \rho^{t-t^{\prime}} \epsilon_{t, t^{\prime}}
$$

\footnotetext{
${ }^{8}$ The intuition behind the example would be the same, but more plausible, with a single manager in each period who is replaced by a new one with Poisson probability $1-\rho$. We assume a continuum because this preserves normality. The assumption that all managers in a cohort have the same ability and luck can be motivated in reference to the single-manager setting.
} 
since $(1-\rho) \rho^{t-t^{\prime}}$ managers from the cohort entering in Period $t^{\prime}$ are still in the fund. Combining (6) and (7), we find

$$
\epsilon_{t}=\omega_{t}-\alpha_{\rho} \sum_{k=0}^{\infty} \delta_{\rho}^{k} \epsilon_{t-1-k}
$$

for $\alpha_{\rho} \equiv \alpha \rho, \delta_{\rho} \equiv \delta \rho$, and the $i . i . d$. sequence $\omega_{t} \equiv(1-\rho) \sum_{t^{\prime} \leq t} \rho^{t-t^{\prime}} \omega_{t, t^{\prime}}$. Eq. (8) coincides with (3), our specification of the gambler's fallacy in non-i.i.d. settings. The difference between (8) and its i.i.d. counterpart (4) is that the parameters $(\alpha, \delta)$ of the gambler's fallacy are multiplied by $\rho$. Thus, the fallacy is weaker the larger managerial turnover is. Intuitively, with large turnover, Freddy's belief that a given manager's performance should average out over multiple periods has little effect. ${ }^{9}$

The model closest to ours, and from which we build, is Rabin's (2002) model of the law of small numbers. In Rabin, the signal is the color of a ball drawn from an urn. Balls are drawn with replacement, but Freddy believes that the urn is replenished only every odd period. Thus, he overestimates the probability that the ball drawn in an even period is of a different color than the one drawn in the previous period.

The model employed here provides a broader framework with substantial additional structure, and addresses a wider range of questions. Besides eliminating the artificial distinction between odd and even periods, which Rabin introduces to prevent the urn from running out of balls, we assume continuous rather than binary signals, and move beyond i.i.d. settings because we allow the state (urn type in Rabin) to vary over time. This makes our model suitable for analyzing the hot-hand fallacy, which involves precisely a belief in the state's time-variation. ${ }^{10}$ Within our model we can also examine how frequently Freddy believes that the state is changing, and many of our results are based on his overestimation of that frequency. Finally, because of the time-varying state, learning in our model continues to occur in a stochastic steady state, while learning eventually ceases in a constant-state model.

Because of the steady state and the normal-linear structure, our model is more tractable and suitable for applications. For example, we can characterize fully Freddy's predictions

\footnotetext{
${ }^{9}$ Our specification of the gambler's fallacy becomes ambiguous in the special case where Freddy believes that while managers turn over $(\rho<1)$, they are all of the same ability $\left(\sigma_{\eta}^{2}=0\right)$. In that case, he views the returns of all managers as generated by a common distribution. Therefore, his fallacious belief on small samples can apply equally plausibly to the common distribution as to the distribution of a single manager's returns. In other words, (8) is equally plausible for $(\alpha, \delta)$ as for $\left(\alpha_{\rho}, \delta_{\rho}\right)$, i.e., $\rho$ can be replaced by one. In what follows we ignore this ambiguity since in almost all cases we consider Freddy entertains the possibility that $\sigma_{\eta}^{2}>0$, thus viewing different managers as potentially different distributions.

${ }^{10}$ Rabin shows a static cousin of the hot-hand fallacy called fictitious variation: Freddy overestimates the cross-sectional dispersion in urn types.
} 
after streaks of signals, while Rabin can do so (with numerical examples) only for short streaks. The Finance applications in Section 6 illustrate further our model's tractability and applicability. We show that quantities such as trading volume and value of information can be computed easily, and we establish several novel results on how the gambler's fallacy influences these quantities.

\section{Inference - General Results}

In this section we formulate Freddy's inference problem, and establish some general results that serve as the basis for the more specific results of Sections 4-6. The inference problem consists in using the signals to learn about the underlying state $\theta_{t}$ and possibly about the parameters of the model. Freddy's model is characterized by the variance $\sigma_{\eta}^{2}$ of the shocks to the state, the persistence $\rho$, the variance $\sigma_{\omega}^{2}$ of the shocks affecting the signal noise, the long-run mean $\mu$, and the parameters $(\alpha, \delta)$ of the gambler's fallacy. We assume that Freddy does not question his belief in the gambler's fallacy, i.e., has a dogmatic point prior on $(\alpha, \delta)$. He can, however, learn about the other parameters. From now on, we reserve the notation $\left(\sigma_{\eta}^{2}, \rho, \sigma_{\omega}^{2}, \mu\right)$ for the true parameter values, and denote generic values by $\left(\tilde{\sigma}_{\eta}^{2}, \tilde{\rho}, \tilde{\sigma}_{\omega}^{2}, \tilde{\mu}\right)$. Thus, Freddy can learn about the parameter vector $\tilde{p} \equiv\left(\tilde{\sigma}_{\eta}^{2}, \tilde{\rho}, \tilde{\sigma}_{\omega}^{2}, \tilde{\mu}\right)$.

\subsection{No Parameter Uncertainty}

We start with the case where Freddy is certain that the parameter vector takes a specific value $\tilde{p}$. This case is relatively simple and serves as an input for the parameter-uncertainty case. Freddy's inference problem can be formulated as one of recursive (Kalman) filtering. Recursive filtering is a technique for solving inference problems where (i) inference concerns a "state vector" evolving according to a stochastic process, (ii) a noisy signal of the state vector is observed in each period, (iii) the stochastic structure is linear and normal. ${ }^{11}$

To formulate the recursive-filtering problem, we must define the state vector, the equation according to which the state vector evolves, and the equation linking the state vector to the signal. The state vector must include not only the state $\theta_{t}$, but also some measure of the past

\footnotetext{
${ }^{11}$ For textbooks on recursive filtering see, for example, Anderson and Moore (1979) and Balakrishnan (1987). We are using the somewhat cumbersome term "state vector" because we are reserving the term "state" for $\theta_{t}$, and the two concepts differ in our model.
} 
realizations of luck since according to Freddy luck reverses predictably. It turns out that all past luck realizations can be condensed into an one-dimensional statistic. This statistic can be appended to the state $\theta_{t}$, and therefore, recursive filtering can be used even in the presence of the gambler's fallacy. We define the state vector as

$$
x_{t} \equiv\left[\theta_{t}-\tilde{\mu}, \epsilon_{t}^{\delta}\right]^{\prime}
$$

where the statistic of past luck realizations is

$$
\epsilon_{t}^{\delta} \equiv \sum_{k=0}^{\infty} \delta_{\tilde{\rho}}^{k} \epsilon_{t-k}
$$

and $v^{\prime}$ denotes the transpose of the vector $v$. Eqs. (2) and (3) imply that the state vector evolves according to

$$
x_{t}=\tilde{A} x_{t-1}+w_{t}
$$

where

$$
\tilde{A} \equiv\left[\begin{array}{cc}
\tilde{\rho} & 0 \\
0 & \delta_{\tilde{\rho}}-\alpha_{\tilde{\rho}}
\end{array}\right]
$$

and

$$
w_{t} \equiv\left[\eta_{t}, \omega_{t}\right]^{\prime}
$$

Eqs. (1)-(3) imply that the signal is related to the state vector through

$$
s_{t}=\tilde{\mu}+\tilde{C} x_{t-1}+v_{t}
$$

where

$$
\tilde{C} \equiv\left[\tilde{\rho},-\alpha_{\tilde{\rho}}\right]
$$

and $v_{t} \equiv \eta_{t}+\omega_{t}$. To start the recursion, we must specify Freddy's prior beliefs for the initial state $x_{0}$. We denote the mean and variance of $\theta_{0}$ by $\bar{\theta}_{0}$ and $\sigma_{\theta, 0}^{2}$, respectively. Since $\epsilon_{t}=0$ for $t \leq 0$, the mean and variance of $\epsilon_{0}^{\delta}$ are zero. Proposition 1 determines Freddy's beliefs about the state in Period $t$, conditional on the history of signals $\mathcal{H}_{t} \equiv\left\{s_{t^{\prime}}\right\}_{t^{\prime}=1, . ., t}$ up to that period.

Proposition 1 Conditional on $\mathcal{H}_{t}, x_{t}$ is normal with mean $\bar{x}_{t}(\tilde{p})$ given recursively by

$$
\bar{x}_{t}(\tilde{p})=\tilde{A} \bar{x}_{t-1}(\tilde{p})+\tilde{G}_{t}\left[s_{t}-\tilde{\mu}-\tilde{C} \bar{x}_{t-1}(\tilde{p})\right], \quad \bar{x}_{0}(\tilde{p})=\left[\bar{\theta}_{0}-\tilde{\mu}, 0\right]^{\prime},
$$


and covariance matrix $\tilde{\Sigma}_{t}$ given recursively by

$$
\tilde{\Sigma}{ }_{t}=\tilde{A} \tilde{\Sigma}_{t-1} \tilde{A}^{\prime}-\left[\tilde{C} \tilde{\Sigma}_{t-1} \tilde{C}^{\prime}+\tilde{V}\right] \tilde{G}_{t} \tilde{G}_{t}^{\prime}+\tilde{W}, \quad \tilde{\Sigma}_{0}=\left[\begin{array}{cc}
\sigma_{\theta, 0}^{2} & 0 \\
0 & 0
\end{array}\right]
$$

where

$$
\tilde{G}_{t} \equiv \frac{1}{\tilde{C} \tilde{\Sigma}_{t-1} \tilde{C}^{\prime}+\tilde{V}}\left[\tilde{A} \tilde{\Sigma}_{t-1} \tilde{C}^{\prime}+\tilde{U}\right]
$$

$\tilde{V} \equiv \tilde{E}\left(v_{t}^{2}\right), \tilde{W} \equiv \tilde{E}\left(w_{t} w_{t}^{\prime}\right), \tilde{U} \equiv \tilde{E}\left(v_{t} w_{t}\right)$, and $\tilde{E}$ is Freddy's expectation operator.

Freddy's conditional expectation evolves according to (11). This is simply a regression equation: the state vector in Period $t$ is regressed on that period's signal, conditional on the history up to Period $t-1$. The regression coefficient $\tilde{G}_{t}$ depends on Freddy's conditional variance of the state $\tilde{\Sigma}_{t-1}$. Proposition 2 shows that when $t$ goes to $\infty$, this variance converges to a limit that is independent of the initial value $\tilde{\Sigma}_{0}$.

Proposition $2 \operatorname{Lim}_{t \rightarrow \infty} \tilde{\Sigma}_{t}=\tilde{\Sigma}$, where $\tilde{\Sigma}$ is the unique solution in the set of positive matrices of

$$
\tilde{\Sigma}=\tilde{A} \tilde{\Sigma} \tilde{A}^{\prime}-\frac{1}{\tilde{C} \tilde{\Sigma} \tilde{C}^{\prime}+\tilde{V}}\left[\tilde{A} \tilde{\Sigma} \tilde{C}^{\prime}+\tilde{U}\right]\left[\tilde{A} \tilde{\Sigma} \tilde{C}^{\prime}+\tilde{U}\right]^{\prime}+\tilde{W}
$$

Proposition 2 implies that there is convergence to a steady state where the conditional variance $\tilde{\Sigma}_{t}$ is equal to the constant $\tilde{\Sigma}$, the regression coefficient $\tilde{G}_{t}$ is equal to the constant

$$
\tilde{G} \equiv \frac{1}{\tilde{C} \tilde{\Sigma} \tilde{C}^{\prime}+\tilde{V}}\left[\tilde{A} \tilde{\Sigma} \tilde{C}^{\prime}+\tilde{U}\right]
$$

and the conditional expectation of the state vector $x_{t}$ evolves according to a linear equation with constant coefficients. This steady state plays an important role in our analysis: it is also the limit in the case of parameter uncertainty because Freddy eventually becomes certain about the parameter values.

\subsection{Parameter Uncertainty}

We next allow Freddy to be uncertain about the parameters of his model. Parameter uncertainty is a natural assumption in many settings. For example, Freddy might be uncertain about the extent to which fund managers differ in ability $\left(\sigma_{\eta}^{2}\right)$ or turn over $(\rho)$. 
Because parameter uncertainty eliminates the normality that is necessary for recursive filtering, Freddy's inference problem threatens to be less tractable. Recursive filtering can, however, be used as part of a two-stage procedure. In a first stage, we fix each model parameter to a given value and compute the likelihood of a history of signals conditional on these values. Because the conditional probability distribution is normal, the likelihood can be computed using the recursive-filtering formulas of Section 3.1. In a second stage, we combine the likelihood with Freddy's prior beliefs, through Bayes' law, and determine Freddy's posteriors on the parameters. We show, in particular, that Freddy's posteriors in the limit when $t$ goes to $\infty$ can be derived by maximizing a limit likelihood over all possible parameter values.

We describe Freddy's prior beliefs over parameter vectors by a probability measure $\pi_{0}$ and denote by $P$ the closed support of $\pi_{0} \cdot{ }^{12}$ As we show below, $\pi_{0}$ affects Freddy's limit posteriors only through $P$.

The likelihood function $L_{t}\left(\mathcal{H}_{t} \mid \tilde{p}\right)$ associated to a parameter vector $\tilde{p}$ and history $\mathcal{H}_{t}=$ $\left\{s_{t^{\prime}}\right\}_{t^{\prime}=1, . ., t}$ is the probability density of observing the signals conditional on $\tilde{p}$. From Bayes' law, this density is

$$
L_{t}\left(\mathcal{H}_{t} \mid \tilde{p}\right)=L_{t}\left(s_{1} \cdots s_{t} \mid \tilde{p}\right)=\prod_{t^{\prime}=1}^{t} \ell_{t^{\prime}}\left(s_{t^{\prime}} \mid s_{1} \cdots s_{t^{\prime}-1}, \tilde{p}\right)=\prod_{t^{\prime}=1}^{t} \ell_{t^{\prime}}\left(s_{t^{\prime}} \mid \mathcal{H}_{t^{\prime}-1}, \tilde{p}\right)
$$

where $\ell_{t}\left(s_{t} \mid \mathcal{H}_{t-1}, \tilde{p}\right)$ denotes the density of $s_{t}$ conditional on $\tilde{p}$ and $\mathcal{H}_{t-1}$. The latter density can be computed using the recursive-filtering formulas of Section 3.1. Indeed, Proposition 1 shows that conditional on $\tilde{p}$ and $\mathcal{H}_{t-1}, x_{t-1}$ is normal. Since $s_{t}$ is a linear function of $x_{t-1}$, it is also normal with a mean and variance that we denote by $\bar{s}_{t}(\tilde{p})$ and $\sigma_{s, t}^{2}(\tilde{p})$, respectively. Thus:

$$
\ell_{t}\left(s_{t} \mid \mathcal{H}_{t-1}, \tilde{p}\right)=\frac{1}{\sqrt{2 \pi \sigma_{s, t}^{2}(\tilde{p})}} \exp \left[-\frac{\left[s_{t}-\bar{s}_{t}(\tilde{p})\right]^{2}}{2 \sigma_{s, t}^{2}(\tilde{p})}\right]
$$

and

$$
L_{t}\left(\mathcal{H}_{t} \mid \tilde{p}\right)=\frac{1}{\sqrt{(2 \pi)^{t} \prod_{t^{\prime}=1}^{t} \sigma_{s, t^{\prime}}^{2}(\tilde{p})}} \exp \left[-\sum_{t^{\prime}=1}^{t} \frac{\left[s_{t^{\prime}}-\bar{s}_{t^{\prime}}(\tilde{p})\right]^{2}}{2 \sigma_{s, t^{\prime}}^{2}(\tilde{p})}\right]
$$

Freddy's posterior beliefs over parameter vectors can be derived from his prior beliefs and the likelihood through Bayes' law. To determine posteriors in the limit when $t$ goes to $\infty$, we

\footnotetext{
${ }^{12}$ The closed support of $\pi_{0}$ is the intersection of all closed sets $C$ such that $\pi_{0}(C)=1$. Any neighborhood $B$ of an element of the closed support satisfies $\pi_{0}(B)>0$. (Billingsley, 12.9, p.181)
} 
need to determine the asymptotic behavior of the likelihood function $L_{t}\left(\mathcal{H}_{t} \mid \tilde{p}\right)$. Intuitively, this behavior depends on how well Freddy can fit the data (i.e., the history of signals) using the model corresponding to $\tilde{p}$. To evaluate the fit of a model, we consider the true model according to which the data are generated. The true model is characterized by $\alpha=0$ and the true parameters $p \equiv\left(\sigma_{\eta}^{2}, \rho, \sigma_{\omega}^{2}, \mu\right)$. We denote by $\bar{s}_{t}$ and $\sigma_{s, t}^{2}$, respectively, the true mean and variance of $s_{t}$ conditional on $\mathcal{H}_{t-1}$, and by $E$ the true expectation operator.

\section{Theorem 1}

$$
\lim _{t \rightarrow \infty} \frac{\log L_{t}\left(\mathcal{H}_{t} \mid \tilde{p}\right)}{t}=-\frac{1}{2}\left[\log \left[2 \pi \sigma_{s}^{2}(\tilde{p})\right]+\frac{\sigma_{s}^{2}+e(\tilde{p})}{\sigma_{s}^{2}(\tilde{p})}\right] \equiv F(\tilde{p})
$$

almost surely, where

$$
\begin{aligned}
\sigma_{s}^{2}(\tilde{p}) & \equiv \lim _{t \rightarrow \infty} \sigma_{s, t}^{2}(\tilde{p}), \\
\sigma_{s}^{2} & \equiv \lim _{t \rightarrow \infty} \sigma_{s, t}^{2}, \\
e(\tilde{p}) & \equiv \lim _{t \rightarrow \infty} E\left[\bar{s}_{t}(\tilde{p})-\bar{s}_{t}\right]^{2} .
\end{aligned}
$$

Theorem 1 implies that the likelihood function is asymptotically equal to

$$
L_{t}\left(\mathcal{H}_{t} \mid \tilde{p}\right) \sim \exp [t F(\tilde{p})]
$$

thus growing exponentially at the rate $F(\tilde{p})$. Note that $F(\tilde{p})$ does not depend on the specific history $\mathcal{H}_{t}$ of signals, and is thus deterministic. That the likelihood function becomes deterministic for large $t$ follows from the law of large numbers, which is the main result that we need to prove the theorem. The appropriate large-numbers law in our setting is one applying to non-independent and non-identically distributed random variables. Non-independence is because the expected values $\bar{s}_{t}(\tilde{p})$ and $\bar{s}_{t}$ involve the entire history of past signals, and nonidentical distributions are because at any finite time we are not at the steady state.

The growth rate $F(\tilde{p})$ can be interpreted as the fit of the model corresponding to $\tilde{p}$. A natural conjecture is that when $t$ goes to $\infty$, Freddy gives weight only to values of $\tilde{p}$ that maximize $F(\tilde{p})$ over $P$. Lemma 1 confirms this conjecture under Assumption 1.

Assumption 1 The set $P$ takes the product form $P_{\sigma, \rho} \times P_{\mu}$, where $P_{\sigma, \rho} \subset \mathbb{R}^{3}$ is a set of values for $\left(\tilde{\sigma}_{\eta}^{2}, \tilde{\rho}, \tilde{\sigma}_{\omega}^{2}\right)$ and $P_{\mu} \subset \mathbb{R}$ a set of values for $\tilde{\mu}$. 
Lemma 1 Under Assumption 1, the set $m(P) \equiv \operatorname{argmax}_{\tilde{p} \in P} F(\tilde{p})$ is non-empty. When $t$ goes to $\infty$, and for almost all histories, the posterior measure $\pi_{t}$ converges weakly to a measure giving weight only to $m(P)$.

Lemma 2 characterizes the solution to the fit-maximization problem under Assumptions 1 and 2 .

Assumption 2 The set $P_{\sigma, \rho}$ satisfies the cone property

$$
\left(\tilde{\sigma}_{\eta}^{2}, \tilde{\rho}, \tilde{\sigma}_{\omega}^{2}\right) \in P_{\sigma, \rho} \Rightarrow\left(\lambda \tilde{\sigma}_{\eta}^{2}, \tilde{\rho}, \lambda \tilde{\sigma}_{\omega}^{2}\right) \in P_{\sigma, \rho}, \quad \forall \lambda>0
$$

Lemma 2 Under Assumptions 1 and $2, \tilde{p} \in m(P)$ if and only if

- $e(\tilde{p})=\min _{\tilde{p}^{\prime} \in P} e\left(\tilde{p}^{\prime}\right) \equiv e(P)$

- $\sigma_{s}^{2}(\tilde{p})=\sigma_{s}^{2}+e(\tilde{p})$.

The characterization of Lemma 2 is intuitive. The function $e(\tilde{p})$ is the expected squared difference between the conditional mean of $s_{t}$ that Freddy computes under $\tilde{p}$, and the true conditional mean. Thus, $e(\tilde{p})$ measures the error in Freddy's predictions relative to the true model, and a model maximizing the fit must minimize this error.

A model maximizing the fit must also generate the right measure of uncertainty about the future signals. Freddy's uncertainty under the model corresponding to $\tilde{p}$ is measured by $\sigma_{s}^{2}(\tilde{p})$, the conditional variance of $s_{t}$. This must equal to the true error in Freddy's predictions, which is the sum of two orthogonal components: the error $e(\tilde{p})$ relative to the true model, and the error in the true model's predictions, i.e., the true conditional variance $\sigma_{s}^{2}$.

The cone property ensures that in maximizing the fit, there is no conflict between minimizing $e(\tilde{p})$ and setting $\sigma_{s}^{2}(\tilde{p})=\sigma_{s}^{2}+e(\tilde{p})$. Indeed, $e(\tilde{p})$ depends on $\tilde{\sigma}_{\eta}^{2}$ and $\tilde{\sigma}_{\omega}^{2}$ only through their ratio because only the ratio affects the vector $G$ of regression coefficients. The cone property ensures that given any feasible ratio, we can scale $\tilde{\sigma}_{\eta}^{2}$ and $\tilde{\sigma}_{\omega}^{2}$ to make $\sigma_{s}^{2}(\tilde{p})$ equal to $\sigma_{s}^{2}+e(\tilde{p})$. The cone property is satisfied, in particular, when the set $P$ includes all parameter values: ${ }^{13}$

$$
P=P_{0} \equiv\left\{\left(\tilde{\sigma}_{\eta}^{2}, \tilde{\rho}, \tilde{\sigma}_{\omega}^{2}, \tilde{\mu}\right): \tilde{\sigma}_{\eta}^{2} \in \mathbb{R}^{+}, \tilde{\rho} \in[0,1], \tilde{\sigma}_{\omega}^{2} \in \mathbb{R}^{+}, \tilde{\mu} \in \mathbb{R}\right\} .
$$

\footnotetext{
${ }^{13}$ Although Freddy considers only $\tilde{\rho} \in[0,1), P_{0}$ includes $\tilde{\rho}=1$ because it is a closed set.
} 
Lemma 3 computes the error $e(\tilde{p})$. In both the lemma and subsequent analysis, we denote matrices corresponding to the true model by omitting the tilde. For example, the true-model counterpart of $\tilde{C} \equiv\left[\tilde{\rho},-\alpha_{\tilde{\rho}}\right]$ is $C \equiv[\rho, 0]$.

Lemma 3 The error $e(\tilde{p})$ is given by

$$
e(\tilde{p})=\sigma_{s}^{2} \sum_{k=1}^{\infty}\left(\tilde{N}_{k}-N_{k}\right)^{2}+\left(N^{\mu}\right)^{2}(\tilde{\mu}-\mu)^{2}
$$

where

$$
\begin{aligned}
\tilde{N}_{k} & \equiv \tilde{C}(\tilde{A}-\tilde{G} \tilde{C})^{k-1} \tilde{G}+\sum_{k^{\prime}=1}^{k-1} \tilde{C}(\tilde{A}-\tilde{G} \tilde{C})^{k-1-k^{\prime}} \tilde{G} C A^{k^{\prime}-1} G, \\
N_{k} & \equiv C A^{k-1} G \\
N^{\mu} & \equiv 1-\tilde{C} \sum_{k=1}^{\infty}(\tilde{A}-\tilde{G} \tilde{C})^{k-1} \tilde{G} .
\end{aligned}
$$

The terms $\tilde{N}_{k}$ and $N_{k}$ can be given an intuitive interpretation. Suppose that we are in steady state (i.e., a large number of periods have elapsed) and set $\zeta_{t} \equiv s_{t}-\bar{s}_{t}$. The shock $\zeta_{t}$ represents the "surprise" in Period $t$, i.e., the difference between the signal $s_{t}$ and its conditional mean $\bar{s}_{t}$ under the true model. The mean $\bar{s}_{t}$ is a linear function of the history $\left\{\zeta_{t^{\prime}}\right\}_{t^{\prime} \leq t-1}$ of past shocks, and $N_{k}$ is the impact of $\zeta_{t-k}$, i.e.,

$$
N_{k}=\frac{\partial \bar{s}_{t}}{\partial \zeta_{t-k}}=\frac{\partial E_{t-1}\left(s_{t}\right)}{\partial \zeta_{t-k}}
$$

The term $\tilde{N}_{k}$ is the counterpart of $N_{k}$ under Freddy's model, i.e.,

$$
\tilde{N}_{k}=\frac{\partial \bar{s}_{t}(\tilde{p})}{\partial \zeta_{t-k}}=\frac{\partial \tilde{E}_{t-1}\left(s_{t}\right)}{\partial \zeta_{t-k}}
$$

If $\tilde{N}_{k} \neq N_{k}$, then the shock $\zeta_{t-k}$ affects Freddy's mean differently than the true mean. This translates into a contribution $\left(\tilde{N}_{k}-N_{k}\right)^{2}$ to the error $e(\tilde{p})$. Since the sequence $\left\{\zeta_{t}\right\}_{t \in \mathbb{Z}}$ is i.i.d., the contributions add up to the sum in (18).

The reason why (22) coincides with (20) is as follows. Because of linearity, the derivative in (22) can be computed by setting all shocks $\left\{\zeta_{t^{\prime}}\right\}_{t^{\prime} \leq t-1}$ to zero, except for $\zeta_{t-k}=1$. The shock $\zeta_{t-k}=1$ raises the mean of the state $\theta_{t-k}$ conditional on Period $t-k$ by the regression 
coefficient $G_{1}$. This effect decays over time according to the persistence parameter $\rho$ because all subsequent shocks $\left\{\zeta_{t^{\prime}}\right\}_{t^{\prime}=t-k+1, . ., t-1}$ are zero, i.e., no surprises occur. Therefore, the mean of $\theta_{t-1}$ conditional on Period $t-1$ is raised by $\rho^{k-1} G_{1}$, and the mean of $s_{t}$ is raised by $\rho^{k} G_{1}=C A^{k-1} G=N_{k}$.

The reason why (19) is more complicated than (20) is that after the shock $\zeta_{t-k}=1$, Freddy does not expect the shocks $\left\{\zeta_{t^{\prime}}\right\}_{t^{\prime}=t-k+1, . ., t-1}$ to be zero. This is both because the gambler's fallacy leads him to expect negative shocks, and because he can converge to $\tilde{G}_{1} \neq G_{1}$, thus estimating incorrectly the increase in the state. Because, however, he observes the shocks $\left\{\zeta_{t^{\prime}}\right\}_{t^{\prime}=t-k+1, . ., t-1}$ to be zero, he treats them as surprises and updates accordingly. This generates the extra terms in (19). When $\alpha$ is small, i.e., Freddy is close to rational, the updating generated by $\left\{\zeta_{t^{\prime}}\right\}_{t^{\prime}=t-k+1, . ., t-1}$ is of second order relative to that generated by $\zeta_{t-k}$. The term $\tilde{N}_{k}$ then takes a form analogous to $N_{k}$ :

$$
\tilde{N}_{k} \approx \tilde{C} \tilde{A}^{k} \tilde{G}=\tilde{\rho}^{k} \tilde{G}_{1}-\alpha_{\tilde{\rho}}\left(\delta_{\tilde{\rho}}-\alpha_{\tilde{\rho}}\right)^{k-1} \tilde{G}_{2}
$$

Intuitively, the shock $\zeta_{t-k}=1$ raises Freddy's mean of the state $\theta_{t-k}$ conditional on Period $t-k$ by $\tilde{G}_{1}$, and the effect decays over time at the rate $\tilde{\rho}^{k}$. Freddy also attributes the shock $\zeta_{t-k}=1$ partly to luck through $\tilde{G}_{2}$. He then expects future signals to be lower because of the gambler's fallacy, and the effect decays at the rate $\left(\delta_{\tilde{\rho}}-\alpha_{\tilde{\rho}}\right)^{k} \approx \delta_{\tilde{\rho}}^{k}$.

We conclude this section by determining Tommy's limit posteriors. We examine, in particular, whether Tommy converges to the true parameter values when he initially entertains all values, i.e., $P=P_{0}$. Since Tommy is a Bayesian, his limit posteriors solve the fit-maximization problem for $\alpha=0$.

Proposition 3 Suppose that $\alpha=0$.

- If $\sigma_{\eta}^{2}>0$ and $\rho>0$, then $m\left(P_{0}\right)=\left\{\left(\sigma_{\eta}^{2}, \rho, \sigma_{\omega}^{2}, \mu\right)\right\}$.

- If $\sigma_{\eta}^{2}=0$ or $\rho=0$, then

$$
m\left(P_{0}\right)=\left\{\begin{array}{r}
\left(\tilde{\sigma}_{\eta}^{2}, \tilde{\rho}, \tilde{\sigma}_{\omega}^{2}, \tilde{\mu}\right):\left[\tilde{\sigma}_{\eta}^{2}=0, \tilde{\rho} \in[0,1), \tilde{\sigma}_{\omega}^{2}=\sigma_{\eta}^{2}+\sigma_{\omega}^{2}, \tilde{\mu}=\mu\right] \\
\text { or }\left[\tilde{\sigma}_{\eta}^{2}=0, \tilde{\rho}=1, \tilde{\sigma}_{\omega}^{2}=\sigma_{\eta}^{2}+\sigma_{\omega}^{2}\right] \\
\text { or }\left[\tilde{\sigma}_{\eta}^{2}+\tilde{\sigma}_{\omega}^{2}=\sigma_{\eta}^{2}+\sigma_{\omega}^{2}, \tilde{\rho}=0, \tilde{\mu}=\mu\right]
\end{array}\right\} .
$$

Proposition 3 shows that Tommy converges to the true parameter values if $\sigma_{\eta}^{2}>0$ and $\rho>0$. If $\sigma_{\eta}^{2}=0$ or $\rho=0$, however, then he remains undecided between the true model and 
a set of other models. The intuition is that in both cases signals are i.i.d. in steady state: either because the state converges to a constant value $\left(\sigma_{\eta}^{2}=0\right)$ or because it is not persistent $(\rho=0)$. Therefore, Tommy cannot identify which of $\sigma_{\eta}^{2}$ or $\rho$ is zero. Of course, Tommy's failure to converge to the true model is inconsequential because all models he converges to predict correctly that signals are i.i.d.

\section{Independent Signals}

In this section we consider Freddy's inference problem when signals are i.i.d. As pointed out in the previous section, i.i.d. signals can be generated when $\sigma_{\eta}^{2}=0$ or $\rho=0$. We first study Freddy's "free-form" inference when he initially entertains all parameter values $\left(P=P_{0}\right)$. We next endow Freddy with prior knowledge on the true values of some parameters.

\subsection{No Prior Knowledge}

Proposition 4 characterizes Freddy's convergent beliefs.

Proposition 4 Suppose that $\alpha>0$, and $\sigma_{\eta}^{2}=0$ or $\rho=0$. Then $e\left(P_{0}\right)=0$ and

$$
m\left(P_{0}\right)=\left\{\left(\tilde{\sigma}_{\eta}^{2}, 0, \tilde{\sigma}_{\omega}^{2}, \mu\right): \tilde{\sigma}_{\eta}^{2}+\tilde{\sigma}_{\omega}^{2}=\sigma_{\eta}^{2}+\sigma_{\omega}^{2}\right\}
$$

Since $e\left(P_{0}\right)=0$, Freddy ends up predicting the signals correctly as i.i.d., despite the gambler's fallacy. Intuitively, the fallacy leads him to exaggerate the extent to which a small sample resembles the underlying population - provided that the entire sample is generated by the same distribution. Freddy ends up believing, however, that a different distribution generates the signal in each period. Indeed, the distribution is linked to the state, and Freddy converges to the belief that $\tilde{\rho}=0$, i.e., the state in one period has no relation to the state in the next. In the mutual-fund context, Freddy assumes that managers stay in the fund for only one period. Therefore, his fallacious belief that a given manager's performance should average out over multiple periods has no effect. Formally, when $\tilde{\rho}=0$, the strength $\alpha_{\tilde{\rho}}$ of the gambler's fallacy in (3) is $\alpha_{\tilde{\rho}}=\alpha \tilde{\rho}=0$.

The result of Proposition 4 relies on our interpretation of the gambler's fallacy as applying to the distribution generating the signals for a given state, but not to the distribution 
generating those distributions, i.e., generating the state. For example, Freddy expects that a mutual-fund manager who over-performs in one period is more likely to under-perform in the next. He does not expect, however, that if high-ability managers join the fund in one period, low-ability managers are more likely to follow. The latter belief could lead to incorrect predictions even under a model in which $\tilde{\rho}=0$, i.e., managers stay in the fund for only one period.

We believe that the gambler's fallacy for the state as not as psychologically compelling as for the signals. One reason is that it has a "second-order" flavor, applying to distributions of distributions rather than distributions of signals. Moreover, while the state follows a stochastic process in our model, it could instead be evolving deterministically. Putting these issues aside, the result of Proposition 4 that we wish to emphasize is not Freddy's correct predictions, since predictions are incorrect in the more general settings of Sections 4.2 and 5. A more robust result is that Freddy attempts to explain the absence of systematic reversals by believing that the environment (distribution generating the signals) changes overly frequently. In Proposition 4 this result takes the extreme form that Freddy converges to $\tilde{\rho}=0$, but in Sections 4.2 and 5 convergence is to $\tilde{\rho}$ between zero and the true value $\rho$.

\subsection{Prior Knowledge}

In this section, we allow Freddy to rule out some parameter values based on prior knowledge. Ironically, prior knowledge can hurt Freddy. Indeed, suppose that he knows with confidence that $\tilde{\rho}$ is bounded away from zero. Then, he cannot converge to the belief that $\tilde{\rho}=0$, and consequently cannot predict the signals correctly. Thus, prior knowledge can be harmful because it reduces Freddy's flexibility to come up with the incorrect model that eliminates the gambler's fallacy.

A straightforward example of prior knowledge is when Freddy knows with confidence that the state is constant over time: this translates to the dogmatic belief that $\tilde{\rho}=1$ and $\tilde{\sigma}_{\eta}^{2}=0$. A prototypical occurrence of such a belief is when people observe the flips of a coin they know is fair. The state can be defined as the probability distribution of heads and tails, and is known and constant.

If in our model Freddy has a dogmatic belief that $\tilde{\rho}=1$ and $\tilde{\sigma}_{\eta}^{2}=0$, then he predicts reversals according the gambler's fallacy. This is consistent with the experimental evidence presented in Section 2. Of course, our model matches the evidence by construction, but we 
believe that this is a strength of our approach (in taking the gambler's fallacy as a primitive bias and examining whether the hot-hand fallacy can follow as an implication). Indeed, one could argue that the hot-hand fallacy is a primitive bias, either unconnected to the gambler's fallacy or perhaps even generating it. But then one would have to explain why such a primitive bias does not arise in experiments involving fair coins.

The hot-hand fallacy tends to arise in settings where people are uncertain about the mechanism generating the data, and where a belief that an underlying state varies over time is plausible a priori. Such settings are common when human skill is involved. For example, it is plausible - and often true - that the performance of a basketball player can fluctuate systematically over time because of mood, well-being, etc. Consistent with the evidence, we show below that our approach can generate the hot-hand fallacy in such settings, provided that people are also confident that the state exhibits some persistence.

More specifically, we assume that Freddy allows for the possibility that the state varies over time, but is confident that $\tilde{\rho}$ is bounded away from zero. For example, he can be uncertain as to whether fund managers differ in ability $\left(\tilde{\sigma}_{\eta}^{2}>0\right)$, but know with certainty that they stay in a fund for more than a period (e.g., a month). We take the closed support of Freddy's priors to be

$$
P=P_{\underline{\rho}} \equiv\left\{\left(\tilde{\sigma}_{\eta}^{2}, \tilde{\rho}, \tilde{\sigma}_{\omega}^{2}, \tilde{\mu}\right): \tilde{\sigma}_{\eta}^{2} \in \mathbb{R}^{+}, \tilde{\rho} \in[\underline{\rho}, 1], \tilde{\sigma}_{\omega}^{2} \in \mathbb{R}^{+}, \tilde{\mu} \in \mathbb{R}\right\}
$$

where $\underline{\rho}$ is a lower bound strictly greater than zero and smaller than the true value $\rho$. Note that since $\rho>0$ and signals are assumed i.i.d., the true value $\sigma_{\eta}^{2}$ has to be zero.

To determine Freddy's convergent beliefs, we must minimize the error $e(\tilde{p})$ over the set $P_{\underline{\rho}}$. The problem is more complicated than in Propositions 3 and 4: it cannot be solved by finding parameter vectors $\tilde{p}$ such that $e(\tilde{p})=0$ because no such vectors exist in $P_{\underline{\rho}}$. Instead, we need to evaluate $e(\tilde{p})$ for all $\tilde{p}$ and minimize over $P_{\underline{\rho}}$. Eq. (18) shows that $e(\tilde{p})$ depends on the vector $\tilde{G}$ of regression coefficients, which in turn depends on $\tilde{p}$ in a complicated fashion through the recursive-filtering formulas of Section 3.1. This makes it difficult to solve the problem in closed form. But a closed-form solution can be derived for small $\alpha$, i.e., Freddy close to rational. We next present this solution because it provides useful intuition and has similar properties to the numerical solution for general $\alpha$. 
Proposition 5 Suppose that $\sigma_{\eta}^{2}=0$ and $\rho \geq \rho>0$. When $\alpha$ converges to zero, the set

$$
\left\{\left(\frac{\tilde{\sigma}_{\eta}^{2}}{\alpha \tilde{\sigma}_{\omega}^{2}}, \tilde{\rho}, \tilde{\sigma}_{\omega}^{2}, \tilde{\mu}\right):\left(\tilde{\sigma}_{\eta}^{2}, \tilde{\rho}, \tilde{\sigma}_{\omega}^{2}, \tilde{\mu}\right) \in m\left(P_{\underline{\rho}}\right)\right\}
$$

converges (in the set topology) to the point $\left(z, \underline{\rho}, \sigma_{\omega}^{2}, \mu\right)$, where

$$
z \equiv \frac{\left(1-\underline{\rho}^{2}\right)^{2}}{1-\underline{\rho}^{2} \delta} .
$$

Proposition 5 implies that Freddy's convergent beliefs for small $\alpha$ are $\tilde{p} \approx\left(\alpha z \sigma_{\omega}^{2}, \underline{\rho}, \sigma_{\omega}^{2}, \mu\right)$. Convergence to $\tilde{\rho}=\underline{\rho}$ is intuitive. Indeed, Proposition 4 shows that Freddy attempts to explain the absence of systematic reversals by underestimating the state's persistence $\tilde{\rho}$. The smallest value of $\tilde{\rho}$ consistent with the prior knowledge that $\tilde{\rho} \in[\underline{\rho}, 1]$ is $\underline{\rho}$.

Freddy's belief that $\tilde{\rho}=\underline{\rho}$ leaves him unable to explain fully the absence of reversals. To generate a fuller explanation, he develops the additional fallacious belief that $\tilde{\sigma}_{\eta}^{2} \approx \alpha z \sigma_{\omega}^{2}>0$, i.e., the state varies over time. Thus, in a mutual-fund context, he overestimates both the extent of managerial turnover and that of differences in ability. Overestimating turnover helps him explain the absence of reversals in fund returns because he believes that reversals concern only the performance of individual managers. Overestimating differences in ability helps him further because he can attribute streaks of high or low fund returns to individual managers being above or below average. As we show below, the belief in the changing state can generate a hot-hand fallacy: Freddy can predict that streaks of high or low returns will continue despite all managers being average.

The error-minimization problem has a useful graphical representation. Consider Freddy's expectation of $s_{t}$ conditional on Period $t-1$, as a function of the past signals. Eq. (23) shows that the effect of the signal in Period $t-k$, holding other signals to their mean, is $\tilde{N}_{k}$. Eq. (24) expresses $\tilde{N}_{k}$ as the sum of two terms. Subsequent to a high $s_{t-k}$, Freddy believes that the state has increased, which raises his expectation of $s_{t}\left(\operatorname{term} \tilde{\rho}^{k} \tilde{G}_{1}\right)$. But he also believes that luck should reverse, which lowers his expectation $\left(\operatorname{term}-\alpha_{\tilde{\rho}}\left(\delta_{\tilde{\rho}}-\alpha_{\tilde{\rho}}\right)^{k-1} \tilde{G}_{2}\right)$. Figure 1 plots these terms (dotted and dashed line, respectively) and their sum $\tilde{N}_{k}$ (solid line), as a function of the lag $k$. Since signals are i.i.d. under the true model, $N_{k}=0$. Therefore, minimizing the infinite sum in (18) amounts to finding $\left(\frac{\tilde{\sigma}_{n}^{2}}{\tilde{\sigma}_{\omega}^{2}}, \tilde{\rho}\right)$ that minimize the average squared distance between the solid line and the $x$-axis. 


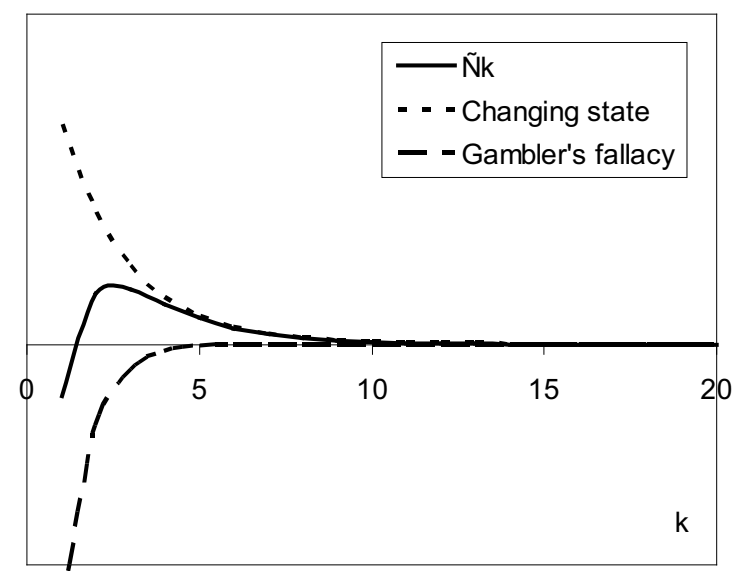

Figure 1: Effect of a signal in Period $t-k$ on Freddy's expectation $\tilde{E}_{t-1}\left(s_{t}\right)$, as a function of $k$. The dotted line represents the belief that the state has changed, the dashed line represents the effect of the gambler's fallacy, and the solid line is $\tilde{N}_{k}$, the sum of the two effects.

Figure 1 shows that $\tilde{N}_{k}$ is not always of the same sign. Thus, a high past signal does not lead Freddy to predict always a high or always a low signal. Suppose instead that he always predicts a low signal because the gambler's fallacy dominates the belief that the state has increased (i.e., the dotted line is uniformly closer to the $x$-axis than the dashed line). This means that he converges to a small value of $\tilde{\sigma}_{\eta}^{2}$, believing that the state's variation is small, and treating signals as not very informative. But then, a larger value of $\tilde{\sigma}_{\eta}^{2}$ would shift the dotted line up, reducing the average distance between the solid line and the $x$-axis.

The change in $\tilde{N}_{k}$ 's sign is from negative to positive. Thus, a high signal in the recent past leads Freddy to predict a low signal, while a high signal in the more distant past leads him to predict a high signal. This is because the belief that the state has increased decays at the rate $\tilde{\rho}^{k}$, while the effect of the gambler's fallacy decays at the faster rate $\left(\delta_{\tilde{\rho}}-\alpha_{\tilde{\rho}}\right)^{k}=\tilde{\rho}^{k}(\delta-\alpha)^{k}$. In other words, after a high signal Freddy expects luck to reverse quickly but views the increase in the state as more long-lived. The reason why he expects luck to reverse quickly relative to the state is that he views luck as specific to a given state (e.g., a given fund manager).

We next draw the implications of our results for Freddy's predictions after streaks. We consider a streak of identical signals between Periods $t-k$ and $t-1$.

Proposition 6 Suppose that $\alpha$ is small, $\sigma_{\eta}^{2}=0, \rho \geq \underline{\rho}>0$, and Freddy considers parameter 
values in the set $P_{\underline{\rho}}$. Then, in steady state

$$
\sum_{k^{\prime}=1}^{k} \frac{\partial \tilde{E}_{t-1}\left(s_{t}\right)}{\partial s_{t-k^{\prime}}}
$$

is negative for $k=1$ and becomes positive as $k$ increases.

Proposition 6 shows that Freddy predicts a low signal following a short streak of high signals, but a high signal following a longer streak. Thus, the gambler's fallacy dominates after short streaks but the hot-hand fallacy appears after longer streaks. This is consistent with Figure 1 because the effect of a streak is the sum of the effects $\tilde{N}_{k}$ of each signal in the streak. Since $\tilde{N}_{k}$ is negative for small $k$, Freddy predicts a low signal following a short streak. But as streak length increases, the positive values of $\tilde{N}_{k}$ overtake the negative values, generating a positive cumulative effect.

Propositions 5 and 6 make use of the closed-form solutions derived for small $\alpha$. For general $\alpha$, the fit-maximization problem can be solved numerically and the results confirm the closed-form solutions: Freddy converges to $\tilde{\sigma}_{\eta}^{2}>0, \tilde{\rho}=\underline{\rho}$, and $\tilde{\mu}=\mu$, and his predictions after streaks are as in Proposition 6. ${ }^{14}$

\section{$5 \quad$ Serially Correlated Signals}

In this section we consider Freddy's inference problem when the signals are serially correlated. Serial correlation arises when the state varies over time $\left(\sigma_{\eta}^{2}>0\right)$ and is persistent $(\rho>0)$. To highlight the new effects relative to the i.i.d. case, we assume that Freddy has no prior knowledge on parameter values.

Recall that with i.i.d. signals and no prior knowledge, Freddy predicts correctly because he converges to the belief that $\tilde{\rho}=0$, i.e., the state in one period has no relation to the state in the next. When signals are serially correlated, the belief $\tilde{\rho}=0$ obviously generates incorrect predictions. But predictions are also incorrect under a belief $\tilde{\rho}>0$ because the gambler's fallacy then takes effect. Therefore, there is no parameter vector $\tilde{p} \in P_{0}$ achieving zero error $e(\tilde{p}) \cdot{ }^{15}$

\footnotetext{
${ }^{14}$ The result that $\tilde{\sigma}_{\eta}^{2}>0$ can be shown analytically. The proof is available upon request.

${ }^{15}$ The proof of this result is available upon request.
} 
To minimize the error, we proceed as in Section 4.2, evaluating $e(\tilde{p})$ for all $\tilde{p}$ and minimizing over $P_{0}$. We solve this problem in closed form for small $\alpha$ and compare with the numerical solution for general $\alpha$. In addition to $\alpha$, we take the variance $\sigma_{\eta}^{2}$ of the shocks to the state to be small, meaning that signals are close to i.i.d. We set $\nu \equiv \sigma_{\eta}^{2} /\left(\alpha \sigma_{\omega}^{2}\right)$ and assume that $\alpha$ and $\sigma_{\eta}^{2}$ converge to zero holding $\nu$ constant. The case where $\sigma_{\eta}^{2}$ remains constant while $\alpha$ converges to zero can be derived as a limit for $\nu=\infty$.

Proposition 7 Suppose that $\rho>0$. When $\alpha$ and $\sigma_{\eta}^{2}$ converge to zero, holding $\nu$ constant, the set

$$
\left\{\left(\frac{\tilde{\sigma}_{\eta}^{2}}{\alpha \tilde{\sigma}_{\omega}^{2}}, \tilde{\rho}, \tilde{\sigma}_{\omega}^{2}, \tilde{\mu}\right):\left(\tilde{\sigma}_{\eta}^{2}, \tilde{\rho}, \tilde{\sigma}_{\omega}^{2}, \tilde{\mu}\right) \in m\left(P_{0}\right)\right\}
$$

converges (in the set topology) to the point $\left(z, r, \sigma_{\epsilon}^{2}, \mu\right)$, where

$$
z \equiv \frac{\nu \rho\left(1-r^{2}\right)^{2}}{r\left(1-\rho^{2}\right)(1-\rho r)}+\frac{\left(1-r^{2}\right)^{2}}{1-r^{2} \delta}
$$

and $r$ solves

$$
\frac{\nu \rho(\rho-r)}{\left(1-\rho^{2}\right)(1-\rho r)^{2}} H_{1}(r)=\frac{r^{2}(1-\delta)}{\left(1-r^{2} \delta\right)^{2}} H_{2}(r)
$$

for

$$
\begin{aligned}
H_{1}(r) & \equiv \frac{\nu \rho}{\left(1-\rho^{2}\right)(1-\rho r)}+\frac{r(1-\delta)\left[2-\rho r(1+\delta)-r^{2} \delta+\rho^{2} r^{4} \delta^{2}\right]}{\left(1-r^{2} \delta\right)^{2}(1-\rho r \delta)^{2}} \\
H_{2}(r) & \equiv \frac{\nu \rho}{\left(1-\rho^{2}\right)(1-\rho r)}+\frac{r(1-\delta)\left(2-r^{2} \delta^{2}-r^{4} \delta^{3}\right)}{\left(1-r^{2} \delta\right)\left(1-r^{2} \delta^{2}\right)^{2}}
\end{aligned}
$$

Because $H_{1}(r)$ and $H_{2}(r)$ are positive, (27) implies that $r \in(0, \rho)$. Thus, Freddy converges to a persistence parameter $\tilde{\rho}=r$ that is between zero and the true value $\rho$. As in Section 4, Freddy underestimates $\tilde{\rho}$ in his attempt to explain the absence of systematic reversals. But he does not converge all the way to $\tilde{\rho}=0$ because he must explain the signals' serial correlation. Consistent with intuition, $\tilde{\rho}$ is close to zero when the gambler's fallacy is strong relative to the serial correlation ( $\nu$ small), and is close to $\rho$ in the opposite case.

Consider next Freddy's estimate $\tilde{\sigma}_{\eta}^{2}$ of the variance of the shocks to the state. Section 4.2 shows that when the true variance $\sigma_{\eta}^{2}$ is zero, Freddy can develop the fallacious belief that $\tilde{\sigma}_{\eta}^{2}>0$ as a way to counteract the effect of the gambler's fallacy. When $\sigma_{\eta}^{2}$ is positive, we find the analogous result that Freddy overestimates $\tilde{\sigma}_{\eta}^{2}$. Indeed, he converges to $\tilde{\sigma}_{\eta}^{2} \approx \alpha z \sigma_{\omega}^{2}=$ $(z / \nu) \sigma_{\eta}^{2}$, which is larger than $\sigma_{\eta}^{2}$ because of (26) and $r<\rho$. Note that $z$ in (26) is decreasing 
in $r$. Thus, Freddy overestimates $\tilde{\sigma}_{\eta}^{2}$ partly as a way to compensate for underestimating $\tilde{\rho}$.

The error minimization problem can be represented graphically. Consider Freddy's expectation of $s_{t}$ conditional on Period $t-1$, as a function of the past signals. The effect of the signal in Period $t-k$, holding other signals to their mean, is $\tilde{N}_{k}$. Figure 2 plots $\tilde{N}_{k}$ (solid line) as a function of $k$. It also decomposes $\tilde{N}_{k}$ to the belief that the state has increased (dotted line) and the effect of the gambler's fallacy (dashed line). The new element relative to Figure 1 is that an increase in $s_{t-k}$ also affects the expectation $E_{t-1}\left(s_{t}\right)$ under the true model. This effect, $N_{k}$, is represented by the solid line with diamonds. Minimizing the infinite sum in (18) amounts to finding $\left(\frac{\tilde{\sigma}_{n}^{2}}{\tilde{\sigma}_{\omega}^{2}}, \tilde{\rho}\right)$ that minimize the average squared distance between the solid line and the solid line with diamonds.

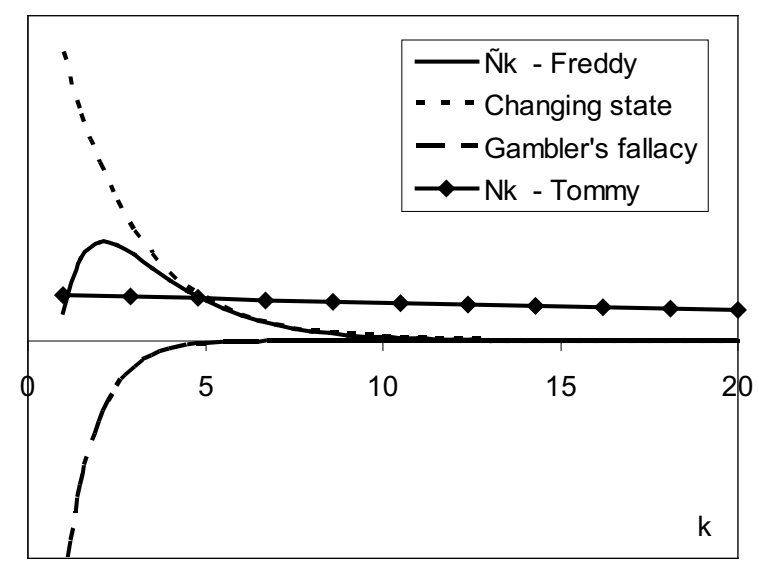

Figure 2: Effect of a signal in Period $t-k$ on Freddy's expectation $\tilde{E}_{t-1}\left(s_{t}\right)$, as a function of $k$. The dotted line represents the belief that the state has changed, the dashed line represents the effect of the gambler's fallacy, and the solid line is $\tilde{N}_{k}$, the sum of the two effects. The solid line with diamonds is $N_{k}$, the effect on the expectation $E_{t-1}\left(s_{t}\right)$ under the true model.

For large $k, \tilde{N}_{k}$ is below $N_{k}$, meaning that Freddy under-reacts to signals in the distant past. This is because he underestimates the state's persistence parameter $\tilde{\rho}$, thus believing that the information learned from signals about the state becomes obsolete overly fast. Note that under-reaction to distant signals is a novel feature of the serial-correlation case. Indeed, with i.i.d. signals, Freddy's underestimation of $\tilde{\rho}$ does not lead to under-reaction because there is no reaction under the true model.

Freddy's reaction to signals in the more recent past is in line with the i.i.d. case. Since $\tilde{N}_{k}$ cannot be below $N_{k}$ uniformly (otherwise $e(\tilde{p})$ could be made smaller for a larger value of $\tilde{\sigma}_{\eta}^{2}$ ), it has to exceed $N_{k}$ for smaller values of $k$. Thus, Freddy over-reacts to signals in the 
more recent past. The intuition is as in Section 4.2: in overestimating $\tilde{\sigma}_{\eta}^{2}$, Freddy exaggerates the signals' informativeness about the state. Finally, Freddy under-reacts to signals in the very recent past because of the gambler's fallacy. Proposition 8 examines the implications of these results for predictions after streaks.

Proposition 8 Suppose that $\alpha$ and $\sigma_{\eta}^{2}$ are small, $\rho>0$, and Freddy has no prior knowledge $\left(P=P_{0}\right)$. Then, in steady state

$$
\sum_{k^{\prime}=1}^{k} \frac{\partial \tilde{E}_{t-1}\left(s_{t}\right)}{\partial s_{t-k^{\prime}}}-\sum_{k^{\prime}=1}^{k} \frac{\partial E_{t-1}\left(s_{t}\right)}{\partial s_{t-k^{\prime}}}
$$

is negative for $k=1$, becomes positive as $k$ increases, and then becomes negative again.

Proposition 8 shows that Freddy under-reacts to short streaks, over-reacts to longer streaks, and under-reacts again to very long streaks. The under-reaction to short streaks is because of the gambler's fallacy. Longer streaks generate over-reaction because Freddy overestimates the signals' informativeness about the state. But he also underestimates the state's persistence, thus under-reacting to very long streaks.

The numerical results for general $\alpha$ confirm most of the closed-form results. The only exception is that $\tilde{N}_{k}-N_{k}$ can change sign only once, from positive to negative. Under-reaction then occurs only to very long streaks. This tends to happen when Freddy underestimates the state's persistence significantly (because $\alpha$ is large relative to $\tilde{\sigma}_{\eta}^{2}$ ). As a way to compensate for his error, he overestimates $\tilde{\sigma}_{\eta}^{2}$ significantly, viewing signals as very informative about the state. Even very short streaks can then lead him to believe that the change in the state is large and dominates the effect of the gambler's fallacy.

\section{Finance Applications}

Sections 4 and 5 show that the gambler's fallacy can influence Freddy's perception of several aspects of his environment. In particular, he can underestimate the state's persistence and overestimate the signals' informativeness about the state. While these errors help to counteract the fallacious belief in reversals, they generally do not offset it fully, leading to incorrect predictions. We next explore the implications of these results for economic decisions. Our 
goal is to show that the gambler's fallacy can have a wide range of implications, and that our normal-linear model is a useful tool for pursuing them.

One way to map Freddy's predictions into economic decisions is to interpret signals as financial returns and Freddy as an investor. Suppose that in Period $t-1$ Freddy can invest in a risky asset with return $s_{t}$ and a riskless asset with return $r$. For simplicity, we assume that Freddy has an one-period horizon, maximizing expected utility over Period $t$ wealth. ${ }^{16}$ To keep with the normal-linear structure, we take utility over wealth to be exponential with coefficient of absolute risk aversion $a$. Finally, we assume that we are in steady state. Denoting wealth in Period $t^{\prime} \in\{t-1, t\}$ by $\tilde{W}_{t^{\prime}}$, and the investment in the risky asset by $\tilde{X}_{t-1}$, the maximization problem is

$$
\max _{\tilde{X}_{t-1}} \tilde{E}_{t-1}\left[-\exp \left(-a \tilde{W}_{t}\right)\right]
$$

subject to the budget constraint

$$
\tilde{W}_{t}=\left(\tilde{W}_{t-1}-\tilde{X}_{t-1}\right)(1+r)+\tilde{X}_{t-1}\left(1+s_{t}\right)
$$

Because of normality and exponential utility, the problem is mean-variance and the optimal investment is

$$
\tilde{X}_{t-1}=\frac{\tilde{E}_{t-1}\left(s_{t}\right)-r}{a \tilde{V} a r_{t-1}\left(s_{t}\right)},
$$

where $\tilde{V}$ ar denotes the variance assessed by Freddy. Note that in steady state the variance $\tilde{V} a r_{t-1}\left(s_{t}\right)$ is constant (equal to $\sigma_{s}^{2}(\tilde{p})$ ). We denote this constant by $\tilde{V} a r_{1}$, setting more generally $\tilde{V} a r_{k} \equiv \tilde{V} a r_{t-k}\left(s_{t}\right)$. Using (29), we can compute several quantities of interest.

\subsection{Trading Volume}

The change in Freddy's investment between Periods $t-1$ and $t$ is

$$
\Delta \tilde{X}_{t} \equiv \tilde{X}_{t}-\tilde{X}_{t-1}=\frac{\tilde{E}_{t}\left(s_{t+1}\right)-\tilde{E}_{t-1}\left(s_{t}\right)}{a \tilde{V} a r_{1}} .
$$

\footnotetext{
${ }^{16} \mathrm{~A}$ literal interpretation of this assumption is that there are overlapping generations of investors, each of whom lives for one period but observes the entire history of past signals. Alternatively, we can view the assumption as a simplification of the infinite-horizon problem that considers only the myopic demand and ignores the demand for intertemporal hedging (Merton (1971)).
} 
Thus, Freddy changes his investment if he believes that expected return has changed. The quantity $\Delta \tilde{X}_{t}$ is a measure of Freddy's trading activity in Period $t$, and we define trading volume $\tilde{Q}_{t}$ as the absolute value $\left|\Delta \tilde{X}_{t}\right| \cdot{ }^{17}$ We also define average volume $\tilde{Q}$ as the unconditional expectation of $\tilde{Q}_{t}$, i.e., $\tilde{Q} \equiv E\left(\tilde{Q}_{t}\right)$. Proposition 9 computes Freddy's average volume and compares it to that of a Bayesian investor (Tommy) with the same preferences. The comparison is made for two settings in which Freddy converges to incorrect predictions: i.i.d. returns and prior knowledge that $\tilde{\rho}$ is bounded away from zero (Section 4.2), and serially correlated returns and no prior knowledge (Section 5).

Proposition 9 Freddy's average volume is

$$
\tilde{Q}=\sqrt{\frac{2}{\pi}} \frac{\sigma_{s}}{a \tilde{V} a r_{1}} \sqrt{\sum_{k=1}^{\infty}\left(\tilde{N}_{k}-\tilde{N}_{k-1}\right)^{2}}
$$

where $\tilde{N}_{0} \equiv 0$. Tommy's average volume $Q$ is given by (31) without the tildes and where $N_{0} \equiv 0$.

- Suppose that $\sigma_{\eta}^{2}=0$ and Freddy has prior knowledge that $\tilde{\rho} \geq \underline{\rho}$ for $\underline{\rho} \in(0, \rho]$. Then $\tilde{Q}>Q=0$.

- Suppose that $\alpha$ and $\sigma_{\eta}^{2}$ are small, $\rho>0$, and Freddy has no prior knowledge $\left(P=P_{0}\right)$. Then $\tilde{Q}>Q$ if $\nu \equiv \sigma_{\eta}^{2} / \alpha \sigma_{\omega}^{2}$ is small, and $\tilde{Q}<Q$ if $\nu$ is large.

The result in the i.i.d. case is straightforward. Since expected returns are constant, Tommy's volume is zero. But if Freddy has prior knowledge that $\tilde{\rho}$ is bounded away from zero, then he ends up believing that expected returns are time-varying. Thus, his volume is positive and exceeds Tommy's. Note that this holds independently of Freddy's prior knowledge on $\tilde{\sigma}_{\eta}^{2}$, i.e., whether or not he ends up believing in the hot hand.

The result in the serial-correlation case is more subtle than in the i.i.d. case both because Tommy's volume is non-zero and because Freddy is assumed to have no prior knowledge. To explain the intuition, we return to Figure 2 of Section 5, and plot it for two different

\footnotetext{
${ }^{17}$ Our definition of trading volume assumes that the return $s_{t}$ is due only to dividends. Suppose that $s_{t}=s_{t}^{d}+s_{t}^{c}$, where $s_{t}^{d}$ is due to dividends and $s_{t}^{c}$ to capital gains. Then, if Freddy buys shares worth $\tilde{X}_{t-1}$ in Period $t-1$, he receives a dividend $\tilde{X}_{t-1} s_{t}^{d}$ in Period $t$, and his shares are worth $\tilde{X}_{t-1} s_{t}^{c}$. Thus, his net trade in Period $t$ is $\tilde{X}_{t}-\tilde{X}_{t-1}\left(1+s_{t}^{c}\right)$ and coincides with $\tilde{X}_{t}-\tilde{X}_{t-1}$ only when $s_{t}^{c}=0$. When $s_{t}^{c} \neq 0, \tilde{X}_{t}-\tilde{X}_{t-1}$ represents the component of the net trade that is due to changes in expectations about future returns.
} 
values of $\nu$ as Figure 3 below. In each plot, the solid line represents the effect $\tilde{N}_{k}$ of the return $k-1$ periods ago on Freddy's forecast of next period's return, and the solid line with diamonds represents the effect $N_{k}$ for Tommy. Note that $\tilde{N}_{k}$ is also the effect on Freddy's investment, because the investment is linear in the forecast. Moreover, $\tilde{N}_{k}-\tilde{N}_{k-1}$ is the effect on Freddy's net trade, provided that we set $\tilde{N}_{0} \equiv 0$ to cover the case $k=1$.
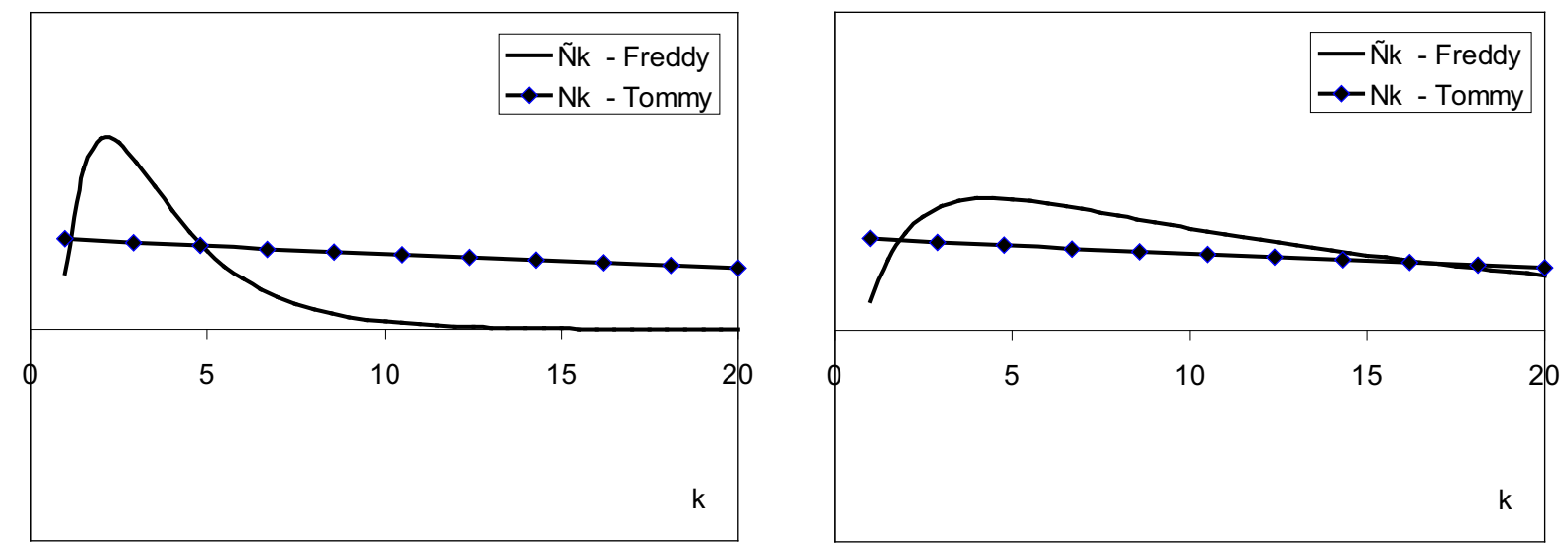

Figure 3: The solid line represents the effect $\tilde{N}_{k}$ of the return $k-1$ periods ago on Freddy's forecast of next period's return. The solid line with diamonds represents the effect $N_{k}$ for Tommy. The plot to the left is for small $\nu \equiv$ $\sigma_{\eta}^{2} / \alpha \sigma_{\omega}^{2}$, and the plot to the right is for large $\nu$.

Consider now Freddy's net trade in Period $t$ as a function of the history of past returns. This function is linear, and the coefficient of the return innovation $\zeta_{t-k+1}$ is $\tilde{N}_{k}-\tilde{N}_{k-1}$. Since innovations are i.i.d., the variance of net trade can be derived by squaring the coefficients and adding them up. The volume is equal to the square root of the variance (times a constant) because of normality. Thus, comparing Freddy's volume to Tommy's amounts to comparing the average squared slope of the lines in Figure 3, where the average is taken over $k$, the slope for $k$ is defined as the value for $k$ minus the value for $k-1$, and the value for $k=0$ is taken to be zero.

The plot to the left in Figure 3 is for small $\nu$, i.e., strong gambler's fallacy relative to serial correlation. In that case, Freddy converges to a small value of the persistence parameter $\tilde{\rho}$ and a large value of $\tilde{\sigma}_{\eta}^{2} / \sigma_{\eta}^{2}$, i.e., treats signals as very informative about the state but also believes that the information becomes fast obsolete (Proposition 7). Following a high signal, Freddy establishes a smaller initial position that Tommy because he expects a short-term 
reversal of luck. Once the expectation of the reversal subsides, he builds the position to a level larger than Tommy because he treats the signal as very positive news. But he also believes that news are short-lived, and unwinds the position rapidly. Because of the large position and the rapid unwinding, Freddy's trades are large, and the sum of squared slopes is larger than for Tommy. On the other hand, when $\nu$ is large, as in the plot to the right, Freddy builds and unwinds the position slowly. The sum of squared slopes is then smaller than for Tommy because of Tommy's larger initial trade.

Turning to applications, suppose that $s_{t}$ is the return on a mutual fund, in which case our volume variable can be interpreted as the flow in or out of the fund. A puzzling fact about fund flows is that they are strongly related to funds' lagged returns (e.g., Chevalier and Ellison (1997), Sirri and Tufano (1998)) even though the latter do not appear to be strong predictors of future returns (e.g., Carhart (1997)). ${ }^{18}$ Berk and Green (2004) propose a rational explanation for this puzzle. They assume that ability differs across managers and can be inferred from returns. Able managers perform well and receive inflows, but because of decreasing returns to managing a large fund, their performance drops to that of average managers. This explanation, however, requires large ability differentials to match the empirical flow-performance relationship: the standard deviation of the ability distribution must correspond to an annual return of $6 \%$, meaning that in the absence of decreasing returns, top-quartile managers would outperform bottom-quartile ones by more than $15 \%$.

Our results suggest that return predictability or differences in managerial ability do not have to be overly large to match the extent of performance-driven fund flows. Indeed, Proposition 9 shows that Freddy generates non-zero flows even in the absence of ability differences, as long as he is confident that ability in consecutive periods is related ( $\tilde{\rho}$ bounded away from zero). ${ }^{19}$ Even when Freddy does not hold such a confident belief, his flows exceed Tommy's if differences in ability exist and are not too large. We return to this issue in the calibrated example of Section 6.4, where we show that Freddy's flows exceed Tommy's for

\footnotetext{
${ }^{18}$ See also Baquero and Verbeek (2006) who regress hedge-fund flows on lagged returns and a proxy for the lagged returns' true predictive power about future returns. They find that lagged returns have an effect above and beyond their ability to predict future returns.

${ }^{19}$ The i.i.d. case generates the counterfactual implication that good performance leads to short-run outflows, because Freddy expects luck to reverse. One way to address this issue is to focus on the case of serial correlation (and no prior knowledge), where the flow-performance relationship is generally positive even in the short run. But the issue could also be addressed within the i.i.d. framework if, for some reason, Freddy's estimate of $\tilde{\sigma}_{\eta}^{2}$, i.e., of differences in ability, is larger than the one derived in Section 4.1. In Section 6.2 we propose one reason: a fallacious belief in financial expertise that Freddy can develop if he overestimates the value of fund managers' information.
} 
plausible parameter values.

\subsection{Value of Information}

Our analysis so far assumes that signals are freely available. We next examine how much Freddy would be willing to pay for them, and how this compares to Tommy's valuation. We denote by $\tilde{U}_{1}\left(\tilde{W}_{t-1}, \tilde{E}_{t-1}\left(s_{t}\right)\right)$ the maximum in $(28)$, i.e., Freddy's maximum utility when he observes all returns up to Period $t-1$. We also denote by $\tilde{U}_{k}\left(\tilde{W}_{t-1}, \tilde{E}_{t-k}\left(s_{t}\right)\right)$ Freddy's maximum utility when he chooses investment in Period $t-1$ but observes returns up to Period $t-k$ only (in which case his expectation of $s_{t}$ is $\tilde{E}_{t-k}\left(s_{t}\right)$ ). If Freddy pays $\tilde{I}_{k}$ to observe the $k$ most recent returns $\left\{s_{t^{\prime}}\right\}_{t^{\prime}=t-k, . ., t-1}$, then he will receive the utility $\tilde{U}_{1}\left(\tilde{W}_{t-1}-\tilde{I}_{t-1}, \tilde{E}_{t-1}\left(s_{t}\right)\right)$. The value $\tilde{I}_{k}$ of the information in the $k$ most recent returns is defined by

$$
\tilde{U}_{k+1}\left(\tilde{W}_{t-1}, \tilde{E}_{t-k-1}\left(s_{t}\right)\right)=\tilde{E}_{t-k-1} \tilde{U}_{1}\left(\tilde{W}_{t-1}-\tilde{I}_{k}, \tilde{E}_{t-1}\left(s_{t}\right)\right) .
$$

Because of normality and exponential utility, $\tilde{I}_{k}$ takes a very simple form. In particular, it does not depend on the realization of $\tilde{E}_{t-k-1}\left(s_{t}\right)$, nor on Freddy's wealth.

Proposition 10 Freddy's value of observing the $k$ most recent returns is

$$
\tilde{I}_{k}=\frac{1}{2 a(1+r)} \log \left(\frac{\tilde{V} a r_{k+1}}{\tilde{V} a r_{1}}\right)=\frac{1}{2 a(1+r)} \log \left(1+\sum_{k^{\prime}=1}^{k}\left(\tilde{C} \tilde{A}^{k^{\prime}-1} \tilde{G}\right)^{2}\right)
$$

Tommy's value $I_{k}$ is given by (33) without the tildes.

- Suppose that $\sigma_{\eta}^{2}=0$ and Freddy has prior knowledge that $\tilde{\rho} \geq \underline{\rho}$ for $\underline{\rho} \in(0, \rho]$. Then $I_{k}=0$ for all $k \geq 1$, and $\tilde{I}_{k}>0$ for all $k \geq 2$.

- Suppose that $\alpha$ and $\sigma_{\eta}^{2}$ are small, $\rho>0$, and Freddy has no prior knowledge $\left(P=P_{0}\right)$. Then there exists $k$ such that $\tilde{I}_{k}>I_{k}$. Moreover, $\tilde{I}_{\infty}<I_{\infty}$ if $\nu$ is small, and $\tilde{I}_{\infty}>I_{\infty}$ if $\nu$ is large.

The result in the i.i.d. case is straightforward. Since past returns do not predict future ones, Tommy's value of information is zero. But if Freddy has prior knowledge that $\tilde{\rho}$ is bounded away from zero, then he ends up believing that returns are non-i.i.d. and treats past returns as valuable information. 
To explain the intuition in the serial-correlation case, we return to Figure 3 that represents the effect of past returns on Freddy's and Tommy's forecast of next period's return. Intuitively, the larger the effect is, the larger the value of observing past returns should be. Eq. (33) confirms this intuition. For example, Tommy's value of information is an increasing function of $\sum_{k^{\prime}=1}^{k}\left(C A^{k^{\prime}-1} G\right)^{2}$. This term is equal (from $(20)$ ) to $\sum_{k^{\prime}=1}^{k} N_{k^{\prime}}^{2}$, i.e., the sum of squared effects of the $k$ most recent returns on Tommy's forecast. Freddy's value of information is the same increasing function of $\sum_{k^{\prime}=1}^{k}\left(\tilde{C} \tilde{A}^{k^{\prime}-1} \tilde{G}\right)^{2}$, and for small $\alpha$ this term is approximately equal (from $(24)$ ) to $\sum_{k^{\prime}=1}^{k} \tilde{N}_{k^{\prime}}^{2}{ }^{20}$ Thus, for small $\alpha$, comparing the value of information for Freddy and Tommy amounts to comparing the average squared height of the first $k$ points of the lines in Figure 3.

Proposition 10 shows that for some values of $k$, the average height of the first $k$ points on Freddy's line is larger than for Tommy's line. Thus, Freddy overestimates the informational value of recent return histories, and this is because he overestimates the information that returns convey about the state. ${ }^{21}$ But because he believes information to become fast obsolete, he underestimates the value of returns in the distant past. This can lead him to undervalue the full return history. Such undervaluation occurs for small $\nu$, i.e., strong gambler's fallacy relative to serial correlation, which is when Freddy underestimates significantly the state's persistence. Figure 3 provides a graphical interpretation: because Freddy's line in the plot to the left converges to zero quickly, its average height is smaller than for Tommy's line.

Turning to applications, suppose that $s_{t}$ is the i.i.d. return on a traded asset (e.g., stock, bond). Suppose also that Freddy is confident that expected returns in consecutive periods are related. Then, because he ends up treating returns as non-i.i.d., he can believe in technical analysis (chartism), whereby past returns are used to predict the future. Moreover, if he does not observe the detailed return history, he can view agents who do so (e.g., mutual-fund managers, stockbrokers) as valuable experts. This could be one explanation why individuals invest in actively-managed funds, even though the funds do not seem to outperform their market benchmarks. ${ }^{22}$ Note that if Freddy develops a belief in financial expertise, he can also believe that ability differences between managers are potentially large. This could reinforce

\footnotetext{
${ }^{20}$ The intuition why the equality for Freddy is approximate is that his value of information involves the effects under his model $\left(\tilde{C} \tilde{A}^{k-1} \tilde{G}\right)$ and not under the true model $\left(\tilde{N}_{k}\right)$. For small $\alpha$, the effects coincide approximately.

${ }^{21}$ Freddy can underestimate the informational value of the most recent return because of his initial underreaction. For some parameter values, however, the under-reaction can be so severe as to make the effect of the most recent return negative and larger in absolute value than for Tommy.

${ }^{22}$ See Fama (1991) for a survey of the evidence.
} 
the implications of Section 6.1 on the flow-performance relationship. ${ }^{23}$

\subsection{Equilibrium Prices}

We next examine how Freddy's fallacious beliefs can affect equilibrium prices. Recall from Lemma 2 that when Freddy predicts the signals erroneously, he overestimates their variance because he attributes his prediction error to signal noise. His average investment is then lower than Tommy's, meaning that the risk premium in a market with Freddies can be higher than with Tommies. ${ }^{24}$ This could perhaps speak to the equity-premium puzzle (Mehra and Prescott (1985)) that stocks' expected return is high relative to risk.

A second implication provides a link between our work and Barberis, Shleifer and Vishny (BSV 1998). In BSV, investors do not realize that innovations to a company's earnings are i.i.d., but believe them to be drawn either from a regime with excess reversals or from one with excess streaks. If the reversal regime is the more common, the stock price underreacts to short streaks because investors expect a reversal. The price over-reacts, however, to longer streaks because investors take them as sign of a switch to the streak regime. This can generate short-run momentum and long-run reversals in stock returns, consistent with the empirical evidence (surveyed in BSV). Section 4.2 has similar implications because it shows that in i.i.d. environments Freddy can under-react to short streaks and over-react to longer ones. But while the implications are similar, our approach is different. In particular, BSV provide a psychological foundation for their assumptions by appealing to a combination of biases: the conservatism bias for the reversal regime and the representativeness bias for the streak regime. Our model, by contrast, not only derives such biases from the single underlying bias of the gambler's fallacy, but in doing so provides predictions as to which biases are the most relevant in different informational settings.

\footnotetext{
${ }^{23}$ The implications on financial expertise rely on Freddy's confident belief that $\tilde{\rho}$ is bounded away from zero. Without such a belief, Freddy predicts correctly in an i.i.d. environment, and can underestimate the informational value of the return history under serial correlation. A result that does not rely on beliefs about $\tilde{\rho}$ is the overestimation of the value of recent returns. Thus, investors could be overpaying for newsletters that analyze the recent performance of stocks or mutual funds, while underpaying for long-horizon studies.

${ }^{24}$ Formally, (29) implies that Freddy's average investment in the risky asset is $E\left(\tilde{X}_{t}\right)=(\mu-r) /\left(a \tilde{V} a r_{1}\right)$. Since Lemma 2 implies that $\tilde{V} a r_{1}=\operatorname{Var}_{1}+e(\tilde{p})$, Freddy's average investment is lower than Tommy's as long as $e(\tilde{p})>0$ and $\mu>r$. Note a surprising implication of our results in the case of $i . i . d$. returns and prior knowledge about $\tilde{\rho}$ : Freddy views returns as predictable and yet after conditioning on the predictive information views returns as more uncertain than they truly are.
} 


\subsection{Calibration}

We conclude with a calibration exercise that serves both to compute the effects that our model generates for plausible parameter values and confirm the robustness of the closedform results derived for small $\alpha$. We take periods to be months, meaning that investors check the performance of their fund managers once a month. We set $\rho=0.98$, meaning that shocks to managerial ability have a half-life of approximately three years $\left(\rho^{36}=0.48\right)$. Such a half-life seems reasonable: for example, Jagannathan, Malakhov and Novikov (JMN 2006) estimate that approximately half of the abnormal performance of a hedge fund over a three-year period spills over to the next three years. We calibrate $\sigma_{\eta}^{2} / \sigma_{\omega}^{2}$ through the ratio

of unconditional variances of $\theta_{t}$ and $s_{t}$, which is $\frac{\sigma_{\eta}^{2} /\left(1-\rho^{2}\right)}{\sigma_{\eta}^{2} /\left(1-\rho^{2}\right)+\sigma_{\omega}^{2}}$. The variable $\theta_{t}$ corresponds to abnormal expected returns ("Jensen alpha"), and its standard deviation in the cross-section of hedge funds examined in JMN is about $2.4 \%$ per year. JMN also report a cross-sectional standard deviation of fund returns $\left(s_{t}\right)$ ranging from $10 \%$ to $25 \%$ per year, depending on the year. Assuming that cross-sectional variances translate to unconditional ones in our model, the implied values of $\sigma_{\eta}^{2} / \sigma_{\omega}^{2}$ range from 0.0004 to 0.0025 . We also consider larger values, in line with Berk and Green's (2004) argument that the standard deviation of managerial ability can exceed that of fund returns. Finally, we use the calibration of Section 2 for the parameters of the gambler's fallacy, setting $\alpha=0.2$ and $\delta=0.7$. Table 2 reports Freddy's persistence estimate $\tilde{\rho}$, his trading volume relative to Tommy, and his relative value of information.

\begin{tabular}{|l|ccccc|}
\hline$\sigma_{\eta}^{2} / \sigma_{\omega}^{2}$ & 0.0004 & 0.001 & 0.0025 & 0.005 & 0.01 \\
\hline \hline Persistence Estimate $(\tilde{\rho})$ & 0.399 & 0.621 & 0.824 & 0.905 & 0.940 \\
\hline Rel. Volume $(\tilde{Q} / Q)$ & 1.728 & 1.834 & 1.635 & 1.254 & 0.916 \\
\hline Rel. Value Full Inf. $\left(\tilde{I}_{\infty} / I_{\infty}\right)$ & 0.238 & 0.450 & 0.774 & 0.921 & 0.966 \\
\hline Rel. Value $12-$ Month Inf. $\left(\tilde{I}_{12} / I_{12}\right)$ & 0.619 & 1.170 & 1.954 & 2.014 & 1.715 \\
\hline Rel. Value 6-Month Inf. $\left(\tilde{I}_{6} / I_{6}\right)$ & 1.104 & 2.049 & 2.754 & 2.197 & 1.648 \\
\hline
\end{tabular}

Table 2: Freddy's persistence estimate, relative volume, and relative value of information.

Consistent with Proposition 7, Freddy's persistence estimate $\tilde{\rho}$ increases with the extent of serial correlation, as measured by $\sigma_{\eta}^{2} / \sigma_{\omega}^{2}$. Freddy's trading volume exceeds Tommy's by more than $60 \%$ for the values of $\sigma_{\eta}^{2} / \sigma_{\omega}^{2}$ inferred by the data. For $\sigma_{\eta}^{2} / \sigma_{\omega}^{2}=0.01$ and larger values, Freddy's volume is below Tommy's but never by more than $11 \%$. The value of 
information that Freddy attributes to the full return history is below Tommy's for all values of $\sigma_{\eta}^{2} / \sigma_{\omega}^{2}$ in the table. This is especially so for smaller values because Freddy underestimates significantly the information content of distant returns. But consistent with Proposition 10, Freddy overestimates the content of recent returns. For all values of $\sigma_{\eta}^{2} / \sigma_{\omega}^{2}$ in the table, he would overpay to learn the six-month history, and for all but the smallest value he would overpay for the twelve-month history.

Imposing a prior belief that $\tilde{\rho} \geq \underline{\rho}>0$ would increase the ratios in the table. Suppose, for example, that $\underline{\rho}=0.6$, i.e., Freddy is confident that at least $60 \%$ of managerial ability in one month spills over to the next month. This would affect the column corresponding to $\sigma_{\eta}^{2} / \sigma_{\omega}^{2}=0.0004$ since in that case Freddy converges to $\tilde{\rho}=0.398<0.6$. The new persistence estimate becomes $\tilde{\rho}=0.6$, the relative volume jumps to 4.049 , and the relative values of information jump to $1.039,2.703$, and 4.749 , respectively. 


\section{A Proofs}

Proof of Proposition 1: Our formulation of the recursive-filtering problem is as in standard textbooks. For example, (9) and (10) follow from (4.1.1) and (4.1.4) in Balakrishnan (1987) if $x_{n+1}$ is replaced by $x_{t}, x_{n}$ by $x_{t-1}, A_{n}$ by $\tilde{A}, U_{n}$ by $0, N_{n}^{s}$ by $w_{t}, v_{n}$ by $s_{t}-\tilde{\mu}, C_{n}$ by $\tilde{C}$, and $N_{n}^{0}$ by $v_{t}$. Eq. (11) follows from (4.6.14), if the latter is written for $n+1$ instead of $n$, and $\bar{x}_{n+1}$ is replaced by $\bar{x}_{t}, \bar{x}_{n}$ by $\bar{x}_{t-1}$, and $A K_{n}+Q_{n}$ by $\tilde{G}_{t}$. That $\tilde{G}_{t}$ so defined is given by (13), follows from (4.1.29) and (4.6.12) if $H_{n-1}$ is replaced by $\tilde{\Sigma}_{t-1}, G_{n} G_{n}^{\prime}$ by $\tilde{V}$, and $J_{n}$ by $\tilde{U}$. Eq. (12) follows from (4.6.18) if the latter is written for $n+1$ instead of $n, P_{n}$ is substituted from (4.1.30), and $F_{n} F_{n}^{\prime}$ is replaced by $\tilde{W}$.

Proof of Proposition 2: It suffices to show (Balakrishnan, p.182-184) that the eigenvalues of $\tilde{A}-\tilde{U} \tilde{V}^{-1} \tilde{C}$ have modulus smaller than one. This matrix is

$$
\left[\begin{array}{cc}
\frac{\tilde{\sigma}_{\omega}^{2}}{\tilde{\sigma}_{\eta}^{2}+\tilde{\sigma}_{\omega}^{2}} \tilde{\rho} & \frac{\tilde{\sigma}_{\eta}^{2}}{\tilde{\sigma}_{\eta}^{2}+\tilde{\sigma}_{\omega}^{2}} \alpha_{\tilde{\rho}} \\
-\frac{\tilde{\sigma}_{\omega}^{2}}{\tilde{\sigma}_{\eta}^{2}+\tilde{\sigma}_{\omega}^{2}} \tilde{\rho} & \delta_{\tilde{\rho}}-\frac{\tilde{\sigma}_{\eta}^{2}}{\tilde{\sigma}_{\eta}^{2}+\tilde{\sigma}_{\omega}^{2}} \alpha_{\tilde{\rho}}
\end{array}\right]
$$

The characteristic polynomial is

$$
\begin{aligned}
& \lambda^{2}-\lambda\left[\frac{\tilde{\sigma}_{\omega}^{2}}{\tilde{\sigma}_{\eta}^{2}+\tilde{\sigma}_{\omega}^{2}} \tilde{\rho}+\delta_{\tilde{\rho}}-\frac{\tilde{\sigma}_{\eta}^{2}}{\tilde{\sigma}_{\eta}^{2}+\tilde{\sigma}_{\eta}^{2}} \alpha_{\tilde{\rho}}\right]+\frac{\tilde{\sigma}_{\omega}^{2}}{\tilde{\sigma}_{\eta}^{2}+\tilde{\sigma}_{\omega}^{2}} \tilde{\rho} \delta_{\tilde{\rho}} \\
\equiv & \lambda^{2}-\lambda b+c
\end{aligned}
$$

Suppose that the roots $\lambda_{1}, \lambda_{2}$ of this polynomial are real, in which case $\lambda_{1}+\lambda_{2}=b$ and $\lambda_{1} \lambda_{2}=c$. Since $c>0, \lambda_{1}$ and $\lambda_{2}$ have the same sign. If $\lambda_{1}$ and $\lambda_{2}$ are negative, they are both greater than -1 , since $b>-1$ from $\alpha_{\tilde{\rho}}<1$ and $\tilde{\rho}, \delta_{\tilde{\rho}} \geq 0$. If $\lambda_{1}$ and $\lambda_{2}$ are positive, then at least one is smaller than 1 , since $b<2$ from $\tilde{\rho}, \delta_{\tilde{\rho}}<1$ and $\alpha_{\tilde{\rho}} \geq 0$. But since the characteristic polynomial for $\lambda=1$ takes the value

$$
\left(1-\delta_{\tilde{\rho}}\right)\left(1-\frac{\tilde{\sigma}_{\omega}^{2}}{\tilde{\sigma}_{\eta}^{2}+\tilde{\sigma}_{\omega}^{2}} \tilde{\rho}\right)+\frac{\tilde{\sigma}_{\eta}^{2}}{\tilde{\sigma}_{\eta}^{2}+\tilde{\sigma}_{\omega}^{2}} \alpha_{\tilde{\rho}}>0
$$

both $\lambda_{1}$ and $\lambda_{2}$ are smaller than 1 . Suppose instead that $\lambda_{1}, \lambda_{2}$ are complex. In that case, they are conjugates and the modulus of each is $\sqrt{c}<1$.

Lemma A.1 determines $\bar{s}_{t}$, the true mean of $s_{t}$ conditional on $\mathcal{H}_{t-1}$, and $\bar{s}_{t}(\tilde{p})$, the mean that Freddy computes under the parameter vector $\tilde{p}$. To state the lemma, we define the 
matrices $\tilde{D}_{t} \equiv \tilde{A}-\tilde{G}_{t} \tilde{C}, \tilde{D} \equiv \tilde{A}-\tilde{G} \tilde{C}$, and

$$
\tilde{J}_{t, t^{\prime}} \equiv\left\{\begin{array}{cl}
\prod_{k=t^{\prime}}^{t} \tilde{D}_{k} & \text { for } t^{\prime}=1, . ., t \\
I & \text { for } t^{\prime}>t .
\end{array}\right.
$$

For simplicity, we set the initial condition $\bar{x}_{0}=0$.

Lemma A.1 The true mean $\bar{s}_{t}$ is given by

$$
\bar{s}_{t}=\mu+\sum_{t^{\prime}=1}^{t-1} C A^{t-t^{\prime}-1} G_{t^{\prime}} \zeta_{t^{\prime}}
$$

and Freddy's mean $\bar{s}_{t}(\tilde{p})$ by

$$
\bar{s}_{t}(\tilde{p})=\tilde{\mu}+\sum_{t^{\prime}=1}^{t-1} \tilde{C} \tilde{M}_{t, t^{\prime}} \zeta_{t^{\prime}}+\tilde{C} \tilde{M}_{t}^{\mu}(\mu-\tilde{\mu}),
$$

where

$$
\begin{gathered}
\tilde{M}_{t, t^{\prime}} \equiv \tilde{J}_{t-1, t^{\prime}+1} \tilde{G}_{t^{\prime}}+\sum_{k=t^{\prime}+1}^{t-1} \tilde{J}_{t-1, k+1} \tilde{G}_{k} C A^{k-t^{\prime}-1} G_{t^{\prime}} \\
\tilde{M}_{t}^{\mu} \equiv \sum_{t^{\prime}=1}^{t-1} \tilde{J}_{t-1, t^{\prime}+1} \tilde{G}_{t^{\prime}} .
\end{gathered}
$$

Proof: Consider the recursive-filtering problem under the true model, and denote by $\bar{x}_{t}$ the true mean of $x_{t}$. Eq. (10) implies that

$$
\bar{s}_{t}=\mu+C \bar{x}_{t-1} .
$$

Eq. (11) then implies that

$$
\bar{x}_{t}=A \bar{x}_{t-1}+G_{t}\left(s_{t}-\bar{s}_{t}\right)=A \bar{x}_{t-1}+G_{t} \zeta_{t} .
$$

Iterating between $t-1$ and zero, we find

$$
\bar{x}_{t-1}=\sum_{t^{\prime}=1}^{t-1} A^{t-t^{\prime}-1} G_{t^{\prime}} \zeta_{t^{\prime}} .
$$

Plugging into (A.3), we find (A.1). 
Consider next Freddy's recursive-filtering problem under $\tilde{p}$. Eq. (11) implies that

$$
\bar{x}_{t}(\tilde{p})=\left(\tilde{A}-\tilde{G}_{t} \tilde{C}\right) \bar{x}_{t-1}(\tilde{p})+\tilde{G}_{t}\left(s_{t}-\tilde{\mu}\right) .
$$

Iterating between $t-1$ and zero, we find

$$
\begin{aligned}
\bar{x}_{t-1}(\tilde{p}) & =\sum_{t^{\prime}=1}^{t-1} \tilde{J}_{t-1, t^{\prime}+1} \tilde{G}_{t^{\prime}}\left(s_{t^{\prime}}-\tilde{\mu}\right) \\
& =\sum_{t^{\prime}=1}^{t-1} \tilde{J}_{t-1, t^{\prime}+1} \tilde{G}_{t^{\prime}}\left(\zeta_{t^{\prime}}+\mu-\tilde{\mu}+C \bar{x}_{t^{\prime}-1}\right),
\end{aligned}
$$

where the second step follows from $s_{t^{\prime}}=\zeta_{t^{\prime}}+\bar{s}_{t^{\prime}}$ and (A.3). Substituting $\bar{x}_{t^{\prime}-1}$ from (A.4), and grouping terms, we find

$$
\bar{x}_{t-1}(\tilde{p})=\sum_{t^{\prime}=1}^{t-1} \tilde{M}_{t, t^{\prime}} \zeta_{t^{\prime}}+\tilde{M}_{t}^{\mu}(\mu-\tilde{\mu}) .
$$

Combining this with

$$
\bar{s}_{t}(\tilde{p})=\tilde{\mu}+\tilde{C} \bar{x}_{t-1}(\tilde{p})
$$

(which follows from (10)), we find (A.2).

We next prove Lemma 3. While this Lemma is stated after Theorem 1 and Lemmas 1 and 2 , its proof does not rely on these results.

Proof of Lemma 3: Lemma A.1 implies that

$$
\bar{s}_{t}(\tilde{p})-\bar{s}_{t}=\sum_{t^{\prime}=1}^{t-1} e_{t, t^{\prime}} \zeta_{t^{\prime}}+N_{t}^{\mu}(\tilde{\mu}-\mu),
$$

where

$$
\begin{gathered}
e_{t, t^{\prime}} \equiv \tilde{C} \tilde{M}_{t, t^{\prime}}-C A^{t-t^{\prime}-1} G_{t^{\prime}} \\
N_{t}^{\mu} \equiv 1-\tilde{C} \tilde{M}_{t}^{\mu}
\end{gathered}
$$

Therefore,

$$
\left[\bar{s}_{t}(p)-\bar{s}_{t}\right]^{2}=\sum_{t^{\prime}, t^{\prime \prime}=1}^{t-1} e_{t, t^{\prime}} e_{t, t^{\prime \prime}} \zeta_{t^{\prime}} \zeta_{t^{\prime \prime}}+\left(N_{t}^{\mu}\right)^{2}(\tilde{\mu}-\mu)^{2}+2 \sum_{t^{\prime}=1}^{t-1} e_{t, t^{\prime}} N_{t}^{\mu} \zeta_{t^{\prime}}(\tilde{\mu}-\mu) .
$$


Since the sequence $\left\{\zeta_{t^{\prime}}\right\}_{t^{\prime}=1, . ., t-1}$ is independent under the true measure and mean-zero, we have

$$
E\left[\bar{s}_{t}(p)-\bar{s}_{t}\right]^{2}=\sum_{t^{\prime}=1}^{t-1} e_{t, t^{\prime}}^{2} \sigma_{s, t^{\prime}}^{2}+\left(N_{t}^{\mu}\right)^{2}(\tilde{\mu}-\mu)^{2}
$$

We first determine the limit of $\sum_{t^{\prime}=1}^{t-1} e_{t, t^{\prime}}^{2} \sigma_{s, t^{\prime}}^{2}$ when $t$ goes to $\infty$. Defining the double sequence $\left\{\phi_{k, t}\right\}_{k, t \geq 1}$ by

$$
\phi_{k, t} \equiv\left\{\begin{array}{cc}
e_{t, t-k}^{2} \sigma_{s, t-k}^{2} & \text { for } k=1, . ., t-1, \\
0 & \text { for } k>t-1,
\end{array}\right.
$$

we have

$$
\sum_{t^{\prime}=1}^{t-1} e_{t, t^{\prime}}^{2} \sigma_{s, t^{\prime}}^{2}=\sum_{k=1}^{t-1} e_{t, t-k}^{2} \sigma_{s, t-k}^{2}=\sum_{k=1}^{\infty} \phi_{k, t}
$$

The definitions of $e_{t, t^{\prime}}$ and $\tilde{M}_{t, t^{\prime}}$ imply that

$$
e_{t, t-k}=\tilde{C} \tilde{J}_{t-1, t-k+1} \tilde{G}_{t-k}+\sum_{k^{\prime}=1}^{k-1} \tilde{C} \tilde{J}_{t-1, t-k+k^{\prime}+1} \tilde{G}_{t-k+k^{\prime}} C A^{k^{\prime}-1} G_{t-k}-C A^{k-1} G_{t-k}
$$

Eq. (10) applied to the recursive-filtering problem under the true model implies that

$$
\sigma_{s, t}^{2}=C \Sigma_{t-1} C^{\prime}+V
$$

When $t$ goes to $\infty, G_{t}$ goes to $G, \tilde{G}_{t}$ to $\tilde{G}, \Sigma_{t}$ to $\Sigma$, and $\tilde{J}_{t, t-k}$ to $\tilde{D}^{k+1}$. Therefore,

$$
\begin{gathered}
\lim _{t \rightarrow \infty} e_{t, t-k}=\tilde{C} \tilde{D}^{k-1} \tilde{G}+\sum_{k^{\prime}=1}^{k-1} \tilde{C} \tilde{D}^{k-1-k^{\prime}} \tilde{G} C A^{k^{\prime}-1} G-C A^{k-1} G=\tilde{N}_{k}-N_{k} \equiv e_{k}, \\
\lim _{t \rightarrow \infty} \sigma_{s, t-k}^{2}=C \Sigma C^{\prime}+V=C \Sigma C^{\prime}+\sigma_{\eta}^{2}+\sigma_{\omega}^{2}=\sigma_{s}^{2},
\end{gathered}
$$

implying that

$$
\lim _{t \rightarrow \infty} \phi_{k, t}=e_{k}^{2} \sigma_{s}^{2}
$$

The dominated convergence theorem will imply that

$$
\lim _{t \rightarrow \infty} \sum_{k=1}^{\infty} \phi_{k, t}=\sum_{k=1}^{\infty} \lim _{t \rightarrow \infty} \phi_{k, t}=\sigma_{s}^{2} \sum_{k=1}^{\infty} e_{k}^{2}
$$

if there exists a sequence $\left\{\bar{\phi}_{k}\right\}_{k \geq 1}$ such that $\sum_{k=1}^{\infty} \bar{\phi}_{k}<\infty$ and $\left|\phi_{k, t}\right| \leq \bar{\phi}_{k}$ for all $k, t \geq 1$. To 
construct such a sequence, we note that the eigenvalues of $A$ have modulus smaller than one, and so do the eigenvalues of $\tilde{D} \equiv \tilde{A}-\tilde{G} \tilde{C}$ (Balakrishnan, Theorem 4.2.3, p.111). Denoting by $a<1$ a number exceeding the maximum of the moduli, we can construct a dominating sequence $\left\{\bar{\phi}_{k}\right\}_{k \geq 1}$ decaying geometrically at the rate $a^{2 k}$.

We next determine the limit of $N_{t}^{\mu}$. Defining the double sequence $\left\{\chi_{k, t}\right\}_{k, t \geq 1}$ by

$$
\chi_{k, t} \equiv\left\{\begin{array}{cl}
\tilde{J}_{t-1, t-k+1} \tilde{G}_{t-k} & \text { for } k=1, . ., t-1 \\
0 & \text { for } k>t-1
\end{array}\right.
$$

we have

$$
N_{t}^{\mu}=1-\tilde{C} \sum_{k=1}^{t-1} \tilde{J}_{t-1, t-k+1} \tilde{G}_{t-k}=1-\tilde{C} \sum_{k=1}^{\infty} \chi_{k, t} .
$$

It is easy to check that the dominated convergence theorem applies to $\left\{\chi_{k, t}\right\}_{k, t \geq 1}$, and thus

$$
\lim _{t \rightarrow \infty} N_{t}^{\mu}=1-\tilde{C} \lim _{t \rightarrow \infty}\left[\sum_{k=1}^{\infty} \chi_{k, t}\right]=1-\tilde{C} \sum_{k=1}^{\infty} \lim _{t \rightarrow \infty} \chi_{k, t}=1-\tilde{C} \sum_{k=1}^{\infty} \tilde{D}^{k-1} \tilde{G}=N^{\mu}
$$

The lemma follows by combining (A.10), (A.13), and (A.14).

Proof of Theorem 1: Eq. (16) implies that

$$
\frac{2 \log L_{t}\left(\mathcal{H}_{t} \mid \tilde{p}\right)}{t}=-\frac{\sum_{t^{\prime}=1}^{t} \log \left[2 \pi \sigma_{s, t^{\prime}}^{2}(\tilde{p})\right]}{t}-\frac{1}{t} \sum_{t^{\prime}=1}^{t} \frac{\left[s_{t^{\prime}}-\bar{s}_{t^{\prime}}(\tilde{p})\right]^{2}}{\sigma_{s, t^{\prime}}^{2}(\tilde{p})}
$$

To determine the limit of the first term, we note that (10) applied to Freddy's recursivefiltering problem under $\tilde{p}$ implies that

$$
\sigma_{s, t}^{2}(\tilde{p})=\tilde{C} \tilde{\Sigma}_{t-1} \tilde{C}^{\prime}+\tilde{V}
$$

Therefore,

$$
\begin{gathered}
\lim _{t \rightarrow \infty} \sigma_{s, t}^{2}(\tilde{p})=\tilde{C} \tilde{\Sigma} \tilde{C}^{\prime}+\tilde{V}=\sigma_{s}^{2}(\tilde{p}) \\
\lim _{t \rightarrow \infty} \frac{\sum_{t^{\prime}=1}^{t} \log \sigma_{s, t^{\prime}}^{2}(\tilde{p})}{t}=\lim _{t \rightarrow \infty} \log \sigma_{s, t}^{2}(\tilde{p})=\log \sigma_{s}^{2}(\tilde{p})
\end{gathered}
$$


We next fix $k \geq 0$ and determine the limit of the sequence

$$
S_{k, t} \equiv \frac{1}{t} \sum_{t^{\prime}=1}^{t} \zeta_{t^{\prime}} \zeta_{t^{\prime}+k}
$$

when $t$ goes to $\infty$. This sequence involves averages of random variables that are nonindependent and non-identically distributed. An appropriate law of large numbers (LLN) for such sequences is that of McLeish (1975). Consider a probability space $(\Omega, \mathcal{F}, P)$, a sequence $\left\{\mathcal{F}_{t}\right\}_{t \in \mathbb{Z}}$ of $\sigma$-algebras, and a sequence $\left\{U_{t}\right\}_{t \geq 1}$ of random variables. The pair $\left(\left\{\mathcal{F}_{t}\right\}_{t \in \mathbb{Z}},\left\{U_{t}\right\}_{t \geq 1}\right)$ is a mixingale (McLeish, Definition 1.2, p.830) if and only if there exist sequences $\left\{c_{t}\right\}_{t \geq 1}$ and $\left\{\psi_{m}\right\}_{m \geq 0}$ of nonnegative constants, with $\lim _{m \rightarrow \infty} \psi_{m}=0$, such that for all $t \geq 1$ and $m \geq 0$ :

$$
\begin{gathered}
\left\|E_{t-m} U_{t}\right\|_{2} \leq \psi_{m} c_{t}, \\
\left\|U_{t}-E_{t+m} U_{t}\right\|_{2} \leq \psi_{m+1} c_{t},
\end{gathered}
$$

where $\|.\|_{2}$ denotes the $L_{2}$ norm, and $E_{t^{\prime}} U_{t}$ the expectation of $U_{t}$ conditional on $\mathcal{F}_{t^{\prime}}$. McLeish's LLN (Corollary 1.9, p.832) states that if $\left(\left\{\mathcal{F}_{t}\right\}_{t \in \mathbb{Z}},\left\{U_{t}\right\}_{t \geq 1}\right)$ is a mixingale, then

$$
\lim _{t \rightarrow \infty} \frac{1}{t} \sum_{t^{\prime}=1}^{t} U_{t^{\prime}}=0
$$

almost surely, provided that $\sum_{t=1}^{\infty} c_{t}^{2} / t^{2}<\infty$ and $\sum_{m=1}^{\infty} \psi_{m}<\infty$. In our model, we take the probability measure to be the true measure, and define the sequence $\left\{\mathcal{F}_{t}\right\}_{t \in \mathbb{Z}}$ as follows: $\mathcal{F}_{t}=\{\Omega, \emptyset\}$ for $t \leq 0$, and $\mathcal{F}_{t}$ is the $\sigma$-algebra generated by $\left\{\zeta_{t^{\prime}}\right\}_{t^{\prime}=1, . ., t}$ for $t \geq 1$. Moreover, we set $U_{t} \equiv \zeta_{t}^{2}-\sigma_{s, t}^{2}$ when $k=0$, and $U_{t} \equiv \zeta_{t} \zeta_{t+k}$ when $k \geq 1$. Since the sequence $\left\{\zeta_{t}\right\}_{t \geq 1}$ is independent, we have $E_{t-m} U_{t}=0$ for $m \geq 1$. We also trivially have $E_{t+m} U_{t}=U_{t}$ for $m \geq k$. Therefore, when $k=0,\left(\right.$ A.18) and (A.19) hold with $\psi_{0}=1, \psi_{m}=0$ for $m \geq 1$, and $c_{t}=\sup _{t \geq 1}\left\|\zeta_{t}^{2}-\sigma_{s, t}^{2}\right\|_{2}$ for $t \geq 1$. McLeish's LLN implies that

$$
\lim _{t \rightarrow \infty} S_{0, t}=\lim _{t \rightarrow \infty} \frac{1}{t} \sum_{t^{\prime}=1}^{t}\left(U_{t^{\prime}}+\sigma_{s, t^{\prime}}^{2}\right)=\lim _{t \rightarrow \infty} \frac{\sum_{t^{\prime}=1}^{t} \sigma_{s, t^{\prime}}^{2}}{t}=\lim _{t \rightarrow \infty} \sigma_{s, t}^{2}=\sigma_{s}^{2}
$$

almost surely. When $k \geq 1,(\mathrm{~A} .18)$ and (A.19) hold with $\psi_{m}=1$ for $m=0, . ., k-1, \psi_{m}=0$ 
for $m \geq k$, and $c_{t}=\sup _{t \geq 1}\left\|\zeta_{t}\right\|_{2}^{2}$ for $t \geq 1$. McLeish's LLN implies that

$$
\lim _{t \rightarrow \infty} S_{k, t}=\lim _{t \rightarrow \infty} \frac{1}{t} \sum_{t^{\prime}=1}^{t} U_{t^{\prime}}=0
$$

almost surely. Finally, a straightforward application of McLeish's LLN to the sequence $U_{t} \equiv \zeta_{t}$ implies that

$$
\lim _{t \rightarrow \infty} \frac{1}{t} \sum_{t^{\prime}=1}^{t} \zeta_{t^{\prime}}=0
$$

almost surely. Since $\mathbb{N}$ is countable, we can assume that (A.20), (A.21) for all $k \geq 1$, and (A.22), hold in the same measure-one set. In what follows, we consider histories in that set.

To determine the limit of the second term in (A.15), we write it as

$$
\underbrace{\frac{1}{t} \sum_{t^{\prime}=1}^{t} \frac{\zeta_{t^{\prime}}^{2}}{\sigma_{s, t^{\prime}}^{2}(\tilde{p})}}_{X_{t}}-\underbrace{\frac{2}{t} \sum_{t^{\prime}=1}^{t} \frac{\zeta_{t^{\prime}}\left[\bar{s}_{t^{\prime}}(\tilde{p})-\bar{s}_{t^{\prime}}\right]}{\sigma_{s, t^{\prime}}^{2}(\tilde{p})}}_{Y_{t}}+\underbrace{\frac{1}{t} \sum_{t^{\prime}=1}^{t} \frac{\left[\bar{s}_{t^{\prime}}(\tilde{p})-\bar{s}_{t^{\prime}}\right]^{2}}{\sigma_{s, t^{\prime}}^{2}(\tilde{p})}}_{Z_{t}} .
$$

Since $\lim _{t \rightarrow \infty} \sigma_{s, t}^{2}(\tilde{p})=\sigma_{s}^{2}(\tilde{p})$, we have

$$
\lim _{t \rightarrow \infty} X_{t}=\frac{1}{\sigma_{s}^{2}(\tilde{p})} \lim _{t \rightarrow \infty} \frac{1}{t} \sum_{t^{\prime}=1}^{t} \zeta_{t^{\prime}}^{2}=\frac{1}{\sigma_{s}^{2}(\tilde{p})} \lim _{t \rightarrow \infty} S_{0, t}=\frac{\sigma_{s}^{2}}{\sigma_{s}^{2}(\tilde{p})}
$$

Using (A.8), we can write $Y_{t}$ as

$$
Y_{t}=2 \sum_{k=1}^{\infty} \psi_{k, t}+\frac{2}{t} \sum_{t^{\prime}=1}^{t} \frac{N_{t^{\prime}}^{\mu}}{\sigma_{s, t^{\prime}}^{2}(\tilde{p})} \zeta_{t^{\prime}}(\tilde{\mu}-\mu)
$$

where the double sequence $\left\{\psi_{k, t}\right\}_{k, t \geq 1}$ is defined by

$$
\psi_{k, t} \equiv\left\{\begin{array}{cl}
\frac{1}{t} \sum_{t^{\prime}=k+1}^{t} \frac{e_{t^{\prime}, t^{\prime}-k}}{\sigma_{s, t^{\prime}}^{2}(\tilde{p})} \zeta_{t^{\prime}} \zeta_{t^{\prime}-k} & \text { for } k=1, . ., t-1 \\
0 & \text { for } k>t-1
\end{array}\right.
$$

Since $\lim _{t \rightarrow \infty} e_{t, t-k}=e_{k}$ and $\lim _{t \rightarrow \infty} N_{t}^{\mu}=N^{\mu}$, we have

$$
\lim _{t \rightarrow \infty} \psi_{k, t}=\frac{e_{k}}{\sigma_{s}^{2}(\tilde{p})} \lim _{t \rightarrow \infty} \frac{1}{t} \sum_{t^{\prime}=k+1}^{t} \zeta_{t^{\prime}} \zeta_{t^{\prime}-k}=\frac{e_{k}}{\sigma_{s}^{2}(\tilde{p})} \lim _{t \rightarrow \infty} S_{k, t}=0
$$




$$
\lim _{t \rightarrow \infty} \frac{1}{t} \sum_{t^{\prime}=1}^{t} \frac{N_{t^{\prime}}^{\mu}}{\sigma_{s, t^{\prime}}^{2}(\tilde{p})} \zeta_{t^{\prime}}(\tilde{\mu}-\mu)=\frac{N^{\mu}}{\sigma_{s}^{2}(\tilde{p})}(\tilde{\mu}-\mu) \lim _{t \rightarrow \infty} \frac{1}{t} \sum_{t^{\prime}=1}^{t} \zeta_{t^{\prime}}=0 .
$$

The dominated convergence theorem will imply that

$$
\lim _{t \rightarrow \infty} Y_{t}=2 \lim _{t \rightarrow \infty} \sum_{k=1}^{\infty} \psi_{k, t}=2 \sum_{k=1}^{\infty} \lim _{t \rightarrow \infty} \psi_{k, t}=0
$$

if there exists a sequence $\left\{\bar{\psi}_{k}\right\}_{k \geq 1}$ such that $\sum_{k=1}^{\infty} \bar{\psi}_{k}<\infty$ and $\left|\psi_{k, t}\right| \leq \bar{\psi}_{k}$ for all $k, t \geq 1$. Such a sequence can be constructed by the same argument as for $\phi_{k, t}$ (Lemma 3) since

$$
\left|\frac{1}{t} \sum_{t^{\prime}=k+1}^{t} \zeta_{t^{\prime}} \zeta_{t^{\prime}-k}\right| \leq \frac{1}{t} \sum_{t^{\prime}=k+1}^{t}\left|\zeta_{t^{\prime}}\right|\left|\zeta_{t^{\prime}-k}\right| \leq \sqrt{\frac{1}{t} \sum_{t^{\prime}=k+1}^{t} \zeta_{t^{\prime}}^{2} \sqrt{\frac{1}{t} \sum_{t^{\prime}=k+1}^{t} \zeta_{t^{\prime}-k}^{2}}} \leq \sup _{t \geq 1} S_{0, t}<\infty
$$

where the last inequality holds because the sequence $S_{0, t}$ is convergent.

Using similar arguments as for $Y_{t}$, we find

$$
\lim _{t \rightarrow \infty} Z_{t}=\frac{\sigma_{s}^{2}}{\sigma_{s}^{2}(\tilde{p})} \sum_{k=1}^{\infty} e_{k}^{2}+\frac{\left(N^{\mu}\right)^{2}}{\sigma_{s}^{2}(\tilde{p})}(\tilde{\mu}-\mu)^{2}=\frac{e(\tilde{p})}{\sigma_{s}^{2}(\tilde{p})}
$$

The theorem follows from (A.15), (A.17), (A.23), (A.24), and (A.25).

Proof of Lemma 1: We first show that the set $m(P)$ is non-empty. Denoting by $\tilde{\mu}_{0}$ the value that minimizes $(\tilde{\mu}-\mu)^{2}$ over the closed set $P_{\mu}$, we have

$$
F(\tilde{p}) \leq F\left(\tilde{\sigma}_{\eta}^{2}, \tilde{\rho}, \tilde{\sigma}_{\omega}^{2}, \tilde{\mu}_{0}\right) \equiv G\left(\tilde{\sigma}_{\eta}^{2}, \tilde{\rho}, \tilde{\sigma}_{\omega}^{2}\right)
$$

When $\left(\tilde{\sigma}_{\eta}^{2}, \tilde{\sigma}_{\omega}^{2}\right)$ go to zero, the covariance matrix $\tilde{\Sigma}$ goes to zero. ( $\tilde{\Sigma}$ is smaller than the covariance matrix when signals are not observable, and the latter matrix goes to zero.) Eq. (A.16) then implies that $\sigma_{s}^{2}(\tilde{p})$ goes to zero. Eq. (A.16) also implies that when $\tilde{\sigma}_{\eta}^{2}$ or $\tilde{\sigma}_{\omega}^{2}$ go to $\infty, \sigma_{s}^{2}(\tilde{p})$ goes to $\infty$. In both cases, $(17)$ implies that $G\left(\tilde{\sigma}_{\eta}^{2}, \tilde{\rho}, \tilde{\sigma}_{\omega}^{2}\right)$ goes to $-\infty$. Therefore, in maximizing $G\left(\tilde{\sigma}_{\eta}^{2}, \tilde{\rho}, \tilde{\sigma}_{\omega}^{2}\right)$ over the closed set $P_{\sigma, \rho}$, we can restrict ourselves to a compact subset in which $\left(\tilde{\sigma}_{\eta}^{2}, \tilde{\sigma}_{\omega}^{2}\right)$ are not both zero. Since the function $G\left(\tilde{\sigma}_{\eta}^{2}, \tilde{\rho}, \tilde{\sigma}_{\omega}^{2}\right)$ is continuous in such a subset, the set of its maximands is non-empty. The same is true for the set $m(P)$ of maximands of $F(\tilde{p})$.

To show that the measure $\pi_{t}$ converges weakly to a measure giving weight only to $m(P)$, it suffices to show that for all closed sets $S$ that have zero intersection with $m(P), \pi_{t}(S)$ goes 
to zero. (Billingsley, Theorem 29.1, p.390) Consider first the case where $S$ is bounded. Since $S$ is compact, the maximum $F_{S}$ of $F(\tilde{p})$ over $S$ is smaller than the value of $F(\tilde{p})$ in $m(P)$. Consider a compact neighborhood $B$ of a point in $m(P)$ such that the minimum $F_{B}$ of $F(\tilde{p})$ over $B$ exceeds $F_{S}$. Consider also two constants $\left(F_{1}, F_{2}\right)$ such that $F_{B}>F_{2}>F_{1}>F_{S}$. For large enough $t$,

$$
\min _{\tilde{p} \in B} \frac{\log L_{t}\left(\mathcal{H}_{t} \mid \tilde{p}\right)}{t}>F_{2} .
$$

Indeed, if (A.26) does not hold, there exists a convergent sequence $\left\{\tilde{p}_{t}\right\}_{t \geq 1}$ in $B$ such that

$$
\frac{\log L_{t}\left(\mathcal{H}_{t} \mid \tilde{p}_{t}\right)}{t} \leq F_{2}
$$

Denoting the limit of this sequence by $\tilde{p} \in B$, Theorem 1 implies that $F(\tilde{p}) \leq F_{2}$, a contradiction. (Theorem 1 concerns the convergence of the likelihood for a given $\tilde{p}$, but extending the argument to a convergent sequence $\left\{\tilde{p}_{t}\right\}_{t \geq 1}$ is straightforward.) Likewise, we can show that for large enough $t$,

$$
\max _{\tilde{p} \in S} \frac{\log L_{t}\left(\mathcal{H}_{t} \mid \tilde{p}\right)}{t}<F_{1}
$$

Bayes' law, (A.26), and (A.27) imply that for large enough $t$,

$$
\pi_{t}(S)=\frac{E_{\pi_{0}}\left[L_{t}\left(\mathcal{H}_{t} \mid \tilde{p}\right) 1_{\{\tilde{p} \in S\}}\right]}{E_{\pi_{0}}\left[L_{t}\left(\mathcal{H}_{t} \mid \tilde{p}\right)\right]}<\frac{E_{\pi_{0}}\left[L_{t}\left(\mathcal{H}_{t} \mid \tilde{p}\right) 1_{\{\tilde{p} \in S\}}\right]}{E_{\pi_{0}}\left[L_{t}\left(\mathcal{H}_{t} \mid \tilde{p}\right) 1_{\{\tilde{p} \in B\}}\right]}<\frac{\exp \left(t F_{1}\right) \pi_{0}(S)}{\exp \left(t F_{2}\right) \pi_{0}(B)}
$$

Since $F_{2}>F_{1}, \pi_{t}(S)$ goes to zero when $t$ goes to $\infty$. When $S$ is unbounded, we can consider an increasing sequence of bounded subsets.

Proof of Lemma 2: Consider $\tilde{p} \in P$ such that $e(\tilde{p})=e(P)$ and $\sigma_{s}^{2}(\tilde{p})=\sigma_{s}^{2}+e(\tilde{p})$. We will show that $F(\tilde{p}) \geq F(\hat{p})$ for any $\hat{p}=\left(\hat{\sigma}_{\eta}^{2}, \hat{\rho}, \hat{\sigma}_{\omega}^{2}, \hat{\mu}\right) \in P$. Denote by $\hat{\Sigma}$ and $\hat{G}$ the steady-state variance and regression coefficient for the recursive-filtering problem under $\hat{p}$, and by $\hat{\Sigma}_{\lambda}$ and $\hat{G}_{\lambda}$ those under $\hat{p}_{\lambda} \equiv\left(\lambda \hat{\sigma}_{\eta}^{2}, \hat{\rho}, \lambda \hat{\sigma}_{\omega}^{2}, \hat{\mu}\right)$ for $\lambda>0$. It is easy to check that $\lambda \hat{\Sigma}$ solves Equation (14) for $\hat{p}_{\lambda}$. Since this equation has a unique solution, $\hat{\Sigma}_{\lambda}=\lambda \hat{\Sigma}$. Equation (13) then implies that $\hat{G}_{\lambda}=\hat{G}$, and Equations (18) and (A.16) imply that $e\left(\hat{p}_{\lambda}\right)=e(\hat{p})$ and $\sigma_{s}^{2}\left(\hat{p}_{\lambda}\right)=\lambda \sigma_{s}^{2}(\hat{p})$. Therefore,

$$
F\left(\hat{p}_{\lambda}\right)=-\frac{1}{2}\left[\log \left[2 \pi \lambda \sigma_{s}^{2}(\hat{p})\right]+\frac{\sigma_{s}^{2}+e(\hat{p})}{\lambda \sigma_{s}^{2}(\hat{p})}\right] .
$$


Since this function is maximized for

$$
\lambda^{*}=\frac{\sigma_{s}^{2}+e(\hat{p})}{\sigma_{s}^{2}(\hat{p})}
$$

we have

$$
F(\hat{p}) \leq F\left(\hat{p}_{\lambda^{*}}\right)=-\frac{1}{2}\left[\log \left[2 \pi\left[\sigma_{s}^{2}+e(\hat{p})\right]\right]+1\right] \leq-\frac{1}{2}\left[\log \left[2 \pi\left[\sigma_{s}^{2}+e(\tilde{p})\right]\right]+1\right]=F(\tilde{p}) .
$$

The proof of the converse is along the same lines.

Lemma A.2 determines when a model can predict the signals equally well as the true model.

Lemma A.2 The error $e(\tilde{p})$ is zero if and only if

- $\tilde{C} \tilde{A}^{k-1} \tilde{G}=C A^{k-1} G$ for all $k \geq 1$

- $(1-\tilde{\rho})(\tilde{\mu}-\mu)=0$.

Proof: From Lemma 3 it suffices to show that $\left\{e_{k}\right\}_{k \geq 1}=0$ is equivalent to $\tilde{C} \tilde{A}^{k-1} \tilde{G}=$ $C A^{k-1} G$ for all $k \geq 1$, and $N^{\mu}(\tilde{\mu}-\mu)=0$ is equivalent to $(1-\tilde{\rho})(\tilde{\mu}-\mu)=0$. Setting $a_{k} \equiv \tilde{C} \tilde{A}^{k-1} \tilde{G}-C A^{k-1} G$ and

$$
b_{k} \equiv \tilde{D}^{k-1} \tilde{G}+\sum_{k^{\prime}=1}^{k-1} \tilde{D}^{k-1-k^{\prime}} \tilde{G} C A^{k^{\prime}-1} G-\tilde{A}^{k-1} \tilde{G}
$$

we have $e_{k}=\tilde{C} b_{k}+a_{k}$ for $k \geq 1$. Simple algebra shows that

$$
b_{k}=\tilde{D} b_{k-1}-\tilde{G} a_{k-1}
$$

Iterating between $k$ and one, and using the initial condition $b_{1}=0$, we find

$$
b_{k}=-\sum_{k^{\prime}=1}^{k-1} \tilde{D}^{k-1-k^{\prime}} \tilde{G} a_{k^{\prime}}
$$

Therefore,

$$
e_{k}=-\sum_{k^{\prime}=1}^{k-1} \tilde{C} \tilde{D}^{k-1-k^{\prime}} \tilde{G} a_{k^{\prime}}+a_{k}
$$


Eq. (A.29) implies that $\left\{e_{k}\right\}_{k \geq 1}=0$ if and only if $\left\{a_{k}\right\}_{k \geq 1}=0$.

Eq. (21) implies that

$$
N^{\mu}=1-\tilde{C}(I-\tilde{D})^{-1} \tilde{G}=1-\tilde{C}(I-\tilde{A}+\tilde{G} \tilde{C})^{-1} \tilde{G} .
$$

Replacing $\tilde{A}$ and $\tilde{C}$ by their values, and denoting the components of $\tilde{G}$ by $\tilde{G}_{1}$ and $\tilde{G}_{2}$, we find

$$
N^{\mu}=1-\tilde{C}(I-\tilde{A}+\tilde{G} \tilde{C})^{-1} \tilde{G}=\frac{(1-\tilde{\rho})\left(1-\delta_{\tilde{\rho}}+\alpha_{\tilde{\rho}}\right)}{\left[1-\tilde{\rho}\left(1-\tilde{G}_{1}\right)\right]\left(1-\delta_{\tilde{\rho}}+\alpha_{\tilde{\rho}}\right)-\alpha_{\tilde{\rho}}(1-\tilde{\rho}) \tilde{G}_{2}} .
$$

Since $\alpha_{\tilde{\rho}}, \delta_{\tilde{\rho}} \in[0,1), N^{\mu}(\tilde{\mu}-\mu)=0$ is equivalent to $(1-\tilde{\rho})(\tilde{\mu}-\mu)=0$.

Proof of Proposition 3: Tommy can achieve minimum error $e\left(P_{0}\right)=0$ by using the vector of true parameters $p$. Since $e\left(P_{0}\right)=0$, Lemmas 2 and A.2 imply that $\tilde{p} \in m\left(P_{0}\right)$ if and only if (i) $\tilde{C} \tilde{A}^{k-1} \tilde{G}=C A^{k-1} G$ for all $k \geq 1$, (ii) $(1-\tilde{\rho})(\tilde{\mu}-\mu)=0$, and (iii) $\sigma_{s}^{2}(\tilde{p})=\sigma_{s}^{2}$. Since $\alpha=0$ for Tommy, we can write Condition (i) as

$$
\tilde{\rho}^{k} \tilde{G}_{1}=\rho^{k} G_{1}
$$

We can also write element $(1,1)$ of $(14)$ as

$$
\begin{aligned}
& \tilde{\Sigma}_{11}=\frac{\left(\tilde{\rho}^{2} \tilde{\Sigma}_{11}+\tilde{\sigma}_{\eta}^{2}\right) \tilde{\sigma}_{\omega}^{2}}{\tilde{\rho}^{2} \tilde{\Sigma}_{11}+\tilde{\sigma}_{\eta}^{2}+\tilde{\sigma}_{\omega}^{2}}, \\
& \Sigma_{11}=\frac{\left(\rho^{2} \Sigma_{11}+\sigma_{\eta}^{2}\right) \sigma_{\omega}^{2}}{\rho^{2} \Sigma_{11}+\sigma_{\eta}^{2}+\sigma_{\omega}^{2}},
\end{aligned}
$$

and the first element of (15) as

$$
\begin{aligned}
\tilde{G}_{1} & =\frac{\tilde{\rho}^{2} \tilde{\Sigma}_{11}+\tilde{\sigma}_{\eta}^{2}}{\tilde{\rho}^{2} \tilde{\Sigma}_{11}+\tilde{\sigma}_{\eta}^{2}+\tilde{\sigma}_{\omega}^{2}}, \\
G_{1} & =\frac{\rho^{2} \Sigma_{11}+\sigma_{\eta}^{2}}{\rho^{2} \Sigma_{11}+\sigma_{\eta}^{2}+\sigma_{\omega}^{2}},
\end{aligned}
$$

where the first equation in each case is for $\tilde{p}$ and the second for $p$. Using (A.12) and (A.16), we can write Condition (iii) as

$$
\tilde{\rho}^{2} \tilde{\Sigma}_{11}+\tilde{\sigma}_{\eta}^{2}+\tilde{\sigma}_{\omega}^{2}=\rho^{2} \Sigma_{11}+\sigma_{\eta}^{2}+\sigma_{\omega}^{2}
$$


Suppose that $\rho \sigma_{\eta}^{2}>0$, and consider $\tilde{p}$ that satisfies Conditions (i)-(iii). Eq. (A.35) implies that $G_{1}>0$. Since (A.31) must hold for all $k \geq 1$, we have $\tilde{\rho}=\rho$ and $\tilde{G}_{1}=G_{1}$. We next write (A.32)-(A.35) in terms of the normalized variables $\tilde{s}_{\eta}^{2} \equiv \tilde{\sigma}_{\eta}^{2} / \tilde{\sigma}_{\omega}^{2}, \tilde{S}_{11} \equiv \tilde{\Sigma}_{11} / \tilde{\sigma}_{\omega}^{2}, s_{\eta}^{2} \equiv \sigma_{\eta}^{2} / \sigma_{\omega}^{2}$, and $S_{11} \equiv \Sigma_{11} / \sigma_{\omega}^{2}$. Eqs. (A.32) and (A.33) imply that $\tilde{S}_{11}=g\left(\tilde{s}_{\eta}^{2}\right)$ and $S_{11}=g\left(s_{\eta}^{2}\right)$ for the same function $g$. Eqs. (A.34), (A.35), and $\tilde{G}_{1}=G_{1}$ then imply that $\tilde{s}_{\eta}^{2}=s_{\eta}^{2}$, and (A.36) implies that $\tilde{\sigma}_{\omega}^{2}=\sigma_{\omega}^{2}$. Since $\tilde{\rho}<1$, Condition (ii) implies that $\tilde{\mu}=\mu$. Thus, $\tilde{p}=p$.

Suppose next that $\rho \sigma_{\eta}^{2}=0$, and consider $\tilde{p}$ that satisfies Conditions (i)-(iii). If $\rho=0$, (A.31) implies that $\tilde{\rho}^{k} \tilde{G}_{1}=0$, and (A.36) that $\tilde{\rho}^{2} \tilde{\Sigma}_{11}+\tilde{\sigma}_{\eta}^{2}+\tilde{\sigma}_{\omega}^{2}=\sigma_{\eta}^{2}+\sigma_{\omega}^{2}$. If $\sigma_{\eta}^{2}=0$, the same implications follow because $\Sigma=0$ and $G=[0,1]^{\prime}$ from (14) and (15). Eq. $\tilde{\rho}^{k} \tilde{G}_{1}=0$ implies that either $\tilde{\rho}=0$, or $\tilde{G}_{1}=0$ in which case $\tilde{\sigma}_{\eta}^{2}=0$. If $\tilde{\rho}=0$, then $\tilde{\sigma}_{\eta}^{2}+\tilde{\sigma}_{\omega}^{2}=\sigma_{\eta}^{2}+\sigma_{\omega}^{2}$ and $\tilde{\mu}=\mu$. If $\tilde{\sigma}_{\eta}^{2}=0$, then $\tilde{\sigma}_{\omega}^{2}=\sigma_{\eta}^{2}+\sigma_{\omega}^{2}$, and if in addition $\tilde{\rho}<1$, then $\tilde{\mu}=\mu$. Therefore, $\tilde{p}$ is as in the proposition. Showing that all $\tilde{p}$ in the proposition satisfy Conditions (i)-(iii) is obvious.

Proof of Proposition 4: We determine the parameter vectors $\tilde{p}$ that belong to $m\left(P_{0}\right)$ and satisfy $e(\tilde{p})=0$. From Lemmas 2 and A.2, these must satisfy (i) $\tilde{C} \tilde{A}^{k-1} \tilde{G}=C A^{k-1} G$ for all $k \geq 1$, (ii) $(1-\tilde{\rho})(\tilde{\mu}-\mu)=0$, and (iii) $\sigma_{s}^{2}(\tilde{p})=\sigma_{s}^{2}$. Since $\rho \sigma_{\eta}^{2}=0$, we can write Condition (i) as

$$
\tilde{\rho}^{k} \tilde{G}_{1}-\alpha_{\tilde{\rho}}\left(\delta_{\tilde{\rho}}-\alpha_{\tilde{\rho}}\right)^{k-1} \tilde{G}_{2}=0
$$

and Condition (iii) as

$$
\tilde{C} \tilde{\Sigma} \tilde{C}^{\prime}+\tilde{V}=\sigma_{\eta}^{2}+\sigma_{\omega}^{2}
$$

Using the definitions of $\left(\alpha_{\tilde{\rho}}, \delta_{\tilde{\rho}}\right)$, we can write $(\mathrm{A} .37)$ as

$$
\tilde{\rho}^{k}\left[\tilde{G}_{1}-\alpha(\delta-\alpha)^{k-1} \tilde{G}_{2}\right]=0
$$

If $\tilde{\rho} \neq 0$, (A.39) implies that $\tilde{G}_{1}-\alpha(\delta-\alpha)^{k-1} \tilde{G}_{2}=0$ for all $k \geq 1$, which in turn implies that $\tilde{G}=0$. For $\tilde{G}=0,(14)$ becomes $\tilde{\Sigma}=\tilde{A} \tilde{\Sigma} \tilde{A}^{\prime}+\tilde{W}$. Solving for $\tilde{\Sigma}$, we find

$$
\begin{aligned}
& \tilde{\Sigma}_{11}=\frac{\tilde{\sigma}_{\eta}^{2}}{1-\tilde{\rho}^{2}}, \\
& \tilde{\Sigma}_{12}=0, \\
& \tilde{\Sigma}_{22}=\frac{\tilde{\sigma}_{\omega}^{2}}{1-\left(\delta_{\tilde{\rho}}-\alpha_{\tilde{\rho}}\right)^{2}} .
\end{aligned}
$$


Substituting $\tilde{\Sigma}$ into (15), we find $\left(\tilde{\sigma}_{\eta}^{2}, \tilde{\sigma}_{\omega}^{2}\right)=0$. But then, $\tilde{\Sigma}=0$, which contradicts (A.38) since $\sigma_{\omega}^{2}=\sigma_{\epsilon}^{2}>0$. Therefore, $\tilde{\rho}=0$. Eq. (A.38) then implies that $\tilde{\sigma}_{\eta}^{2}+\tilde{\sigma}_{\omega}^{2}=\sigma_{\eta}^{2}+\sigma_{\omega}^{2}$, and Condition (ii) implies that $\tilde{\mu}=\mu$. Showing that all $\tilde{p}$ in the proposition satisfy Conditions (i)-(iii) is obvious.

Proof of Proposition 5: Consider a sequence $\left\{\alpha_{n}\right\}_{n \in \mathbb{N}}$ converging to zero, and an element $\tilde{p}_{n} \equiv\left(\left(\tilde{\sigma}_{\eta}^{2}\right)_{n}, \tilde{\rho}_{n},\left(\tilde{\sigma}_{\omega}^{2}\right)_{n}, \tilde{\mu}_{n}\right)$ from the set $m\left(P_{\underline{\rho}}\right)$ corresponding to $\alpha_{n}$. The proposition will follow if we show that $\left(\frac{\left(\tilde{s}_{\eta}^{2}\right)_{n}}{\alpha_{n}}, \tilde{\rho}_{n},\left(\tilde{\sigma}_{\omega}^{2}\right)_{n}, \tilde{\mu}_{n}\right)$ converges to $\left(z, \underline{\rho}, \sigma_{\omega}^{2}, \mu\right)$, where $\left(\tilde{s}_{\eta}^{2}\right)_{n} \equiv\left(\tilde{\sigma}_{\eta}^{2}\right)_{n} /\left(\tilde{\sigma}_{\omega}^{2}\right)_{n}$. Denoting the limits of $\left(\frac{\left(\tilde{s}_{\eta}^{2}\right)_{n}}{\alpha_{n}},\left(\tilde{\sigma}_{\eta}^{2}\right)_{n}, \tilde{\rho}_{n},\left(\tilde{\sigma}_{\omega}^{2}\right)_{n}, \tilde{\mu}_{n}\right)$ by $\left(\ell_{s}, \ell_{\eta}, \ell_{\rho}, \ell_{\omega}, \ell_{\mu}\right)$, the point $\left(\ell_{\eta}, \ell_{\rho}, \ell_{\omega}, \ell_{\mu}\right)$ belongs to the set $m\left(P_{\rho}\right)$ derived for $\alpha=\sigma_{\eta}^{2}=0$. (If the sequences do not converge, we extract converging subsequences.) Since all elements in that set satisfy $\tilde{\rho} \geq \underline{\rho}>0$, Proposition 3 implies that they also satisfy $\tilde{\sigma}_{\eta}^{2}=0$ and $\tilde{\sigma}_{\omega}^{2}=\sigma_{\omega}^{2}$. Therefore, $\ell_{\eta}=0$ and $\ell_{\omega}=\sigma_{\omega}^{2}$.

We next determine $\left(\ell_{s}, \ell_{\rho}, \ell_{\mu}\right)$ under the assumption $\ell_{\rho}<1$. When $v \equiv\left(\alpha, \frac{\tilde{s}_{\eta}^{2}}{\alpha}, \tilde{\sigma}_{\eta}^{2}, \tilde{\rho}, \tilde{\sigma}_{\omega}^{2}\right)$ converges to $\ell_{v} \equiv\left(0, \ell_{s}, 0, \ell_{\rho}, \sigma_{\omega}^{2}\right), \tilde{C} \tilde{D}^{k} \tilde{G}$ converges to zero, $\frac{\tilde{G}_{1}}{\tilde{s}_{\eta}^{2}}$ to $\frac{1}{1-\ell_{\rho}^{2}}$, and $\tilde{G}_{2}$ to 1 . These limits follow by continuity if we show that when $\alpha=\tilde{\sigma}_{\eta}^{2}=0, \tilde{C} \tilde{D}^{k} \tilde{G}=0$ and $\tilde{G}_{2}=1$, and when $\alpha=0, \lim _{\tilde{\sigma}_{\eta}^{2} \rightarrow 0} \frac{\tilde{G}_{1}}{\tilde{s}_{\eta}^{2}}=\frac{1}{1-\tilde{\rho}^{2}}$. When $\tilde{\sigma}_{\eta}^{2}=0$, the unique solution of $(14)$ is $\tilde{\Sigma}=0$, and (15) implies that $\tilde{G}=[0,1]^{\prime}$. Therefore, when $\alpha=\tilde{\sigma}_{\eta}^{2}=0$, we have $\tilde{C} \tilde{G}=0$,

$$
\tilde{C} \tilde{D}=\tilde{C}(\tilde{A}-\tilde{G} \tilde{C})=\tilde{C} \tilde{A}=\tilde{\rho} \tilde{C}
$$

and $\tilde{C} \tilde{D}^{k} \tilde{G}=\tilde{\rho}^{k} \tilde{C} \tilde{G}=0$. Moreover, if we divide both sides of (A.32) and (A.34) (derived from (14) and (15) when $\alpha=0$ ) by $\tilde{\sigma}_{\omega}^{2}$, we find

$$
\begin{aligned}
\tilde{S}_{11} & =\frac{\left(\tilde{\rho}^{2} \tilde{S}_{11}+\tilde{s}_{\eta}^{2}\right)}{\tilde{\rho}^{2} \tilde{S}_{11}+\tilde{s}_{\eta}^{2}+1} \\
\tilde{G}_{1} & =\frac{\tilde{\rho}^{2} \tilde{S}_{11}+\tilde{s}_{\eta}^{2}}{\tilde{\rho}^{2} \tilde{S}_{11}+\tilde{s}_{\eta}^{2}+1} .
\end{aligned}
$$

When $\tilde{\sigma}_{\eta}^{2}$ converges to zero, $\tilde{s}_{\eta}^{2}$ and $\tilde{S}_{11}$ converge to zero. Eqs. (A.40) and (A.41) then imply that $\frac{\tilde{S}_{11}}{\tilde{s}_{\eta}^{2}}$ and $\frac{\tilde{G}_{1}}{\tilde{s}_{\eta}^{2}}$ converge to $\frac{1}{1-\tilde{\rho}^{2}}$. 
Using the above limits, we find

$$
\begin{aligned}
& \lim _{v \rightarrow \ell_{v}} \frac{\tilde{C} \tilde{A}^{k-1} \tilde{G}}{\alpha}=\lim _{v \rightarrow \ell_{v}} \frac{\tilde{\rho}^{k} \tilde{G}_{1}-\alpha_{\tilde{\rho}}\left(\delta_{\tilde{\rho}}-\alpha_{\tilde{\rho}}\right)^{k-1} \tilde{G}_{2}}{\alpha} \\
= & \lim _{v \rightarrow \ell_{v}} \tilde{\rho}^{k}\left[\frac{\tilde{s}_{\eta}^{2}}{\alpha} \frac{\tilde{G}_{1}}{\tilde{s}_{\eta}^{2}}-(\delta-\alpha)^{k-1} \tilde{G}_{2}\right]=\ell_{\rho}^{k}\left(\frac{\ell_{s}}{1-\ell_{\rho}^{2}}-\delta^{k-1}\right) .
\end{aligned}
$$

Eqs. (19), (A.29), $\lim _{v \rightarrow \ell_{v}} \tilde{C} \tilde{D}^{k} \tilde{G}=0$, and $N_{k}=C A^{k} G=0$, imply that

$$
\lim _{v \rightarrow \ell_{v}} \frac{e_{k}}{\alpha}=\lim _{v \rightarrow \ell_{v}} \frac{\tilde{N}_{k}}{\alpha}=\lim _{v \rightarrow \ell_{v}} \frac{\tilde{C} \tilde{D}^{k-1} \tilde{G}}{\alpha}=\lim _{v \rightarrow \ell_{v}} \frac{a_{k}}{\alpha}=\lim _{v \rightarrow \ell_{v}} \frac{\tilde{C} \tilde{A}^{k-1} \tilde{G}}{\alpha}=\ell_{\rho}^{k}\left(\frac{\ell_{s}}{1-\ell_{\rho}^{2}}-\delta^{k-1}\right) .
$$

Since $\tilde{p}_{n}$ minimizes $e\left(\tilde{p}_{n}\right),(18)$ implies that $\left(\left(\sigma_{\eta}^{2}\right)_{n}, \tilde{\rho}_{n},\left(\sigma_{\omega}^{2}\right)_{n}\right)$ minimizes $\sigma_{s}^{2}(\tilde{p}) \sum_{k=0}^{\infty} e_{k}^{2}$. Since from (A.42),

$$
\lim _{v \rightarrow \ell_{v}} \frac{\sigma_{s}^{2}(\tilde{p}) \sum_{k=1}^{\infty} e_{k}^{2}}{\alpha^{2}}=\sigma_{\omega}^{2} \sum_{k=1}^{\infty} \ell_{\rho}^{2 k}\left(\frac{\ell_{s}}{1-\ell_{\rho}^{2}}-\delta^{k-1}\right)^{2} \equiv \sigma_{\omega}^{2} F\left(\ell_{s}, \ell_{\rho}\right)
$$

$\left(\ell_{s}, \ell_{\rho}\right)$ must minimize $F$. Treating $F$ as a function of $\left(\ell_{s} /\left(1-\ell_{\rho}^{2}\right), \ell_{\rho}\right)$, the minimizing value of the second argument is clearly $\ell_{\rho}=\underline{\rho}$. The first-order condition w.r.t. the first argument is

$$
\sum_{k=1}^{\infty} \ell_{\rho}^{2 k}\left(\frac{\ell_{s}}{1-\ell_{\rho}^{2}}-\delta^{k-1}\right)=0
$$

and implies $\ell_{s}=z$. Finally, $\ell_{\mu}=\mu$ since $\left(\ell_{\eta}, \ell_{\rho}, \ell_{\omega}, \ell_{\mu}\right)$ belongs to the set $m\left(P_{\underline{\rho}}\right)$ derived for $\alpha=\sigma_{\eta}^{2}=0$, and $\ell_{\rho}<1$.

To show that $\ell_{\rho}$ cannot equal one, we proceed by contradiction. Denoting the limit of $\left(\tilde{G}_{1}\right)_{n} / \alpha_{n}$ by $\ell_{G}$, and setting $\hat{v} \equiv\left(\alpha, \frac{\tilde{G}_{1}}{\alpha}, \tilde{\sigma}_{\eta}^{2}, \tilde{\rho}, \tilde{\sigma}_{\omega}^{2}\right)$ and $\ell_{\hat{v}} \equiv\left(0, \ell_{G}, 0,1, \sigma_{\omega}^{2}\right)$, we have

$$
\lim _{\hat{v} \rightarrow \ell_{\hat{v}}} \frac{\sigma_{s}^{2}(\tilde{p}) \sum_{k=1}^{\infty} e_{k}^{2}}{\alpha^{2}}=\sigma_{\omega}^{2} \sum_{k=1}^{\infty}\left(\ell_{G}-\delta^{k-1}\right)^{2}=\sigma_{\omega}^{2} \lim _{\ell_{\rho} \rightarrow 1} F\left(\ell_{G}\left(1-\ell_{\rho}^{2}\right), \ell_{\rho}\right)>\sigma_{\omega}^{2} F(z, r)
$$

a contradiction because $\tilde{p}_{n}$ minimizes $e\left(\tilde{p}_{n}\right)$.

Proof of Proposition 6: Eqs. (A.5) and (A.7) imply that in steady state

$$
\tilde{E}_{t-1}\left(s_{t}\right) \equiv \bar{s}_{t}(\tilde{p})=\tilde{\mu}+\sum_{k=1}^{\infty} \tilde{C} \tilde{D}^{k-1} \tilde{G}\left(s_{t-k}-\tilde{\mu}\right) .
$$


Therefore,

$$
\sum_{k^{\prime}=1}^{k} \frac{\partial \tilde{E}_{t-1}\left(s_{t}\right)}{\partial s_{t-k^{\prime}}}=\sum_{k^{\prime}=1}^{k} \tilde{C} \tilde{D}^{k^{\prime}-1} \tilde{G}
$$

Eq. (A.42) implies that

$$
\sum_{k^{\prime}=1}^{k} \frac{\partial \tilde{E}_{t-1}\left(s_{t}\right)}{\partial s_{t-k^{\prime}}}=\alpha g_{k}+o(\alpha)
$$

where

$$
\begin{aligned}
g_{k} & \equiv \sum_{k^{\prime}=1}^{k} f_{k^{\prime}}, \\
f_{k} & \equiv \underline{\rho}^{k}\left(\frac{z}{1-\underline{\rho}^{2}}-\delta^{k-1}\right) .
\end{aligned}
$$

Using (25) to substitute $z$, we find

$$
\begin{gathered}
f_{1}=g_{1}=\frac{\underline{\rho}^{3}(\delta-1)}{1-\underline{\rho}^{2} \delta}<0, \\
g_{\infty}=\underline{\rho}\left[\frac{z}{\left(1-\underline{\rho}^{2}\right)(1-\underline{\rho})}-\frac{1}{1-\underline{\rho} \delta}\right]=\frac{\underline{\rho}^{2}(1-\delta)}{\left(1-\underline{\rho}^{2} \delta\right)(1-\underline{\rho} \delta)}>0 .
\end{gathered}
$$

The function $f_{k}$ is negative for $k=1$ and positive for large $k$. Since it can change sign only once, it is negative and then positive. The function $g_{k}$ is negative for $k=1$, then decreases $\left(f_{k}<0\right)$, then increases $\left(f_{k}>0\right)$, and is eventually positive $\left(g_{\infty}>0\right)$. Therefore, $g_{k}$ is negative and then positive.

Proof of Proposition 7: Consider a sequence $\left\{\alpha_{n}\right\}_{n \in \mathbb{N}}$ converging to zero, an element $\tilde{p}_{n} \equiv\left(\left(\tilde{\sigma}_{\eta}^{2}\right)_{n}, \tilde{\rho}_{n},\left(\tilde{\sigma}_{\omega}^{2}\right)_{n}, \tilde{\mu}_{n}\right)$ from the set $m\left(P_{0}\right)$ corresponding to $\alpha_{n}$, and set $\left(\sigma_{\eta}^{2}\right)_{n} \equiv \nu \alpha_{n}$. The proposition will follow if we show that $\left(\frac{\left(\tilde{s}_{\eta}^{2}\right)_{n}}{\alpha_{n}}, \tilde{\rho}_{n},\left(\tilde{\sigma}_{\omega}^{2}\right)_{n}, \tilde{\mu}_{n}\right)$ converges to $\left(z, r, \sigma_{\omega}^{2}, \mu\right)$. Denoting the limits of $\left(\frac{\left(\tilde{s}_{\eta}^{2}\right)_{n}}{\alpha_{n}},\left(\tilde{\sigma}_{\eta}^{2}\right)_{n}, \tilde{\rho}_{n},\left(\tilde{\sigma}_{\omega}^{2}\right)_{n}, \tilde{\mu}_{n}\right)$ by $\left(\ell_{s}, \ell_{\eta}, \ell_{\rho}, \ell_{\omega}, \ell_{\mu}\right)$, the point $\left(\ell_{\eta}, \ell_{\rho}, \ell_{\omega}, \ell_{\mu}\right)$ belongs to the set $m\left(P_{0}\right)$ derived for $\alpha=\sigma_{\eta}^{2}=0$.

Suppose that $\ell_{\rho} \in(0,1)$. Proposition 3 implies that $\ell_{\eta}=0, \ell_{\omega}=\sigma_{\omega}^{2}$, and $\ell_{\mu}=\mu$. The same argument as in the proof of Proposition 5 implies that $\left(\ell_{s}, \ell_{\rho}\right)$ must minimize the function

$$
H\left(\ell_{s}, \ell_{\rho}\right) \equiv \sum_{k=1}^{\infty}\left[\ell_{\rho}^{k}\left(\frac{\ell_{s}}{1-\ell_{\rho}^{2}}-\delta^{k-1}\right)-\rho^{k} \frac{\nu}{1-\rho^{2}}\right]^{2} .
$$

Treating $H$ as a function of $\left(\ell_{\rho}, \ell_{s} /\left(1-\ell_{\rho}^{2}\right)\right)$, the first-order condition w.r.t. the first argument 
is

$$
\sum_{k=1}^{\infty} \ell_{\rho}^{k}\left[\ell_{\rho}^{k}\left(\frac{\ell_{s}}{1-\ell_{\rho}^{2}}-\delta^{k-1}\right)-\rho^{k} \frac{\nu}{1-\rho^{2}}\right]=0
$$

and w.r.t. the second is

$$
\sum_{k=1}^{\infty} k \ell_{\rho}^{k-1}\left(\frac{\ell_{s}}{1-\ell_{\rho}^{2}}-\delta^{k-1}\right)\left[\ell_{\rho}^{k}\left(\frac{\ell_{s}}{1-\ell_{\rho}^{2}}-\delta^{k-1}\right)-\rho^{k} \frac{\nu}{1-\rho^{2}}\right]=0
$$

Computing the infinite sums, we can write (A.43) as

$$
\frac{\ell_{s} \ell_{\rho}}{\left(1-\ell_{\rho}^{2}\right)^{2}}-\frac{\ell_{\rho}}{1-\ell_{\rho}^{2} \delta}-\frac{\nu \rho}{\left(1-\rho^{2}\right)\left(1-\rho \ell_{\rho}\right)}=0
$$

and (A.44) as

$$
\begin{aligned}
& \frac{\ell_{s}}{1-\ell_{\rho}^{2}}\left[\frac{\ell_{s} \ell_{\rho}}{\left(1-\ell_{\rho}^{2}\right)^{3}}-\frac{\ell_{\rho}}{\left(1-\ell_{\rho}^{2} \delta\right)^{2}}-\frac{\nu \rho}{\left(1-\rho^{2}\right)\left(1-\rho \ell_{\rho}\right)^{2}}\right] \\
& -\left[\frac{\ell_{s} \ell_{\rho}}{\left(1-\ell_{\rho}^{2}\right)\left(1-\ell_{\rho}^{2} \delta\right)^{2}}-\frac{\ell_{\rho}}{\left(1-\ell_{\rho}^{2} \delta^{2}\right)^{2}}-\frac{\nu \rho}{\left(1-\rho^{2}\right)\left(1-\rho \ell_{\rho} \delta\right)^{2}}\right]=0 .
\end{aligned}
$$

Substituting $\ell_{s}$ from (A.45), we can write the first square bracket in (A.46) as

$$
-\frac{\nu \rho \ell_{\rho}\left(\rho-\ell_{\rho}\right)}{\left(1-\rho^{2}\right)\left(1-\rho \ell_{\rho}\right)^{2}\left(1-\ell_{\rho}^{2}\right)}+\frac{\ell_{\rho}^{3}(1-\delta)}{\left(1-\ell_{\rho}^{2}\right)\left(1-\ell_{\rho}^{2} \delta\right)}
$$

and the second as

$$
\frac{\nu \rho \ell_{\rho}\left(\rho-\ell_{\rho}\right)\left[1-2 \delta+\left(\rho+\ell_{\rho}\right) \ell_{\rho} \delta^{2}-\rho \ell_{\rho}^{3} \delta^{2}\right]}{\left(1-\rho^{2}\right)\left(1-\rho \ell_{\rho}\right)\left(1-\ell_{\rho}^{2} \delta\right)^{2}\left(1-\rho \ell_{\rho} \delta\right)^{2}}-\frac{\ell_{\rho}^{3}(1-\delta)\left[1-2 \delta+\ell_{\rho}^{2}(1+\delta) \delta^{2}-\ell_{\rho}^{4} \delta^{3}\right]}{\left(1-\ell_{\rho}^{2} \delta\right)^{3}\left(1-\ell_{\rho}^{2} \delta^{2}\right)^{2}} .
$$

We next substitute $\ell_{s}$ from (A.45) into the term $\frac{\ell_{s}}{1-\ell_{\rho}^{2}}$ that multiplies the first square bracket in (A.46). Grouping terms, we can write (A.46) as

$$
-\frac{\nu \rho\left(\rho-\ell_{\rho}\right)}{\left(1-\rho^{2}\right)\left(1-\rho \ell_{\rho}\right)^{2}} H_{1}\left(\ell_{\rho}\right)+\frac{\ell_{\rho}^{2}(1-\delta)}{\left(1-\ell_{\rho}^{2} \delta\right)^{2}} H_{2}\left(\ell_{\rho}\right)=0
$$

Eq. (A.47) coincides with (27) when $\ell_{\rho}=r$. To show that (A.47) has a solution, we note 
that $H_{1}\left(\ell_{\rho}\right), H_{2}\left(\ell_{\rho}\right)>0$ for $\ell_{\rho} \in[0,1]$ because

$$
\begin{aligned}
& 2-\rho \ell_{\rho}(1+\delta)-\ell_{\rho}^{2} \delta+\rho^{2} \ell_{\rho}^{4} \delta^{2}>0 \\
& 2-\ell_{\rho}^{2} \delta^{2}-\ell_{\rho}^{4} \delta^{3}>0 .
\end{aligned}
$$

(To show (A.48) and (A.49), we use $\rho, \delta<1$. For (A.48) we also note that the left-hand side (LHS) is decreasing in $\delta$ and positive for $\delta=1$.) Since $H_{1}\left(\ell_{\rho}\right), H_{2}\left(\ell_{\rho}\right)>0$, the LHS of (A.47) is negative for $\ell_{\rho}=0$ and positive for $\ell_{\rho}=\rho$. Therefore, there exists a solution $\ell_{\rho}=r$ in $(0, \rho)$. Moreover, there does not exist a solution in $(\rho, 1]$ because the LHS is positive. Substituting $\ell_{\rho}=r$ into (A.45), we find $\ell_{s}=z$. The argument that $\ell_{\rho} \neq\{0,1\}$ is along the same lines as in the proof of Proposition 5.

Proof of Proposition 8: Proceeding as in the proof of Proposition 6, we find

$$
\sum_{k^{\prime}=1}^{k} \frac{\partial \tilde{E}_{t-1}\left(s_{t}\right)}{\partial s_{t-k^{\prime}}}-\sum_{k^{\prime}=1}^{k} \frac{\partial E_{t-1}\left(s_{t}\right)}{\partial s_{t-k^{\prime}}}=\alpha g_{k}+o(\alpha)
$$

where

$$
\begin{aligned}
g_{k} & \equiv \sum_{k^{\prime}=1}^{k} f_{k^{\prime}} \\
f_{k} & \equiv r^{k}\left(\frac{z}{1-r^{2}}-\delta^{k-1}\right)-\rho^{k} \frac{\nu}{1-\rho^{2}}
\end{aligned}
$$

The proposition will follow if we show that $f_{1}=g_{1}<0$ and $g_{\infty}<0$. Indeed, suppose that $f_{1}=g_{1}<0$. Since $r<\rho, f_{k}$ is negative for large $k$. Moreover, (A.43) can be written for $\left(\ell_{s}, \ell_{\rho}\right)=(z, r)$ as

$$
\sum_{k=1}^{\infty} r^{k} f_{k}=0
$$

implying that $f_{k}$ has to be positive for some $k$. Since $f_{k}$ can change sign at most twice (because the derivative of $f_{k} / \rho^{k}$ can change sign at most once, implying that $f_{k} / \rho^{k}$ can change sign at most twice), it is negative, then positive, and then negative again.

The function $g_{k}$ is negative for $k=1$, then decreases $\left(f_{k}<0\right)$, then increases $\left(f_{k}>0\right)$, and then decreases again $\left(f_{k}<0\right)$. If $g_{\infty}<0$, then $g_{k}$ can either be (i) always negative or 
(ii) negative, then positive, and then negative again. To rule out (i), we write (A.51) as

$$
g_{1} r+\sum_{k=2}^{\infty}\left(g_{k}-g_{k-1}\right) r^{k}=0 \Leftrightarrow \sum_{k=1}^{\infty} g_{k}\left(r^{k}-r^{k+1}\right)=0 .
$$

We next show that $f_{1}=g_{1}<0$ and $g_{\infty}<0$. Substituting $z$ from (26), we find

$$
\begin{aligned}
f_{1}=g_{1} & =\frac{z r}{1-r^{2}}-r-\frac{\nu \rho}{1-\rho^{2}}=\frac{\rho r(\rho-r)\left(\nu-\nu_{1}\right)}{\left(1-\rho^{2}\right)(1-\rho r)}, \\
g_{\infty} & =\frac{z r}{\left(1-r^{2}\right)(1-r)}-\frac{r}{1-r \delta}-\frac{\nu \rho}{\left(1-\rho^{2}\right)(1-\rho)}=\frac{\rho(\rho-r)\left(\nu_{\infty}-\nu\right)}{\left(1-\rho^{2}\right)(1-\rho r)(1-\rho)},
\end{aligned}
$$

where

$$
\begin{aligned}
\nu_{1} & \equiv \frac{\left(1-\rho^{2}\right)(1-\rho r) r^{2}(1-\delta)}{\rho(\rho-r)\left(1-r^{2} \delta\right)} \\
\nu_{\infty} & \equiv \frac{\left(1-\rho^{2}\right)(1-\rho r)(1-\rho) r^{2}(1-\delta)}{\rho(\rho-r)\left(1-r^{2} \delta\right)(1-r \delta)} .
\end{aligned}
$$

We thus need to show that $\nu_{1}>\nu>\nu_{\infty}$. These inequalities will follow if we show that when $\nu$ is replaced by $\nu_{1}$ (resp. $\nu_{\infty}$ ) in (27), the LHS becomes larger (resp. smaller) than the RHS. To show these inequalities, we make use of $0<r<\rho<1$ and $0 \leq \delta<1$. The inequality for $\nu_{1}$ is

$$
\frac{(\rho-r \delta) r^{2}}{(\rho-r)(1-\rho r)\left(1-r^{2} \delta\right)}+\frac{Y_{1}}{(1-\rho r)(1-\rho r \delta)^{2}\left(1-r^{2} \delta^{2}\right)^{2}\left(1-r^{2} \delta\right)}>0
$$

where

$$
Y_{1}=\left(1-r^{2} \delta^{2}\right)^{2}\left[2-\rho r(1+\delta)-r^{2} \delta+\rho^{2} r^{4} \delta^{2}\right]-(1-\rho r)(1-\rho r \delta)^{2}\left(2-r^{2} \delta^{2}-r^{4} \delta^{3}\right)
$$

Since $\rho>r>r \delta,(\mathrm{A} .52)$ holds if $Y_{1}>0$. Algebraic manipulations show that $Y_{1}=(\rho-r \delta) r Z_{1}$, where

$$
\begin{aligned}
Z_{1} \equiv & \left(2-r^{2} \delta^{2}-r^{4} \delta^{3}\right)\left[\delta\left(1-r^{2} \delta\right)\left(2-r^{2} \delta^{2}-\rho r \delta\right)+(1-\rho r \delta)^{2}\right] \\
& -\left(1-r^{2} \delta^{2}\right)^{2}\left[1+\delta-(\rho+r \delta) r^{3} \delta^{2}\right]
\end{aligned}
$$


Since $2-r^{2} \delta^{2}-r^{4} \delta^{3}>2\left(1-r^{2} \delta^{2}\right)$, inequality $Z_{1}>0$ follows from

$$
2\left[\delta\left(1-r^{2} \delta\right)\left(2-r^{2} \delta^{2}-\rho r \delta\right)+(1-\rho r \delta)^{2}\right]-\left(1-r^{2} \delta^{2}\right)\left[1+\delta-(\rho+r \delta) r^{3} \delta^{2}\right]>0
$$

To show (A.53), we break the LHS into

$$
2 \delta\left(1-r^{2} \delta\right)\left(1-r^{2} \delta^{2}\right)-\left(1-r^{2} \delta^{2}\right)\left(\delta-r^{4} \delta^{3}\right)=\delta\left(1-r^{2} \delta^{2}\right)\left(1-r^{2} \delta\right)^{2}>0
$$

and

$$
2\left[\delta\left(1-r^{2} \delta\right)(1-\rho r \delta)+(1-\rho r \delta)^{2}\right]-\left(1-r^{2} \delta^{2}\right)\left(1-\rho r^{3} \delta^{2}\right)
$$

Eq. (A.54) is positive because of the inequalities

$$
\begin{aligned}
& 2(1-\rho r \delta)>1-r^{2} \delta^{2}, \\
& \delta\left(1-r^{2} \delta\right)+1-\rho r \delta>1-\rho r^{3} \delta^{2} .
\end{aligned}
$$

The inequality for $\nu_{\infty}$ is

$$
-\frac{(\rho-r \delta)(1-\rho)(1-r) r}{(\rho-r)(1-\rho r)\left(1-r^{2} \delta\right)(1-r \delta)^{2}}+\frac{Y_{\infty}}{(1-\rho r)(1-\rho r \delta)^{2}\left(1-r^{2} \delta\right)\left(1-r^{2} \delta^{2}\right)^{2}(1-r \delta)}<0
$$

where

$Y_{\infty}=(1-\rho)\left(1-r^{2} \delta^{2}\right)\left[2-\rho r(1+\delta)-r^{2} \delta+\rho^{2} r^{4} \delta^{2}\right]-(1-\rho r)(1-\rho r \delta)^{2}(1-r \delta)\left(2-r^{2} \delta^{2}-r^{4} \delta^{3}\right)$

Algebraic manipulations show that $Y_{\infty}=-(\rho-r \delta) Z_{\infty}$, where

$$
\begin{aligned}
Z_{\infty} \equiv & \left(2-r^{2} \delta^{2}-r^{4} \delta^{3}\right)\left[(1-\rho r \delta)^{2}(1-r)-(1-\rho) r \delta\left(1-r^{2} \delta\right)\left(2-r^{2} \delta^{2}-\rho r \delta\right)\right] \\
& +(1-\rho) r\left(1-r^{2} \delta^{2}\right)^{2}\left[1+\delta-(\rho+r \delta) r^{3} \delta^{2}\right] .
\end{aligned}
$$

To show that $Z_{\infty}>0$, we break it into

$$
\begin{aligned}
& \left(2-r^{2} \delta^{2}-r^{4} \delta^{3}\right)\left[(1-\rho r \delta)^{2}(1-r)-(1-\rho) r \delta\left(1-r^{2} \delta\right)(1-\rho r \delta)\right] \\
> & \left(2-r^{2} \delta^{2}-r^{4} \delta^{3}\right)(1-\rho r \delta)\left[(1-\rho r \delta)(1-r)-(1-\rho)\left(1-r^{2} \delta\right)\right] \\
= & \left(2-r^{2} \delta^{2}-r^{4} \delta^{3}\right)(1-\rho r \delta)(\rho-r)(1-r \delta)>0
\end{aligned}
$$


and

$$
\begin{aligned}
& (1-\rho) r\left(1-r^{2} \delta^{2}\right)^{2}\left[1+\delta-(\rho+r \delta) r^{3} \delta^{2}\right]-\left(2-r^{2} \delta^{2}-r^{4} \delta^{3}\right)(1-\rho) r \delta\left(1-r^{2} \delta\right)\left(1-r^{2} \delta^{2}\right) \\
= & (1-\rho) r\left(1-r^{2} \delta^{2}\right)\left[\left(1-r^{2} \delta^{2}\right)\left[1+\delta-(\rho+r \delta) r^{3} \delta^{2}\right]-\left(2-r^{2} \delta^{2}-r^{4} \delta^{3}\right) \delta\left(1-r^{2} \delta\right)\right] .
\end{aligned}
$$

Since $2-r^{2} \delta^{2}-r^{4} \delta^{3}<2-r^{2} \delta^{2}-r^{4} \delta^{4}=\left(1-r^{2} \delta^{2}\right)\left(2+r^{2} \delta^{2}\right)$, the last square bracket is greater than

$$
\begin{aligned}
& \left(1-r^{2} \delta^{2}\right)\left[1+\delta-(\rho+r \delta) r^{3} \delta^{2}-\delta\left(1-r^{2} \delta\right)\left(2+r^{2} \delta^{2}\right)\right] \\
= & \left(1-r^{2} \delta^{2}\right)\left[(1-\delta)\left(1-r^{4} \delta^{3}\right)+r^{2} \delta^{2}(2-\delta-\rho r)\right]>0 .
\end{aligned}
$$

Proof of Proposition 9: Eqs. (A.6) and (A.7) imply that in steady state

$$
\tilde{E}_{t-1}\left(s_{t}\right) \equiv \bar{s}_{t}(\tilde{p})=\sum_{k=1}^{\infty} \tilde{N}_{k} \zeta_{t-k}+\tilde{\mu}+\sum_{k=1}^{\infty} \tilde{C} \tilde{D}^{k-1} \tilde{G}(\mu-\tilde{\mu})
$$

Therefore,

$$
\tilde{E}_{t}\left(s_{t+1}\right)-\tilde{E}_{t-1}\left(s_{t}\right)=\sum_{k=1}^{\infty}\left(\tilde{N}_{k}-\tilde{N}_{k-1}\right) \zeta_{t-k}
$$

where $\tilde{N}_{0} \equiv 0$. Since the sequence $\left\{\zeta_{t}\right\}_{t \in \mathbb{Z}}$ is i.i.d. and normal with mean zero and variance $\sigma_{s}^{2}$, the variable $\tilde{E}_{t}\left(s_{t+1}\right)-\tilde{E}_{t-1}\left(s_{t}\right)$ is normal with mean zero and variance $\sigma_{s}^{2} \sum_{k=1}^{\infty}\left(\tilde{N}_{k}-\tilde{N}_{k-1}\right)^{2}$. Eq. (31) then follows from (30) and the fact that for a normal variable $\gamma$ with mean zero and variance $\sigma^{2}, E(|\gamma|)=\sqrt{\frac{2}{\pi}} \sigma$.

Suppose that $\sigma_{\eta}^{2}=0$ and Freddy has prior knowledge that $\tilde{\rho} \geq \underline{\rho}$ for $\underline{\rho} \in(0, \rho]$. Since $N_{k}=0$ for all $k \geq 1,(31)$ implies that $Q=0$. To show that $\tilde{Q}>0$, suppose instead that $\tilde{Q}=0$. Eq. (31) then implies that $\tilde{N}_{k}=0$ for all $k \geq 1$, Lemma A.2 implies that $\tilde{C} \tilde{A}^{k-1} \tilde{G}=0$ for all $k \geq 1$, and Proposition 4 implies that $\tilde{\rho}=0$, a contradiction.

Suppose next that $\rho>0$ and Freddy has no prior knowledge. Eq. (A.42) implies that

$$
\tilde{N}_{k}-\tilde{N}_{k-1}=\alpha h_{k}+o(\alpha)
$$


where

$$
\tilde{h}_{k} \equiv r^{k}\left(\frac{z}{1-r^{2}}-\delta^{k-1}\right)-r^{k-1}\left(\frac{z}{1-r^{2}}-\delta^{k-2}\right)=-r^{k-1}\left[\frac{z(1-r)}{1-r^{2}}-\delta^{k-2}(1-r \delta)\right]
$$

for $k \geq 2$, and

$$
\tilde{h}_{1} \equiv r\left(\frac{z}{1-r^{2}}-1\right)
$$

Substituting into (31), we find

$$
\tilde{Q}=\sqrt{\frac{2}{\pi}} \frac{\alpha}{a \sigma_{s}} \sqrt{\sum_{k=1}^{\infty} \tilde{h}_{k}^{2}}+o(\alpha)
$$

The same equation holds for Tommy by omitting the tildes and setting

$$
h_{k} \equiv \rho^{k} \frac{\nu}{1-\rho^{2}}-\rho^{k-1} \frac{\nu}{1-\rho^{2}}=-\rho^{k-1} \frac{\nu}{1+\rho}
$$

for $k \geq 2$, and

$$
h_{1} \equiv \rho \frac{\nu}{1-\rho^{2}}
$$

Therefore, for small $\alpha$ and $\sigma_{\eta}^{2}$, inequality $\tilde{Q}>Q$ is equivalent to

$$
\begin{aligned}
& \sum_{k=1}^{\infty} \tilde{h}_{k}^{2}>\sum_{k=1}^{\infty} h_{k}^{2} \\
\Leftrightarrow & \frac{z^{2} r^{2}}{\left(1-r^{2}\right)^{2}(1+r)}+\frac{r^{2}}{1+r \delta}-\frac{z r^{2}(2-r-r \delta)}{\left(1-r^{2}\right)\left(1-r^{2} \delta\right)}>\frac{\nu^{2} \rho^{2}}{\left(1-\rho^{2}\right)^{2}(1+\rho)} .
\end{aligned}
$$

Substituting $z$ from (26) and grouping terms in $\nu^{2}, \nu$, and 1 , we can write (A.56) as $R_{Q}>0$ where

$$
R_{Q} \equiv \frac{\nu^{2} \rho^{2}(\rho-r)\left(1+r-r^{2}-\rho r^{2}\right)}{\left(1-\rho^{2}\right)^{2}(1-\rho r)^{2}(1+\rho)}-\frac{\nu \rho r^{2}\left(1-r^{2}\right)(1-\delta)}{\left(1-\rho^{2}\right)(1-\rho r)\left(1-r^{2} \delta\right)}+\frac{r^{4}(1-\delta)^{2}}{\left(1-r^{2} \delta\right)^{2}(1+r \delta)}
$$

When $\nu$ is small, (27) implies that

$$
r \approx \sqrt{\frac{\nu \rho^{2}}{\left(1-\rho^{2}\right)(1-\delta)}}
$$


Substituting into (A.57), we find

$$
R_{Q} \approx \frac{\nu^{2} \rho^{5}}{\left(1-\rho^{2}\right)^{2}(1+\rho)}>0
$$

When $\nu$ is large, $(27)$ implies that $r \approx \rho$ and

$$
\rho-r \approx \frac{\rho\left(1-\rho^{2}\right)^{3}(1-\delta)}{\nu\left(1-\rho^{2} \delta\right)^{2}}
$$

Substituting into (A.57), we find

$$
R_{Q} \approx-\frac{\nu \rho^{5}(1-\delta)^{2}}{\left(1-\rho^{2}\right)\left(1-\rho^{2} \delta\right)^{2}}<0
$$

Therefore, $\tilde{Q}>Q$ for small $\nu$, and $\tilde{Q}<Q$ for large $\nu$.

Proof of Proposition 10: The maximum in (28) is

$$
\tilde{U}_{1}\left(\tilde{W}_{t-1}, \tilde{E}_{t-1}\left(s_{t}\right)\right)=-\exp \left[-a(1+r) \tilde{W}_{t-1}-\frac{\left(\tilde{E}_{t-1}\left(s_{t}\right)-r\right)^{2}}{2 \tilde{V} a r_{1}}\right]
$$

and the maximum when Freddy observes signals up to Period $t-k$ only is

$$
\tilde{U}_{k}\left(\tilde{W}_{t-1}, \tilde{E}_{t-k}\left(s_{t}\right)\right)=-\exp \left[-a(1+r) \tilde{W}_{t-1}-\frac{\left(\tilde{E}_{t-k}\left(s_{t}\right)-r\right)^{2}}{2 \tilde{V} a r_{k}}\right]
$$

Eqs. (32), (A.60), and (A.61) imply that

$$
\exp \left[a(1+r) \tilde{I}_{k}\right] \tilde{E}_{t-k-1}\left\{\exp \left[-\frac{\left(\tilde{E}_{t-1}\left(s_{t}\right)-r\right)^{2}}{2 \tilde{V} a r_{1}}\right]\right\}=\exp \left[-\frac{\left(\tilde{E}_{t-k-1}\left(s_{t}\right)-r\right)^{2}}{2 \tilde{V} a r_{k+1}}\right] .
$$

To compute the expectation in (A.62), we write the term inside the square bracket as $-\frac{\gamma^{2}}{2}$, where

$$
\gamma \equiv \frac{\tilde{E}_{t-1}\left(s_{t}\right)-r}{\sqrt{\tilde{V} a r_{1}}} .
$$

When Freddy observes signals up to Period $t-k-1$, he takes $\gamma$ to be normal with mean

$$
\mu_{\gamma} \equiv \frac{\tilde{E}_{t-k-1}\left(s_{t}\right)-r}{\sqrt{\tilde{V} a r_{1}}}
$$


and variance

$$
\sigma^{2} \equiv \frac{\tilde{V} a r_{t-k-1}\left(\tilde{E}_{t-1}\left(s_{t}\right)\right)}{\tilde{V} a r_{1}}=\frac{\tilde{V} a r_{t-k-1}\left(s_{t}\right)-\tilde{E}_{t-k-1}\left(\tilde{V} a r_{t-1}\left(s_{t}\right)\right)}{\tilde{V} a r_{1}}=\frac{\tilde{V} a r_{k+1}-\tilde{V} a r_{1}}{\tilde{V} a r_{1}}
$$

Since for a normal variable $\gamma$ with mean $\mu_{\gamma}$ and variance $\sigma^{2}$,

$$
E \exp \left(-\frac{\gamma^{2}}{2}\right)=\frac{1}{\sqrt{1+\sigma^{2}}} \exp \left(-\frac{\mu_{\gamma}^{2}}{2\left(1+\sigma^{2}\right)}\right)
$$

we have

$$
\tilde{E}_{t-k-1}\left\{\exp \left[-\frac{\left(\tilde{E}_{t-1}\left(s_{t}\right)-r\right)^{2}}{2 \tilde{V} a r_{1}}\right]\right\}=\sqrt{\frac{\tilde{V} a r_{1}}{\tilde{V} a r_{k+1}}} \exp \left[-\frac{\left(\tilde{E}_{t-k-1}\left(s_{t}\right)-r\right)^{2}}{2 \tilde{V} a r_{k+1}}\right]
$$

Substituting into (A.62), we find the first equality in (33). To derive the second equality, we note from (A.63) that

$$
\tilde{\operatorname{Var}}{ }_{k+1}=\tilde{V} a r_{1}+\tilde{\operatorname{Var}} r_{t-k-1}\left(\tilde{E}_{t-1}\left(s_{t}\right)\right)
$$

We next define the sequence $\left\{\tilde{\zeta}_{t}\right\}_{t \in \mathbb{Z}}$ of surprises according to Freddy by $\tilde{\zeta}_{t} \equiv s_{t}-\tilde{E}_{t-1}\left(s_{t}\right)$. Same calculations as for Tommy in Lemma A.1 imply that in steady state

$$
\tilde{E}_{t-1}\left(s_{t}\right)=\sum_{k=1}^{\infty} \tilde{C} \tilde{A}^{k-1} \tilde{G} \tilde{\zeta}_{t-k}
$$

Therefore,

$$
\tilde{V} a r_{t-k-1}\left(\tilde{E}_{t-1}\left(s_{t}\right)\right)=\tilde{V} a r_{t-k-1}\left(\sum_{k^{\prime}=1}^{k} \tilde{C} \tilde{A}^{k^{\prime}-1} \tilde{G} \tilde{\zeta}_{t-k^{\prime}}\right)=\sum_{k^{\prime}=1}^{k}\left(\tilde{C} \tilde{A}^{k-1} \tilde{G}\right)^{2} \tilde{V} a r_{1}
$$

where the last step is because Freddy views the sequence $\left\{\tilde{\zeta}_{t}\right\}_{t \in \mathbb{Z}}$ as i.i.d. with variance $\tilde{V} a r_{1}=\sigma_{s}^{2}(\tilde{p})$. Combining (A.64) and (A.65) establishes the second inequality in (33).

Suppose that $\sigma_{\eta}^{2}=0$ and Freddy has prior knowledge that $\tilde{\rho} \geq \underline{\rho}$ for $\underline{\rho} \in(0, \rho]$. Since $N_{k}=0$ for all $k \geq 1$, (33) implies that $I_{k}=0$ for all $k \geq 1$. To show that $\tilde{I}_{k}>0$ for all $k \geq 2$, it suffices to show that $\tilde{I}_{2}>0$. If $\tilde{I}_{2}=0$, then $\tilde{C} \tilde{G}=0$ and $\tilde{C} \tilde{A} \tilde{G}=0$. Proceeding as in the proof of Proposition 4 , we can show that $\tilde{\rho}=0$, a contradiction.

Suppose next that $\rho>0$ and Freddy has no prior knowledge. Eqs. (33) and (A.42) imply 
that

$$
\tilde{I}_{k}=\frac{\alpha^{2}}{2 a(1+r)} \sum_{k^{\prime}=1}^{k} r^{2 k^{\prime}}\left(\frac{z}{1-r^{2}}-\delta^{k^{\prime}-1}\right)^{2}+o\left(\alpha^{2}\right)
$$

Likewise,

$$
I_{k}=\frac{\alpha^{2}}{2 a(1+r)} \sum_{k^{\prime}=1}^{k} \rho^{2 k^{\prime}} \frac{\nu^{2}}{\left(1-\rho^{2}\right)^{2}}+o\left(\alpha^{2}\right) .
$$

Therefore, for small $\alpha$ and $\sigma_{\eta}^{2}$,

$$
\tilde{I}_{k}>I_{k} \Leftrightarrow \sum_{k^{\prime}=1}^{k}\left[r^{k^{\prime}}\left(\frac{z}{1-r^{2}}-\delta^{k^{\prime}-1}\right)+\rho^{k^{\prime}} \frac{\nu}{1-\rho^{2}}\right] f_{k^{\prime}}>0
$$

for $f_{k}$ defined in (A.50). Recall from Proposition 8 that $f_{k}$ is negative in an interval $k \in$ $\left\{1, . ., k_{0}-1\right\}$, becomes positive in an interval $k \in\left\{k_{0}, . ., k_{1}\right\}$, and becomes negative again for $k \in\left\{k_{1}+1, ..\right\}$. Since $f_{k}<0$ in $\left\{k_{1}+1, ..\right\}$, (A.51) implies that

$$
\sum_{k=1}^{k_{1}} r^{k} f_{k}=-\sum_{k=k_{1}+1}^{\infty} r^{k} f_{k}>0
$$

Since $f_{k}<0$ in $\left\{1, . ., k_{0}\right\}, f_{k}>0$ in $k \in\left\{k_{0}+1, . ., k_{1}\right\}$, and $0<r<\rho$,

$$
\sum_{k=1}^{k_{1}} \rho^{k} f_{k}=\rho^{k_{0}} \sum_{k=1}^{k_{1}} \rho^{k-k_{0}} f_{k}>\rho^{k_{0}} \sum_{k=1}^{k_{1}} r^{k-k_{0}} f_{k}=\frac{\rho^{k_{0}}}{r^{k_{0}}} \sum_{k=1}^{k_{1}} r^{k} f_{k} .
$$

Likewise, since $0 \leq \delta<1$,

$$
\sum_{k=1}^{k_{1}} r^{k} \delta^{k-1} f_{k}=\delta^{k_{0}-1} \sum_{k=1}^{k_{1}} r^{k} \delta^{k-k_{0}} f_{k}<\delta^{k_{0}-1} \sum_{k=1}^{k_{1}} r^{k} f_{k}
$$

Eqs (A.68), (A.70), and (A.71) imply that $\tilde{I}_{k_{1}}>I_{k_{1}}$ if

$$
\frac{1}{r^{k_{0}}}\left[r^{k_{0}}\left(\frac{z}{1-r^{2}}-\delta^{k_{0}-1}\right)+\rho^{k_{0}} \frac{\nu}{1-\rho^{2}}\right]\left(\sum_{k=1}^{k_{1}} r^{k} f_{k}\right)>0 .
$$

This inequality holds because of (A.68) and

$$
r^{k_{0}}\left(\frac{z}{1-r^{2}}-\delta^{k_{0}-1}\right)+\rho^{k_{0}} \frac{\nu}{1-\rho^{2}}=f_{k_{0}}+2 \rho^{k_{0}} \frac{\nu}{1-\rho^{2}}>0
$$


Computing the sums in (A.66) and (A.67), we find that $\tilde{I}_{\infty}>I_{\infty}$ is equivalent to

$$
\frac{z^{2} r^{2}}{\left(1-r^{2}\right)^{3}}+\frac{r^{2}}{1-r^{2} \delta^{2}}-\frac{2 z r^{2}}{\left(1-r^{2}\right)\left(1-r^{2} \delta\right)}>\frac{\nu^{2} \rho^{2}}{\left(1-\rho^{2}\right)^{3}} .
$$

Substituting $z$ from (26) and grouping terms in $\nu^{2}, \nu$, and 1 , we can write (A.72) as $R_{I}>0$ where

$$
R_{I} \equiv-\frac{\nu^{2} \rho^{2}(\rho-r)^{2}}{\left(1-\rho^{2}\right)^{3}(1-\rho r)^{2}}+\frac{r^{4}(1-\delta)^{2}}{\left(1-r^{2} \delta^{2}\right)\left(1-r^{2} \delta\right)^{2}} .
$$

To evaluate $R_{I}$ for small $\nu$, we substitute (A.58) into (A.73) and find

$$
R_{I} \approx-\frac{\nu^{2} \rho^{6}}{\left(1-\rho^{2}\right)^{3}}<0
$$

To evaluate $R_{I}$ for large $\nu$, we substitute (A.59) into (A.73) and find

$$
R_{I} \approx \frac{\rho^{6}(1-\delta)^{4}}{\left(1-\rho^{2} \delta\right)^{4}\left(1-\rho^{2} \delta^{2}\right)}>0
$$

Therefore, $\tilde{I}_{\infty}<I_{\infty}$ for small $\nu$, and $\tilde{I}_{\infty}>I_{\infty}$ for large $\nu$. 


\section{References}

Anderson, B. and J. Moore, 1979, Optimal Filtering, Prentice Hall, New York.

Balakrishnan, A., 1987, Kalman Filtering Theory, Optimization Software Inc., Publications Division, New York.

Baquero, G. and M. Verbeek, 2006, "Do Sophisticated Investors Believe in the Law of Small Numbers?" working paper, Rotterdam School of Management.

Bar-Hillel, M. and W. Wagenaar, 1991, "Perceptions of Randomness," Advances of Applied Mathematics, 12, 428-454.

Barberis, N., A. Shleifer and R. Vishny, 1998, "A Model of Investor Sentiment," Journal of Financial Economics, 49, 307-343.

Berk, J. and R. Green, 2004, "Mutual Fund Flows and Performance in Rational Markets," Journal of Political Economy, 112, 1269-1295.

Billingsley, P., 1986, Probability and Measure, John Wiley, New York.

Budescu, D. and A. Rapoport, 1994, "Subjective Randomization in One- and TwoPerson Games," Journal of Behavioral Decision Making, 7, 261-278.

Camerer, C., 1989, "Does the Basketball Market Believe in the 'Hot Hand'?" American Economic Review, 79, 1257-1261.

Carhart, M., 1997, "On Persistence in Mutual Fund Performance," Journal of Finance, $52,57-82$.

Chevalier, J. and G. Ellison, 1997, "Risk Taking by Mutual Funds as a Response to Incentives," Journal of Political Economy, 105, 1167-1200.

Clotfelter, C. and P. Cook, 1993, "The 'Gambler's Fallacy in Lottery Play," Management Science, 39, 1521-1525.

Edwards, W., 1961, "Probability Learning in 1000 trials," Journal of Experimental Psychology, 62, 385-394.

Fama, E., 1991, "Efficient Capital Markets: II," Journal of Finance, 46, 1575-1617. 
Gilovich, T., R. Vallone and A. Tversky, 1985, "The Hot Hand in Basketball: On the Misperception of Random Sequences," Cognitive Psychology, 17, 295-314.

Jagannathan, R., A. Malakhov and D. Novikov, 2006, "Do Hot Hands Persist Among Hedge Fund Managers? An Empirical Evaluation," NBER working paper 12015.

McLeish, D., 1975, "A Maximal Inequality and Dependent Strong Laws," The Annals of Probability, 3, 829-839.

Mehra, R. and E. Prescott, 1985, "The Equity Premium: A Puzzle," Journal of Monetary Economics, 15, 145-161.

Merton, R., 1971, "Optimum Consumption and Portfolio Rules in a Continuous-Time Model," Journal of Economic Theory, 3, 373-413.

Rabin, M., 2002, "Inference by Believers in the Law of Small Numbers," Quarterly Journal of Economics, 117, 775-816.

Rabin, M. and D. Vayanos, 2005, "The Gambler's and Hot-Hand Fallacy in a DynamicInference Model," working paper, LSE and UC Berkeley.

Rapoport, A. and D. Budescu, 1992, "Generation of Random Binary Series in Strictly Competitive Games," Journal of Experimental Psychology: General, 121, 352-364.

Rapoport, A. and D. Budescu, 1997, "Randomization in Individual Choice Behavior," Psychological Review, 104, 603-617.

Terrell, D., 1994, "A Test of the Gambler's Fallacy - Evidence from Pari-Mutuel Games," Journal of Risk and Uncertainty, 8, 309-317.

Tversky, A. and T. Gilovich, 1989a, "The Cold Facts about the 'Hot Hand' in Basketball," Chance, 2, 16-21.

Tversky, A. and T. Gilovich, 1989b, "The Hot Hand: Statistical Reality or Cognitive Illusion?" Chance, 2, 31-34.

Sirri, E. and P. Tufano, 1998, "Costly Search and Mutual Fund Flows," Journal of Finance, 53, 1655-1694. 